\author{
A Thesis by \\ Armin Ghoddoussi \\ Bachelor of Science, Sojo University, 1998 \\ Submitted to Department of Aerospace Engineering \\ and the faculty of the Graduate School of \\ Wichita State University \\ in partial fulfillment of \\ the requirements for the degree of \\ Master of Science
}

July 2011 
(C) Copyright 2011 by Armin Ghoddoussi

All Rights Reserved 


\section{A CONCEPTUAL STUDY OF AIRFOIL PERFORMANCE ENHANCEMENTS USING CFD}

The following faculty members have examined the final copy of this thesis for form and content, and recommend that it be accepted in partial fulfillment of the requirement for the degree of Masters of Science with a major in Aerospace Engineering.

L. Scott Miller, Committee Chair

Klaus Hoffmann, Committee Member

Krishna Krishnan, Committee Member 


\section{DEDICATION}

To my beloved parents, my brother and my dearest friends 
Anyone who has never made a mistake, has never tried anything new. 


\section{ACKNOWLEDGEMENTS}

I would like to thank my adviser, Dr. Scott Miller, for his many years of thoughtful, patient guidance and support. Thanks are also due to the members of my committee, Klaus Hoffmann and

Krishna Krishnan, for their helpful comments and suggestions on all stages of this project. I also want to thank my friends and colleagues, Mohammad A. Badr, Johann Dorfling and Joey Lemon for all their help, opinions and collaborations. 


\begin{abstract}
A conceptual study of performance enhancing devices for an airfoil is performed using Computational Fluid Dynamics. Three simple, passive devices are examined to explore alternate methods for stall control and lift-to-drag improvement. The motivation behind this research is to study effective techniques to improve performance with fewer drawbacks than previously existing methods. An evaluation scheme is presented to compute airfoil lift, drag and pitching moment for a range of angles-of-attack up to stall. NACA $64_{1}-212$ single-element and slatted airfoil CFD results are compared with experimental data to validate the computational model. Evaluations on the first conceptual design (Stall vane) show elimination of the separation at 15 degrees of angle-of-attack where the flow reversal normally starts at $86 \%$ - chord. A total drag increase of $22 \%$ is detected because of the sharp leading-edge of the device, but the main element drag has a reduction of $43 \%$. The maximum lift coefficient does not show a significant change on the same model. The second device (Cylinder) has a negative effect, initiating flow separation and causing a significant decrease in lift-to-drag ratio at a given lift coefficient. The third device (Dimples) demonstrates the potential of lift-to-drag ratio improvement at the higher angle-of-attack. Further investigation is required to verify the results since the improvement is small.
\end{abstract}




\section{TABLE OF CONTENTS}

Chapter

Pages

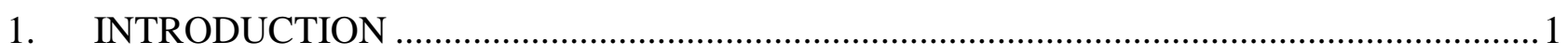

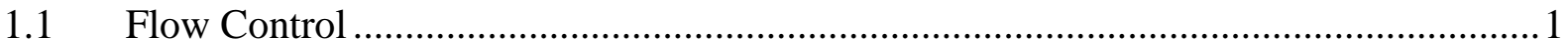

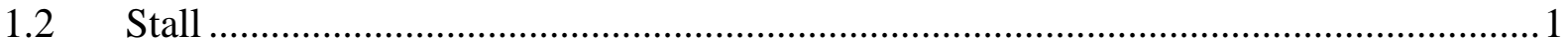

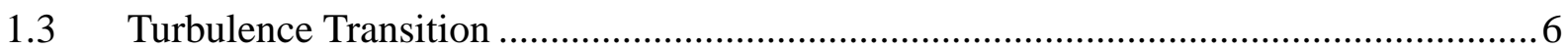

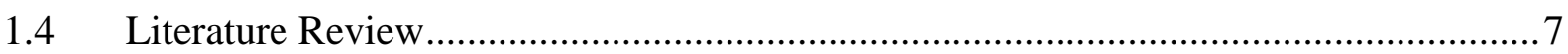

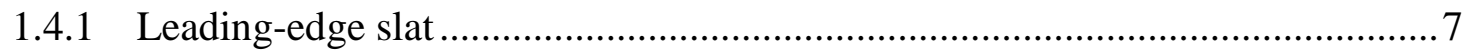

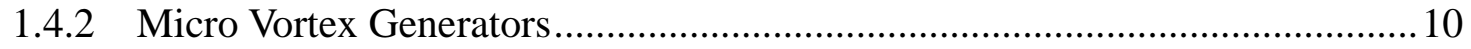

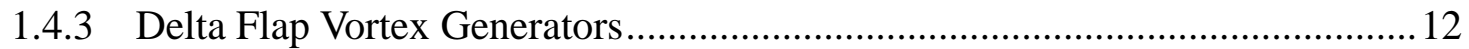

1.4.4 Turbulence Manipulator and Large Eddy Breakup Device ............................ 13

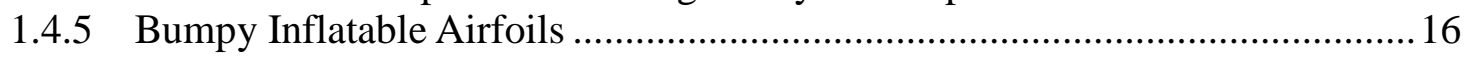

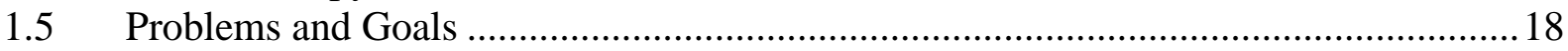

2. NEW DESIGN CONCEPTS AND SCREENING ....................................................20

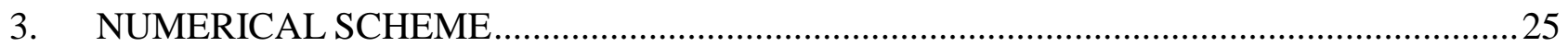

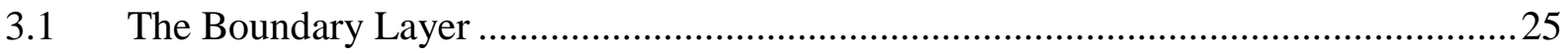

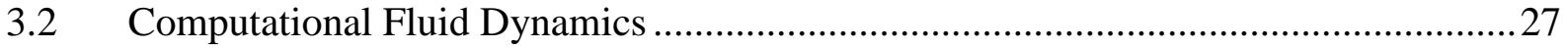

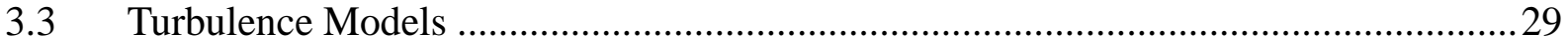

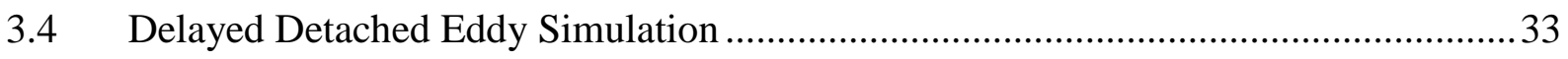

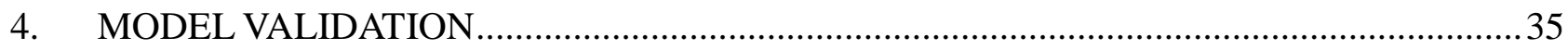

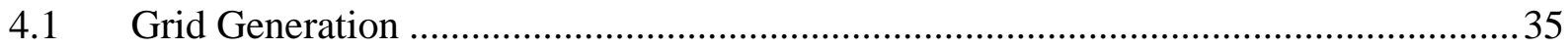

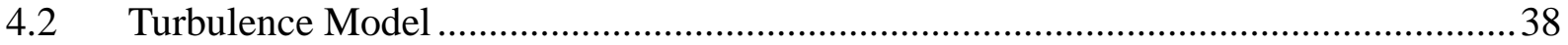

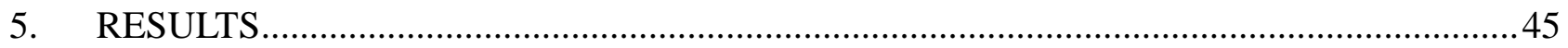

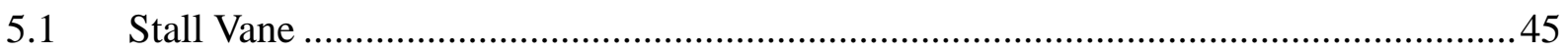

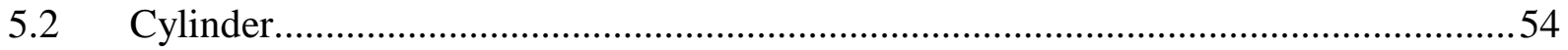

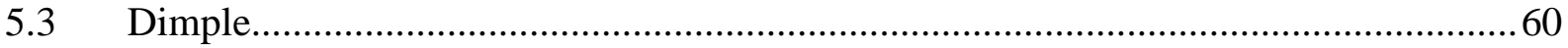

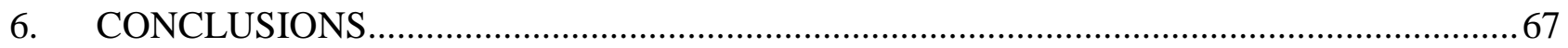

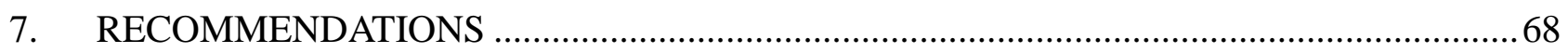

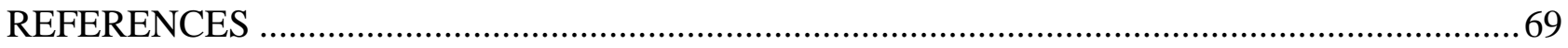




\section{LIST OF TABLES}

Table

Page

1. Summary of Maximum Lift Characteristics and Leading-edge Slat Configuration for

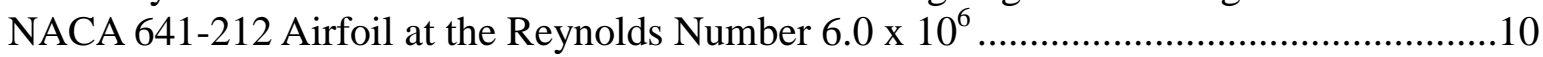

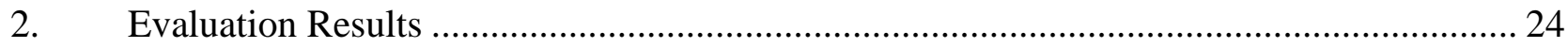

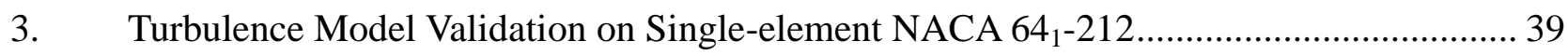

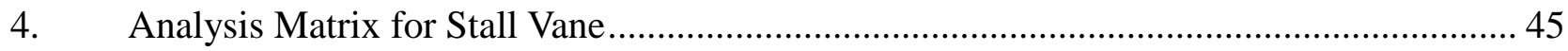

5. Analysis Matrix for Cylinder Model...................................................................... 54

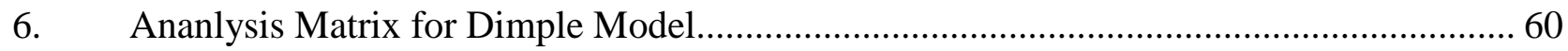




\section{LIST OF FIGURES}

Figure

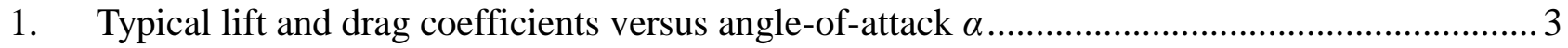

2. Effects of a leading-edge slat and a trailing-edge flap on the lift coefficient ......................... 4

3. Section lift coefficient and pitching moment coefficient against angle-of-attack of NACA

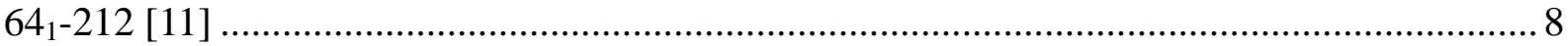

4. NACA $64_{1}-212$ with the leading-edge slat geometry notation [11] .................................. 9

5. The enlarged area shows MVGs installed on the flap of a Piper Malibu Meridian (courtesy

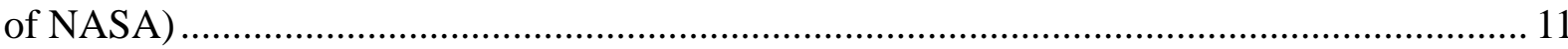

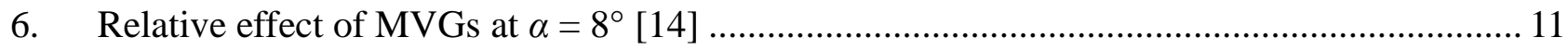

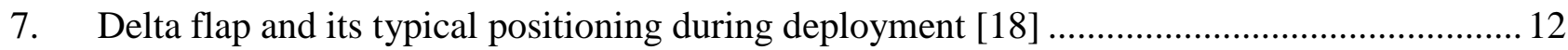

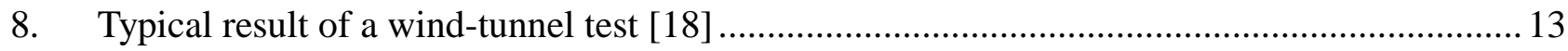

9. Arrangement of four parallel plates as a turbulent manipulator on the boundary layer [19].... 14

10. NACA 0009 skin friction reduction (right), thin plates net drag reduction (left) [21] ............ 15

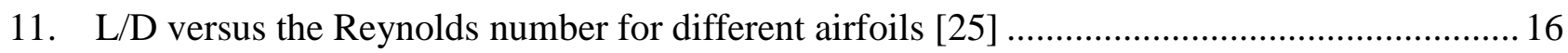

12. E398 ideal wing (left) and its inflatable wing profile (right) at $\operatorname{Re}=5 \times 10^{5} ; \alpha=0^{\circ}[24] \ldots \ldots . .17$

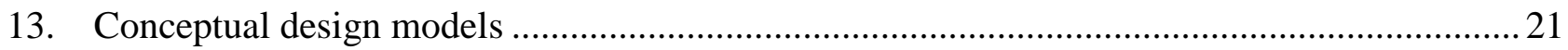

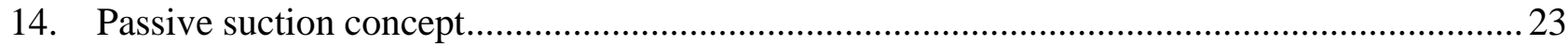

15. Boundary layer on a flat plate with laminar and turbulent boundary layers comparisons .......26

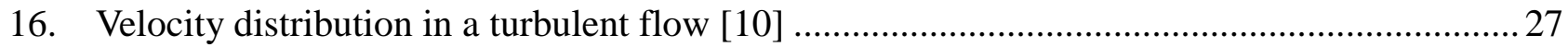

17. Boundary layer distribution at $x / c=0.95$ over the main airfoil, (A) RANS, (B) DES, (C)

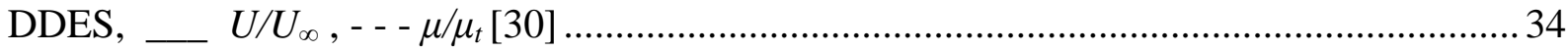

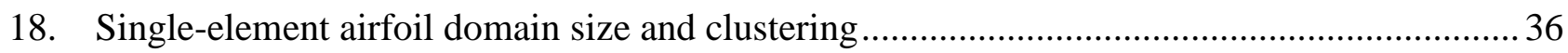

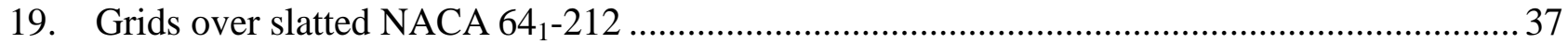

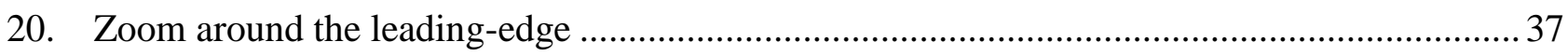




\section{LIST OF FIGURES (continued)}

Figure

21. NACA $64_{1}-212$ single-element drag polar and a quarter chord pitching moment coefficient at $\operatorname{Re}=6.0 \times 10^{6}(\mathrm{EXP}$ : experiment data [37])

22. NACA $64_{1}-212$ single-element lift coefficient at $\mathrm{Re}=6.0 \times 10^{6}(\mathrm{EXP}$ : experiment data [37])

23. NACA $64_{1}-212$ with the leading-edge slat lift and moment coefficients at $\operatorname{Re}=6.0 \times 10^{6}$ (EXP: experiment data [11]).....

24. Mesh size dependency on a pressure contour with streamlines at $\mathrm{AOA}=25^{\circ}$

25. Time step size dependency on a static pressure contour at stall

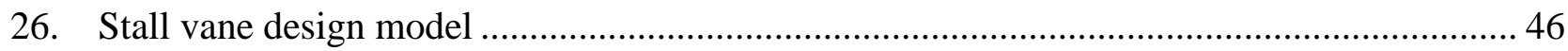

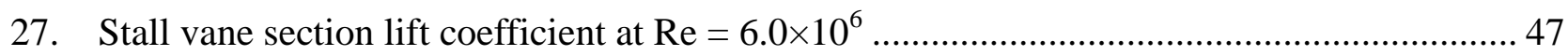

28. Stall vane lift-to-drag ratio, drag polar and quarter chord moment coefficient at $\mathrm{Re}=$ $6.0 \times 10^{6}$

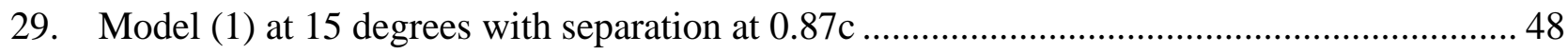

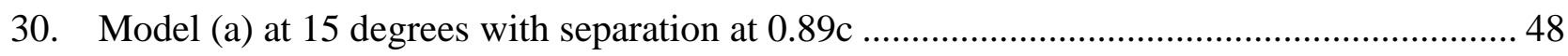

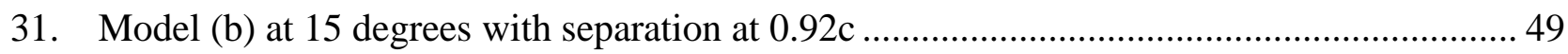

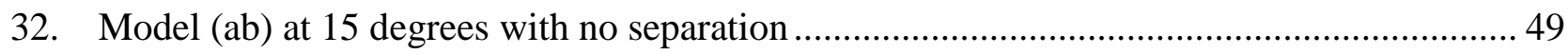

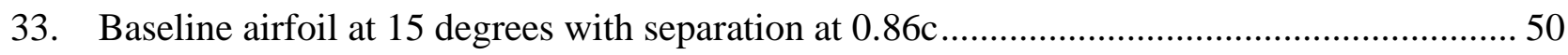

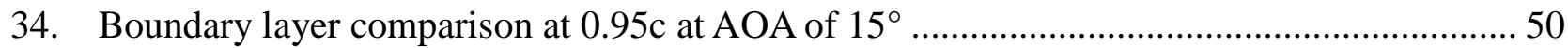

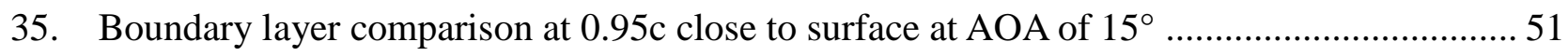

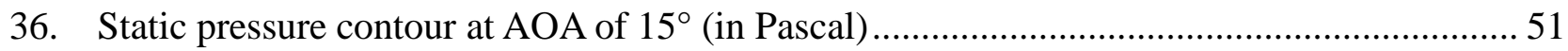

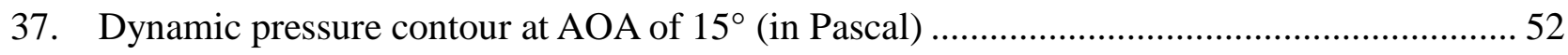

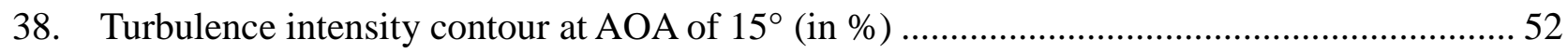

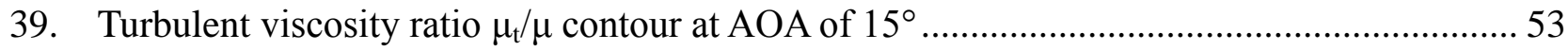




\section{LIST OF FIGURES (continued)}

Figure $\quad$ Page

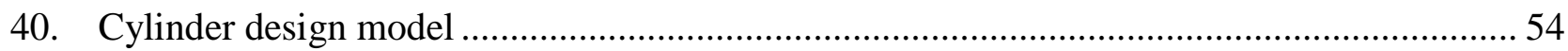

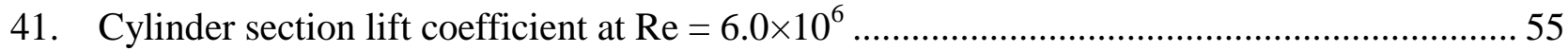

42. Cylinder lift-to-drag ratio, drag polar and quarter chord moment coefficient at $\mathrm{Re}=$ $6.0 \times 10^{6}$

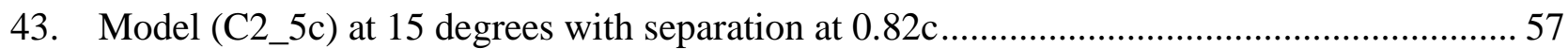

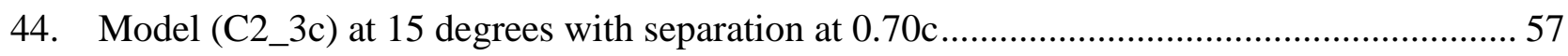

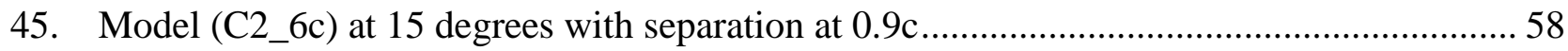

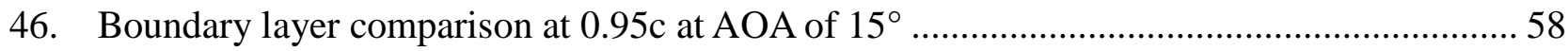

47. Model (C2_6c) turbulent viscosity ratio and turbulence intensity (\%) contours at AOA of $15^{\circ}$

48. Pressure contours over model (C2_6c) at AOA of $15^{\circ}$ (in Pascal) .................................... 59

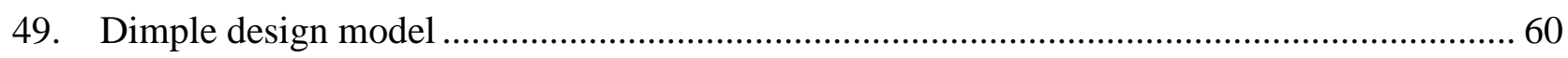

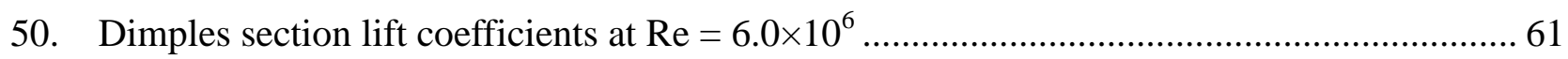

51. Dimples lift-to-drag ratio, drag polar and quarter chord moment coefficient at $\mathrm{Re}=$

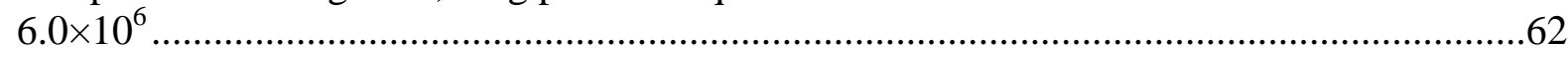

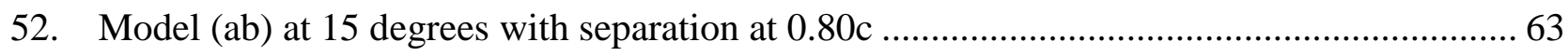

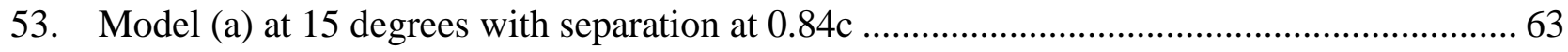

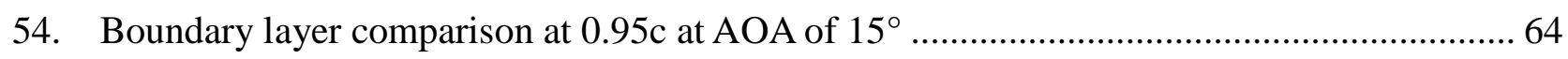

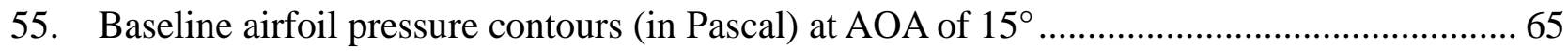

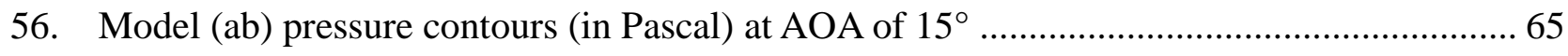

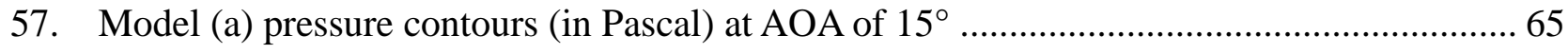

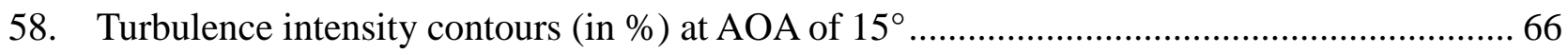

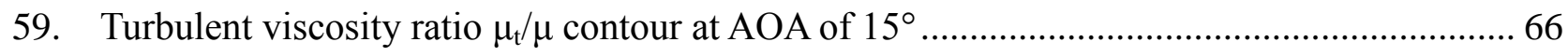




\section{LIST OF ABBREVIATIONS}

2D

Two-Dimensional

3D

Three-Dimensional

BIG BLUE Baseline Inflatable Glider Balloon-Launched Unmanned Experiment

CFD Computational Fluid Dynamics

DDES Delayed Detached Eddy Simulation

DES Detached Eddy Simulation

LEBU Large Eddy Breakup (devices)

LES Large Eddy Simulation

LTPT Low-Turbulence Pressure Tunnel

MVGs Micro Vortex Generators

NACA National Advisory Committee for Aeronautics

NASA National Aeronautics and Space Administration

RANS Reynolds-Averaged Navier-Stokes

RNG Renormalization-Group

RSM Reynolds Stress Model

SA Spalart-Allmaras

SST Shear-Stress Transport

T-S Tollmien-Schlichting waves

VGs Vortex Generators 


\section{LIST OF SYMBOLS}

\begin{tabular}{|c|c|}
\hline o & Degree \\
\hline$\alpha$ & Angle-of-attack \\
\hline$\Delta \alpha$ & Change in angle-of-attack \\
\hline$\varepsilon$ & Dissipation rate \\
\hline$\theta$ & Stall vane angle \\
\hline$\kappa$ & Turbulent kinetic energy \\
\hline$\kappa l$ & Laminar kinetic energy \\
\hline$\mu$ & Freestream viscosity \\
\hline$\mu_{t}$ & Turbulent viscosity \\
\hline$v$ & Kinematic viscosity \\
\hline$\rho$ & Density \\
\hline$\tau_{w}$ & Wall shear stress \\
\hline$\omega$ & Specific dissipation rate \\
\hline $\mathrm{c}$ & Chord length \\
\hline$d$ & Distance from the wall/dimple diameter \\
\hline$t$ & Time \\
\hline$\Delta t$ & Time step size \\
\hline$u^{+}$ & Non-dimensional wall velocity \\
\hline$u^{*}$ & Wall-friction velocity \\
\hline $\mathrm{x}$ & $\mathrm{X}$-direction distance \\
\hline$y$ & $\mathrm{y}$-direction distance \\
\hline$y^{+}$ & Non-dimensional wall distance \\
\hline
\end{tabular}




\section{LIST OF SYMBOLS (continued)}

$\begin{array}{ll}C_{D} & \text { Wing drag coefficient (lower case: section drag coefficient) } \\ C_{L} & \text { Wing lift coefficient (lower case: section lift coefficient) } \\ C_{f} & \text { Skin friction } \\ C_{m c / 4} & \begin{array}{l}\text { Quarter chord pitching moment coefficient (lower case: section pitching } \\ \text { moment coefficient) }\end{array} \\ \Delta C_{L} & \text { Change in lift coefficient } \\ L & \text { Lift } \\ M_{\infty} & \text { Freestream Mach number } \\ P_{\infty} & \text { Freestream pressure } \\ R & \text { Cylinder radius } \\ \operatorname{Re}_{T} & \text { Reynolds number } \\ \operatorname{Re}_{\mathrm{c}} & \text { Chord Reynolds number } \\ T & \text { Thrust } \\ \bar{U} & \text { Velocity-average } \\ V_{\infty} & \text { Freestream velocity } \\ W & \text { Weight }\end{array}$




\section{CHAPTER 1 \\ INTRODUCTION}

\subsection{Flow Control}

The purpose of aircraft flow manipulation, as Gad-el-Hak [1] explains, are: increasing lift, reducing drag and enhancing the mixing of mass, momentum and energy. In order to meet these objectives 1) the laminar-to-turbulent transition has to be postponed or provoked, 2) the flow separation has to be avoided or initiated, 3) the flow turbulence has to be prevented or encouraged. All these seemingly contradictory goals are interrelated. It is not difficult to look at only one performance target, the challenge is to consider the side effects and how to minimize them. Furthermore, it is essential that the resulting performance enhancing devices are simple, inexpensive, and easy to operate.

\subsection{Stall}

Flow separation is among the most significant problems in aerodynamics, and we will look closer at it first. Flow transition and turbulence will be examined together in a later section. A stall is a sudden loss of lift caused by significant amounts of flow separation. Unfortunately, it is one of the reasons for many aircraft accidents still today. A stall is among the hardest phenomenon to overcome in aerodynamics. It usually occurs immediately after the airfoil maximum lift point. However, by delaying a stall, maximum lift increases and the form drag is reduced. For instance, a $5 \%$ maximum lift coefficient improvement in landing can increase the payload size by $25 \%$ [2].

Flow separation occurs under several conditions as the fluid particles are slowed down by wall friction. For example, the adverse pressure gradient reduces the momentum of particles once retarded by the wall shear and the pressure gradient. Through the conservation of energy, some of the kinetic energy gained by the flow potential energy can be dissipated by the viscous effect inside 
the boundary layer within a favorable pressure gradient region. In the adverse pressure gradient regime, the remaining kinetic energy intends to return to potential energy but hardly overcomes the pressure rise. Therefore, fluid particles lose their momentum in the vicinity of the wall where it comes to arrest. At this point the viscous sublayer, subsequently the streamline, falls apart from the surface of the body. Thus, the boundary layer is separated. Due to a large energy loss at the separation, no boundary layer approximation is valid [1].

The separation location has a great effect on the performance of the device. For instance, a separation delay can increase the circulation around an airfoil at a high angle-of-attack and increase the lift. It also contributes to reducing the pressure drag of a bluff body and improving the pressure recovery. Experimental studies show that the flow separation control over a lifting surface greatly influences the aerodynamic properties and consequently the device's optimum performance.

In the airplane design process, it is important to evaluate performance, including a stall, by considering the airfoil lift and drag coefficients. These are fundamental quantities rather than conveniently defined expressions [3]. They contribute to the flight range, speed, payload size, power required and more. Yet, they have limits. Indeed, the largest challenge for a designer or aerodynamicist is to extend these boundaries.

Assuming an airplane is flying steady and level, the lift $L$ acts in the opposite direction to its weight $W$, and perpendicular to the incoming freestream velocity $V_{\infty}$. Thrust $T$ and $\operatorname{drag} D$ are opposed to each other and parallel to $V_{\infty}$. One can show,

$$
T=\frac{W}{L / D}=\frac{W}{\left(C_{L} / C_{D}\right)}
$$

where $C_{L}$ and $C_{D}$ are the lift and drag coefficients respectively. Equation (1.1) L/D, or the lift-todrag ratio, measures the aerodynamic efficiency of an airfoil. A higher L/D gives the airplane a 
longer range of flight. Typically, the magnitude of $L$ and $W$ is larger than $T$ and $D$. For a conventional cruise flight the lift-to-drag ratio is about 15 to 20 . If the $\mathrm{L} / \mathrm{D}$ is 15 , for example, it means by using $1 \mathrm{~N}$ of thrust to defeat $1 \mathrm{~N}$ of drag, the wing is lifting $15 \mathrm{~N}$ of weight. At fixed freestream Mach numbers (the ratio of the flow velocity to the speed of sound) and the Reynolds numbers (the ratio of the inertia forces to the viscous forces), the lift coefficient $C_{L}$ and the drag coefficient $C_{D}$ are only functions of the angle-of-attack $\alpha$. In Figure 1, note that $C_{L}$ increases almost linearly to $C_{L \max }$, after that the lift severely decreases. This result is called a stall as we explained earlier. For $C_{D}$, wings are designed so that the minimum drag is located at the cruise angle and the design lift coefficient.

There are two common properties that need to be considered in order to evaluate the effectiveness of an airfoil: the lift-to-drag ratio and the maximum lift coefficient. These parameters, among many things, determine the stalling speed, potential payload and airplane maneuverability.

$C_{L \text { max }}$ is determined by the airplane wing shape (e.g., airfoil, planform, sweep). To enhance the aerodynamic performance, most recent designs utilize devices that further increase maximum

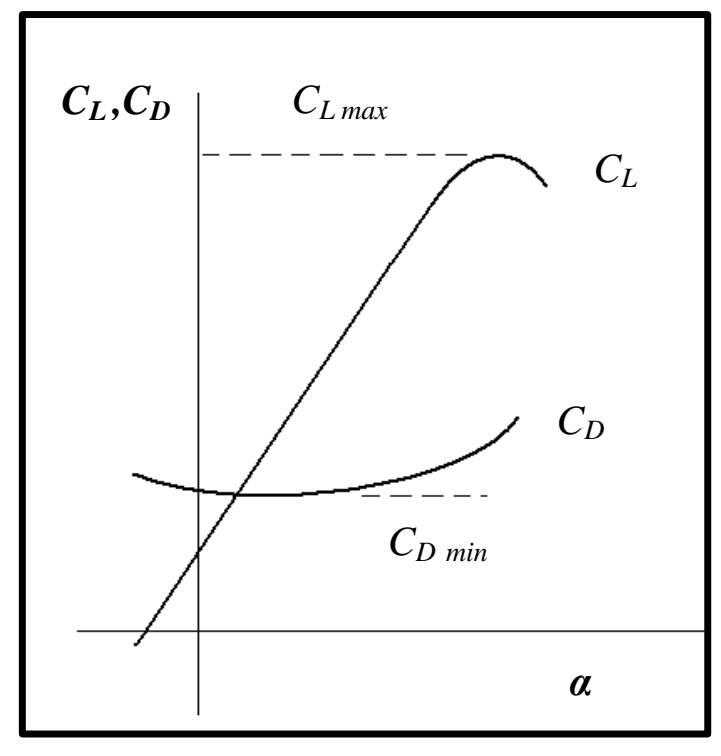

Figure 1. Typical lift and drag coefficients versus angle-of-attack $\alpha$ 
lift coefficient. Such mechanical devices like flaps and leading-edge slats are called high-lift devices. These devices are mostly used at take-off and landing; when the aircraft is at its minimum speed, and where delaying stall-speed is absolutely critical. Obviously, lift is much easier to generate as speed increases because of the higher dynamic pressures.

The trailing-edge flap is located at the aft portion of the airfoil and can be deflected at a certain angle to alter the camber. Figure 2 shows how a flap affects $C_{L}$. The amount of increase in lift coefficient is shown as $\Delta C_{L}$. The Leading-edge slat is another type of high-lift device that is located at the airfoil front. As shown in Figure 2, it allows an increase in maximum lift coefficient as the angle-of-attack increases.

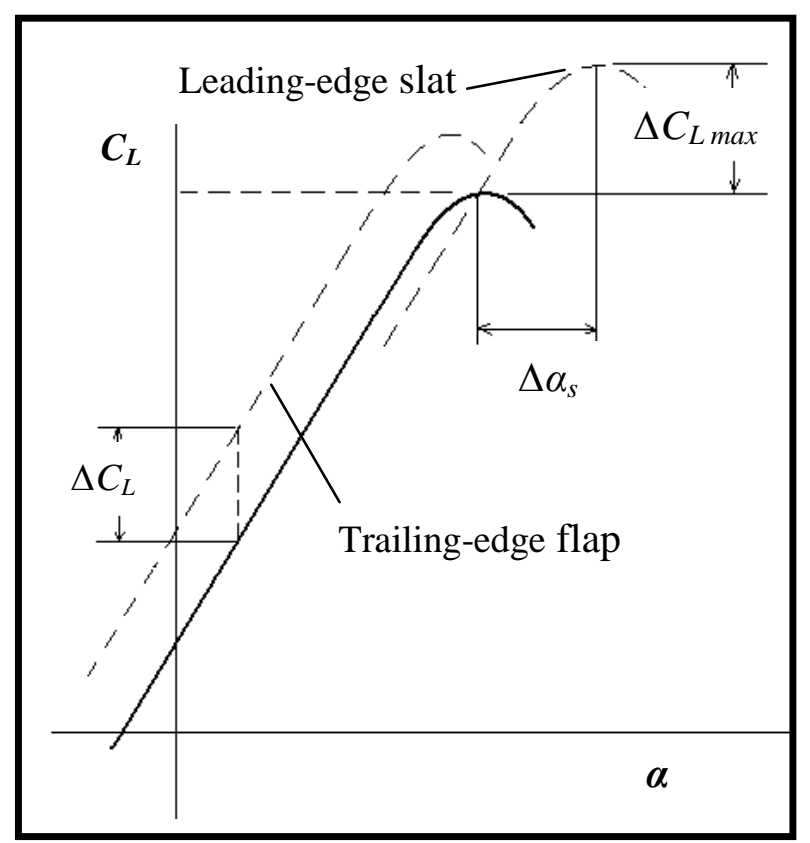

Figure 2. Effects of a leading-edge slat and a trailing-edge flap on the lift coefficient

Most leading-edge slats have a thin, curved airfoil shape in front of the main airfoil. When a slat is deployed, air flows through the gap between the slat and the airfoil's leading-edge, increasing the momentum on the upper surface [4]. The bound vortex generated by a slat also counters the circulation of the main airfoil and reduces the total pressure peak. As a result, a more uniform 
pressure distribution contributes to the stall delay. The optimum slat location was studied by Liebeck and Smyth [5]. In addition to its lift increase, slats delay the stall angle, unlike flaps that change the angle of zero lift (see Figure 2). Drawbacks of slats and flaps are that, when deployed, both cause a significant increase in drag and a large pitching moment change.

Leading-edge slats are currently used for most commercial aircraft and supersonic fighters. Slats and trailing-edge flaps are described as passive techniques, affecting momentum in the nearwall flow. No auxiliary power or control system is used to add energy continuously to the boundary layer.

Vortex generators, which will be discussed later, also affect near-wall flow by mixing freestream, 'fresh,' and high momentum flow with decelerated flow near the wall. Interestingly, other passive techniques, such as turbulence amplification with rough and wavy surfaces, can trip the boundary layer into a turbulent condition. Applications of transverse cylinders [6] and flow control rails, right above or outside the boundary layer, work similarly. Investigation of rectangular grooves and longitudinal $\mathrm{V}$ - and U-grooves has shown $[7,8]$ their effectiveness in reducing form drag at different yaw angles or even on bluff bodies. These grooves have the same influence, deflecting the flow of an outer region into the near-wall boundary layer and consequently delaying separation. Investigations made by Wallis $[9,10]$ include the leading-edge separation control by drooped nose as an alternate to using leading-edge slats.

In contrast, active methods use powered systems or devices and possibly control systems. Active suction, wall jet blowing, oscillators with embedded flaps and spoilers, acoustic excitations and many other dynamic systems have been examined and used. What is not clear is how practical their application to engineering designs is within their optimal operating range [1]. Without a doubt, the reason passive techniques are popular in applications is their simplicity. 
As was previously mentioned, the ultimate goal of flow control is to improve aircraft lift and the lift-to-drag ratio while maintaining its simplistic nature and minimizing drawbacks. It is beneficial to delay separation, since a later stall increases lift and decreases form drag. Obviously, this yields a larger lift-to-drag ratio as the angle-of-attack increases. It has been proven that early transition to turbulence helps avoid, or delay, the separation, but it also has some disadvantages.

\subsection{Turbulence Transition}

This section will take a look at the impact of flow transition to turbulence on aerodynamic performance. In the previous section we explained how delaying separation can be beneficial.

Depending on the Reynolds number, laminar flow has about $10 \%$ of the skin friction of turbulent flow. This means greater aircraft range, lower fuel costs and higher flight speed. On the other hand, turbulent flow has a higher rate mixing of mass, momentum and energy. This flow behavior helps to delay flow separation. Hence, in some cases, laminar flow is preferred, and in others, turbulent flow is desired.

For a smooth, semi-infinite flat plate in a uniform, incompressible, isotropic flow, the laminar boundary layer has a Blasius velocity profile. The laminar shear layer is sensitive to small perturbations spread in a common flow. As the Reynolds number increases and exceeds its critical value, a typical flow becomes unstable. The flow instability causes two-dimensional waves (Tollmien-Schlichting [T-S] waves) to reach non-linear amplitudes, causing three-dimensional fluctuations to dominate the flow. This situation marks the start of a transition to turbulence.

Random disturbances, such as freestream turbulence, surface roughness, vibrations, radiated sound or the combinations of these forces, cause non-linear responses. The noted phenomenon leads to a laminar shear layer transition and the development of a turbulent boundary layer. To delay transition, the following steps can be taken: First, the T-S waves need to be prevented or canceled. 
Stability modifiers reduce the growth of linear disturbances and change the shape of a velocity profile, yet they do not alter the flow stability characteristics. Secondly, providing a minimum disturbance environment is also important. The process may be delayed by using smooth surfaces, low freestream turbulence intensity and eliminating body vibration.

In comparison to laminar flow, a turbulent boundary layer is more resistant to flow separation because of its ability to mix or transport mass, momentum and energy. In some cases, mixing is far more profitable than the penalty of high skin friction. For example, an increased rate of momentum transfer can prevent boundary layer separation and increases the lift at higher anglesof-attack. Several different methods used to enhance flow mixing are:

- Increase flow instabilities

- Provoke laminar-to-turbulence transition

- Generate flow circulations or unsteady flow in laminar and turbulent regions

Some of these strategies were discussed in sections 1.4.2 and 1.4.3 (e.g., vortex generators).

\subsection{Literature Review}

In this section, some of the applications discussed previously are reviewed in greater detail. The discussion is focused on passive methods because they are easier utilized in real designs. Furthermore, the review is undertaken with the intent of laying a foundation for understanding and selecting concepts, or designs, used to improve airfoil performance.

\subsubsection{Leading-edge slat}

A series of NACA (National Advisory Committee for Aeronautics) airfoils were designed to maintain a certain velocity distribution with an emphasis on appropriate boundary layer characteristics. Interestingly, a laminar boundary layer is preserved as far as the favorable pressure gradient is extended. Thus, the location of peak suction or maximum velocity is pushed far aft, 
which contributes to the frictional drag reduction as well. The NACA 6-series airfoil attributes its low drag with a long range of laminar flow to its extensive favorable pressure gradient and the far aft transition.

The NACA Langley Two-dimensional Low-turbulence Pressure Tunnel (LTPT) has conducted numerous investigations on different airfoils. The NACA $64_{1}-212$ and NACA 65A109 airfoils, equipped with leading-edge slats at an optimum location were tested by Gottlieb [11]. Figure 3 shows the comparison between the NACA $64_{1}-212$ plain and slatted airfoils' section lift and pitching moment characteristics at the Reynolds number of $6.0 \times 10^{6}$. The leading-edge slat effect yields an increase of 0.6 in maximum lift coefficient, delaying stall by $14^{\circ}$.

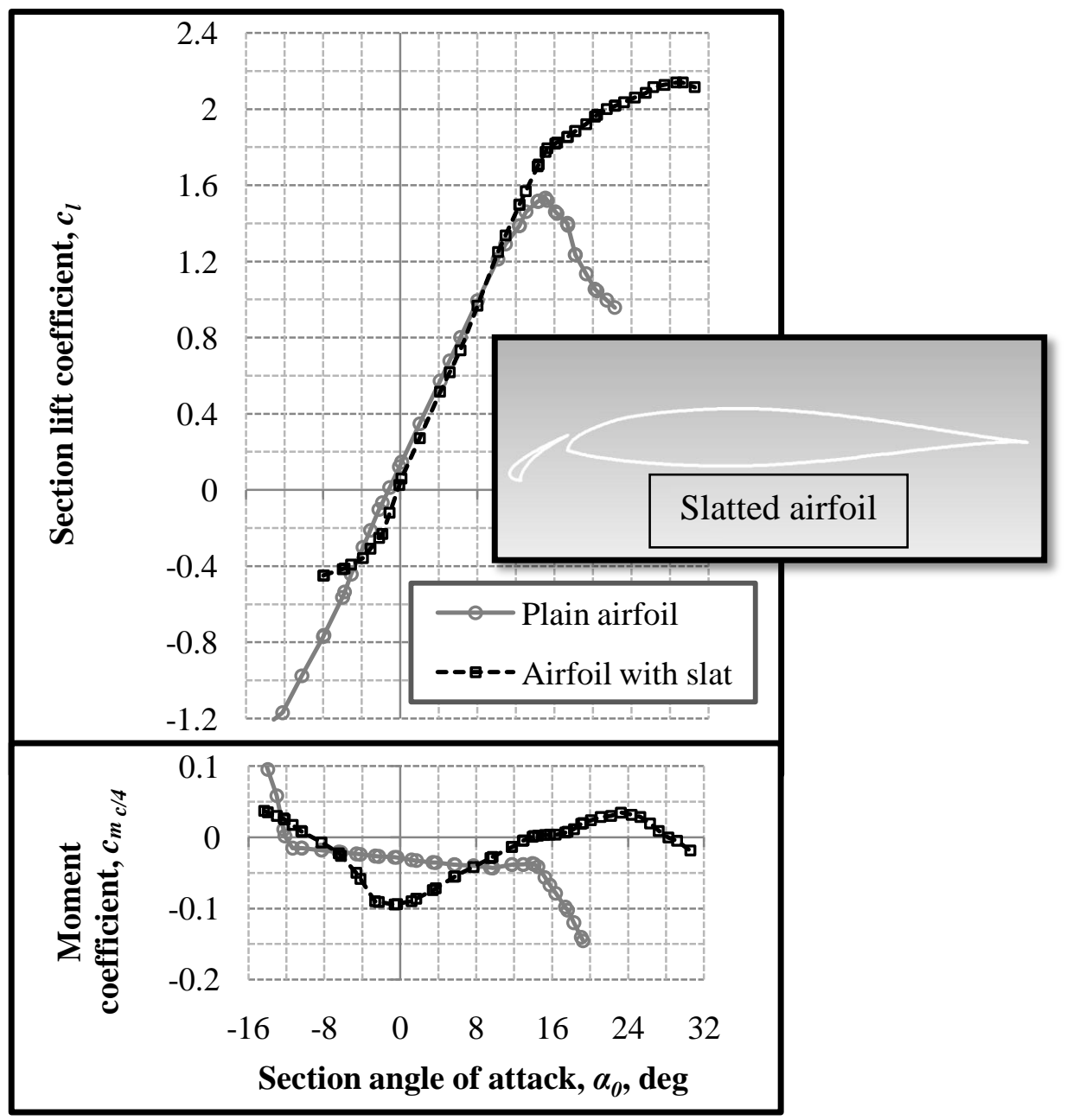

Figure 3. Section lift coefficient and pitching moment coefficient against angle-of-attack of NACA $64_{1}-212$ [11] 
The results for two types of airfoils, equipped with a $14 \%$ chord $(0.14 \mathrm{c})$ leading-edge slat and a $20 \%$ chord $(0.20 \mathrm{c})$ trailing-edge flap at the Reynolds number range of $2.0 \times 10^{6}$ to $9.0 \times 10^{6}$, are compared. This study focuses on the leading-edge slat, which has direct impact on delaying separation on the upper surface of the airfoil.

With the leading-edge slat extended (see Figure 4), stall becomes more gradual and smooth. This trend agrees with an increase in the Reynolds number for slatted airfoils. The leading-edge slat

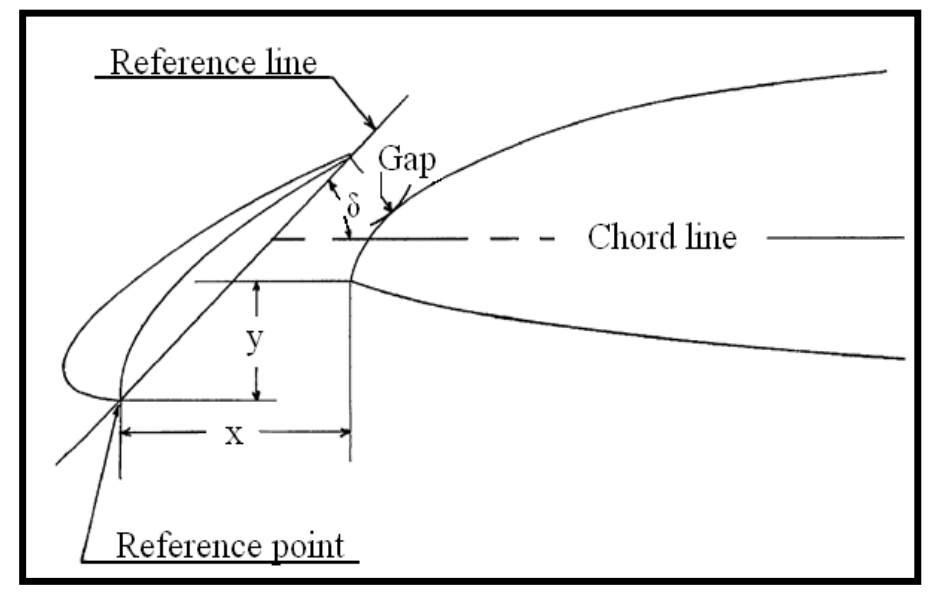

Figure 4. NACA $64_{1}-212$ with the leading-edge slat geometry notation [11]

clearly has an effect on delaying the stall angle and increasing the maximum lift coefficient. Thus, its contribution to improve the performance on landing and take-off of an aircraft is inevitable [11]. No drag measurements were provided in Gottlieb's study, but the effect of the leading-edge slat on the pitching moment coefficient at the quarter chord was examined; the aerodynamic center shifted toward the quarter chord as the leading-edge slat was deployed.

Table 1 compares the plain and slatted NACA $64_{1}-212$ airfoils with the summary of the maximum lift characteristics at its optimum slat position from Figure 4. 
TABLE 1

SUMMARY OF MAXIMUM LIFT CHARACTERISTICS AND LEADING-EDGE SLAT CONFIGURATION FOR NACA 641-212 AIRFOIL AT THE REYNOLDS NUMBER 6.0 X 106

\begin{tabular}{lcccccc}
\hline \hline & $C_{\operatorname{lmax}}$ & $\alpha_{\operatorname{Clmax}}$ & $\mathrm{x}($ chord \%) & $\mathrm{y}($ chord \%) & $\delta(\mathrm{deg})$ & Gap (chord \%) \\
\hline Plain airfoil & $\mathbf{1 . 5 5}$ & $\mathbf{1 5}$ & - & - & - & - \\
\hline Slatted airfoil & $\mathbf{2 . 1 5}$ & $\mathbf{2 9}$ & 9.9 & -6.3 & 43.3 & 1.7 \\
\hline \hline
\end{tabular}

Notes: Data is used from table 3 of reference [11]

This model serves as validation to verify the CFD solutions. References $[4,12,13]$ show similar work on fixed slats with different airfoil profiles. Wenzinger and Rogallo [13] have obtained data on several configurations, including fixed slotted leading-edge devices. In their work, forces and moments on the high-lift devices, with respect to angles-of-attack, corresponding normal forces, resultant forces, and pressure distributions are all presented.

\subsubsection{Micro Vortex Generators}

Another passive technique to control flow separation is to use vortex generators. Usually, they have a flat plate shape, set at a certain angle, vertically, against the incoming airflow on the upper surface of an airfoil. The vortex generator has been the subject of many studies and has since been applied to numerous general aviation airplanes [14-17]. Vortex generators (VGs) are fundamentally very simple, inexpensive and effective.

Figure 5 shows the small trapezoid-shaped VGs installed near the flap's leading-edge. These VGs are used to control turbulent flow at a low profile, sub-boundary layer scale, called Micro Vortex Generators or MVGs. Wind tunnel tests show that MVGs increase lift by $10 \%$, decrease drag by $50 \%$ and increase lift-to-drag ratio by $100 \%$ [14]. Another remarkable benefit of MVGs is in their capability to be stored, as the flaps are retracted during cruise flight, resulting in the 


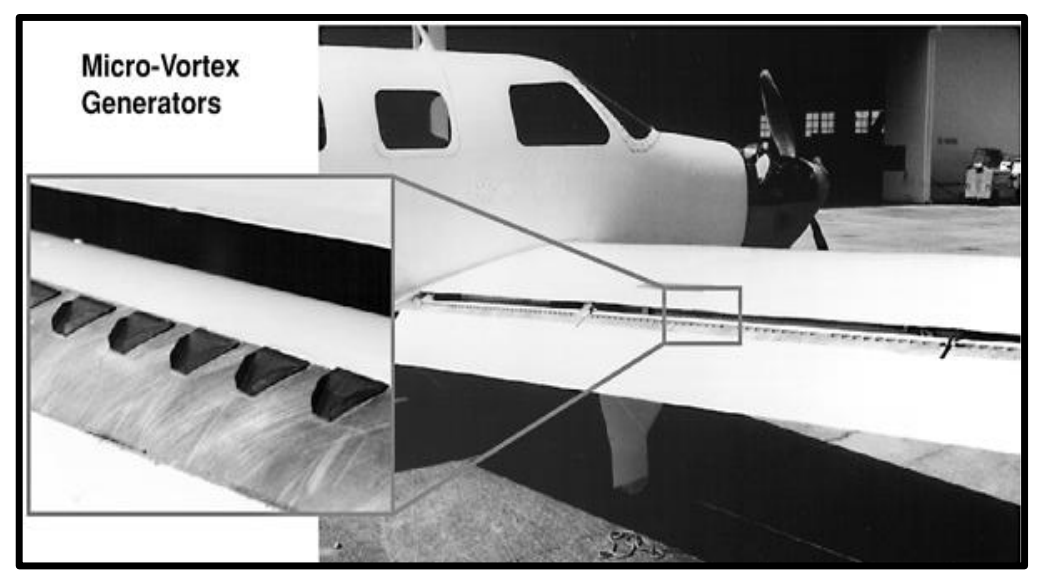

Figure 5. The enlarged area shows MVGs installed on the flap of a Piper Malibu Meridian (courtesy of NASA)

elimination of their parasite drag. Figure 6 gives the best location of the MVGs, where at $26 \%$ of the flap chord length MVGs can still be contained as the flap retracts.

For a three-element airfoil with a leading-edge slat and slotted flap, test results showed that MVGs, at 33\% of the flap chord length, created an optimal separation control effect, followed by a $25 \%$ flap chord. The test condition is at the two chord Reynolds numbers $\operatorname{Re}_{c}=5.0 \times 10^{6}$ and $9.0 \times 10^{6}$ and the Mach number $M_{\infty}=0.2$ over the range from 0 to $16^{\circ}$ angles-of-attack. Test results show a reduction of the wake size which suggests a decrease in drag and, as a result, an increase in maximum velocity. It can be concluded that MVGs enhance the total lift.

Micro vortex generators, as small as $0.18 \%$ of the chord length of the reference airfoil, can

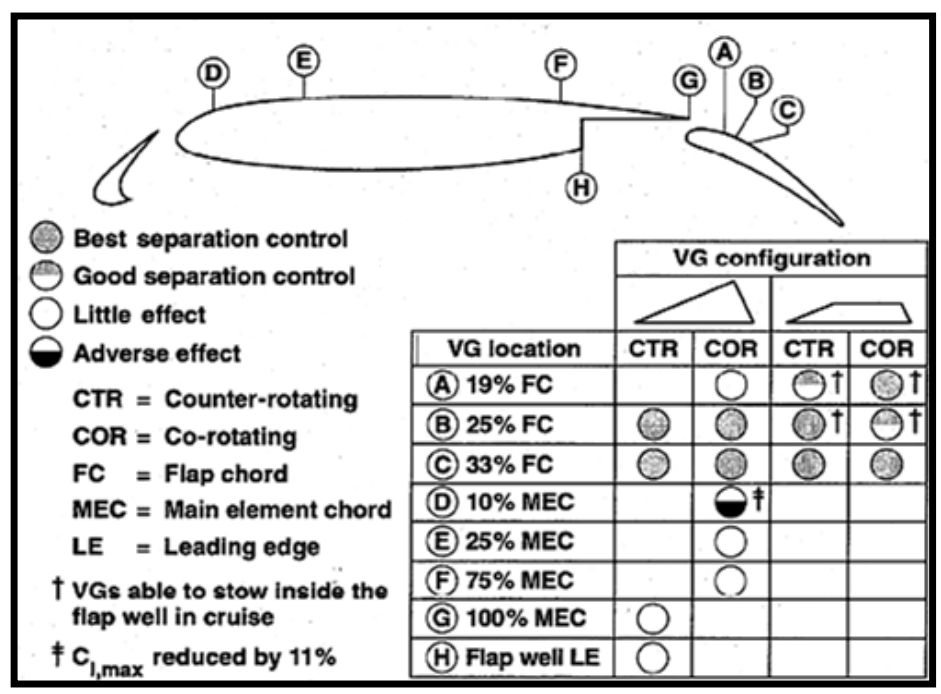

Figure 6. Relative effect of MVGs at $\alpha=8^{\circ}[14]$ 
be effective in controlling the separation of a multi-element airfoil. These devices generate chordwise, counter-rotating or co-rotating vortices on the surface of the flap, adding extra momentum to the near-wall flow. The device is effective in reducing the wake size or narrowing the separation region, but since they are installed on flap, no stall delay is detected. Accordingly, MVGs do not change the maximum lift coefficient, they reduce the drag.

\subsubsection{Delta Flap Vortex Generators}

A relatively new investigation, introduced by Nowak and Solies [18], is made on a so called delta flap. In this case, the vortex generator concept is used to generate more lift and delay stall. The wind-tunnel study includes several configurations and Reynolds numbers, at a range of angles-ofattack. The size of the delta flap is as large as $30 \%$ of the base airfoil chord $(0.30 \mathrm{c})$, with a triangular shape set close to the leading-edge.

Figure 7 shows a typical delta flap positioning with the thin, flat lens shape and sharp leading-edge. Delta flaps are deployed, as is illustrated in the left of Figure 7, with rotating arms at four different levels. They are located outside the boundary layer in such a way that they mix a high energy airflow which is not affected by the viscosity with the near surface flow.

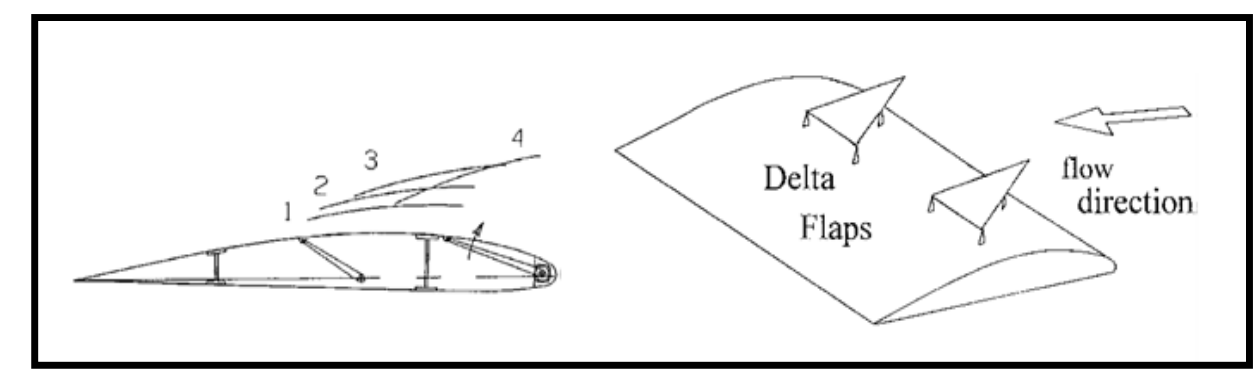

Figure 7. Delta flap and its typical positioning during deployment [18]

The general aerodynamic characteristics, in Figure 8, compare delta flaps (at level 2) with a baseline wing (at level 0). A Delta flap, at its optimum deployment, increases the maximum lift coefficient and maintains it for a long range of angles-of-attack. No sudden lift reduction at stall is 


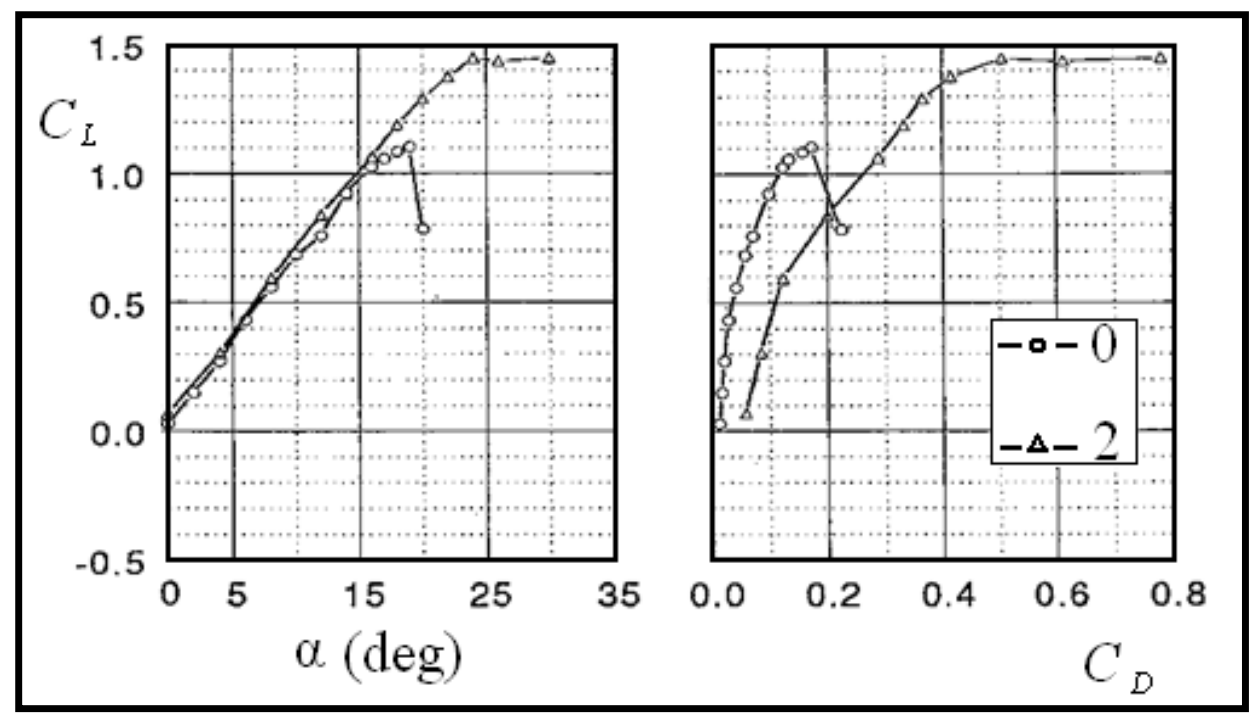

Figure 8. Typical result of a wind-tunnel test [18]

observed in addition to the 5 7 degrees stall delay. It is evident that at high angles-of-attack drag also increases significantly. The right-hand plot, in Figure 8, shows the drag polar of two different configurations. No pitching moment data is available in this report, although the delta flaps are responsible for a "moderate pitch-up moment", changing the negative moment of the baseline into a positive. Meanwhile, the effect of the Reynolds number has little significance in changing aerodynamic coefficients. Nevertheless, only a flow of Reynolds number up to $5.9 \times 10^{5}$, which is a relatively low number, is able to stay attached throughout the range of angles-of-attack. This indicates that for the higher Reynolds number, close to typical cruise conditions, this type of device is not suited.

\subsubsection{Turbulence Manipulator and Large Eddy Breakup Device}

A turbulent boundary layer can tolerate, and is more resistant to adverse pressure gradients, when compared to a laminar boundary layer. Thus, laminar-to-turbulent transition advancement may be beneficial in some cases. This goal may require the use of a boundary layer tripping device such as: surface roughness or waviness, mass transfer and mixing, and body vibrations. 
Although a turbulent boundary layer has significantly higher skin friction, devices such as turbulence manipulators can reduce their drag penalty. This section will discuss Large Eddy Breakup Devices (also known as LEBU devices) [1], considering their potential as an individual or in mutual interrelation with other separation control devices such as VGs.

Croke et al provide extensive examinations on the effects of turbulent boundary layer manipulators [19]. The purpose of the manipulator is to reduce the surface drag or skin friction caused by large eddies in the turbulent boundary layer. The parallel plates, in Figure 9, are effective in lowering the turbulence intensity. The removal of the large-scale structures, in the turbulent

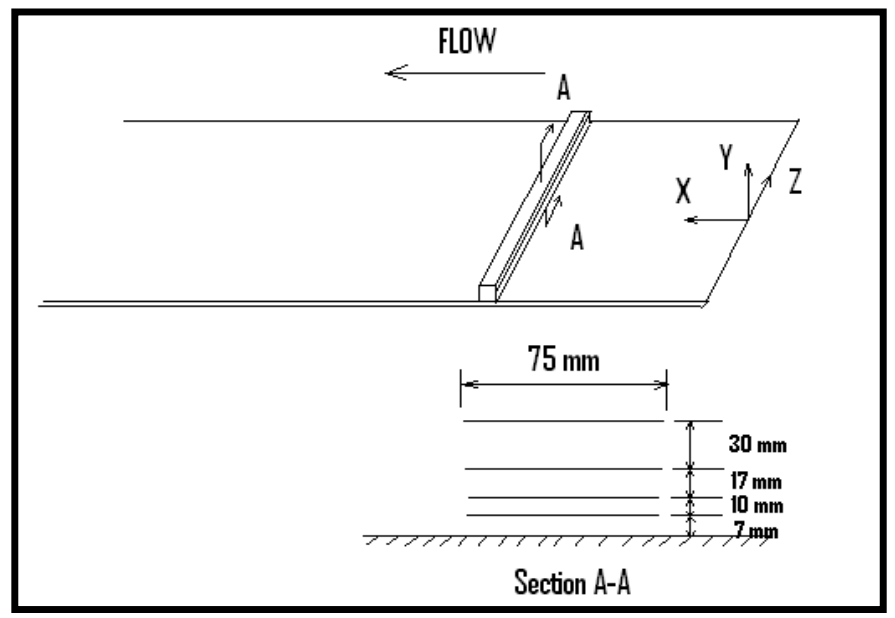

Figure 9. Arrangement of four parallel plates as a turbulent manipulator on the boundary layer [19]

boundary layer, by LEBU devices helps reduce the drag for a distance up to 70 boundary layer thicknesses downstream. The manipulator multi-spacing option eliminates the different sizes of large-scale structures, or eddies, in the boundary layer. The streamwise distance from the manipulator to the leading-edge was set to $x_{0}=1.4(\mathrm{~m})$, so that the fully developed turbulent boundary layer could grow naturally up to the time it gets to the manipulator. Skin friction decayed approximately by $30 \%$ immediately downstream of the manipulator, in addition to a $10 \%$ reduction 
in turbulence intensity shown in hot-wires surveys. The preliminary investigation here is based on a fixed location which leaves it open for further examinations at several distances downstream.

There are two reasons for the reduction of skin friction: 1) The boundary layer momentum deficit is increased, and 2) the large-scale eddies are modified. Studies show that turbulence fluctuating velocity is reduced by up to $20 \%$ on LEBU device application in comparison to an undisturbed flat plate. LEBU [20-23] devices are usually thin plates or airfoil shapes placed parallel to the flow direction. It is most effective to place LEBU devices in tandem, 6 to 10 chord lengths apart, and near to the border of the turbulent boundary layer.

LEBU devices are positioned where the turbulent boundary layer thickness is approximately $25(\mathrm{~mm})$. The extended test region is $3.0(\mathrm{~m})$ downstream, or about 120 boundary layer thicknesses $\left(\delta_{0}\right)$. The distance from the device is stated as $x_{0}$ and the distance of measurements, from the leading-edge, is shown as $x$. Note that subscript 'ref' implies a base line configuration in Figure 10.

The total net drag reduction, including skin friction $C_{f}$ of the test plate and the LEBU devices, of $10 \%$ is reported by Anders [21, 22]. Net drag reduction is shown in Figure 10 for the both low and high Reynolds numbers for the thin plate devices. The drag reduction for the high Reynolds number $\left(\operatorname{Re}_{\mathrm{c}} \approx 7.5 \times 10^{4}\right)$ is more than for the low Reynolds number $\left(\operatorname{Re}_{\mathrm{c}} \approx 3.1 \times 10^{4}\right)$ case

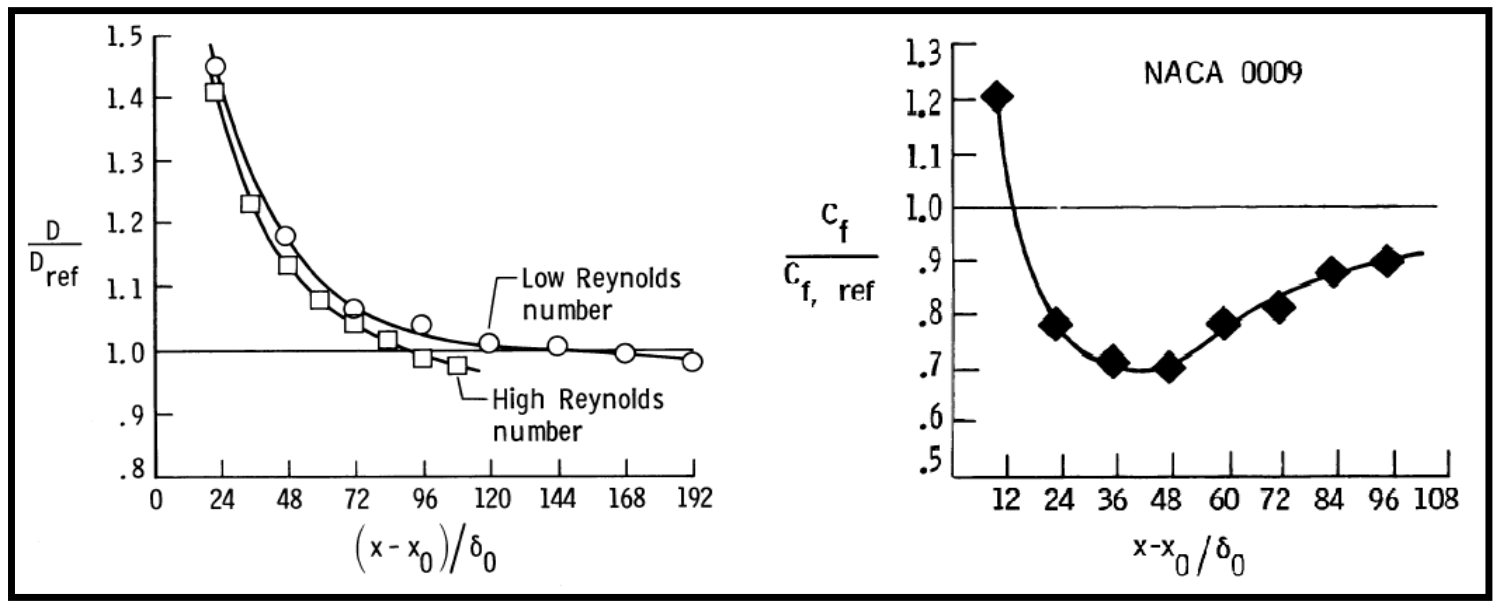

Figure 10. NACA 0009 skin friction reduction (right), thin plates net drag reduction (left) [21] 
throughout the region. Up to a non-dimensionalized distance of 96 boundary layer thicknesses, the net drag reduction is effective for the high Reynolds number. In Figure 10, downstream of a tandem set of NACA 0009 (symmetric airfoil) the local skin friction is given. A maximum of 30\% skin friction reduction is measured at 40 boundary layer thickness downstream. The results show that the airfoil shape LEBU is as effective as a thin flat plate device used to reduce the skin friction.

\subsubsection{Bumpy Inflatable Airfoils}

The BIG BLUE (Baseline Inflatable Glider Balloon-Launched Unmanned Experiment) is a University of Kentucky [24] project that's purpose is to flight-test a high altitude aircraft with inflatable wings. The flight is at high altitudes where the air density is low, and since the Reynolds number is a function of density, this flight is designed for a low Reynolds number condition. Considering other test constraints, the aircraft has to utilize inflatable wings at the given flight condition.

Figure 11 explains the lift-to-drag ratio (L/D) versus the Reynolds number for rough and smooth airfoil surfaces [25]. A typical smooth airfoil performance drops at low- $\operatorname{Re}\left(\operatorname{Re} \leq 10^{5}\right)$. In contrast, rough surface airfoils have better L/D for the same flight condition. For the rough airfoils,

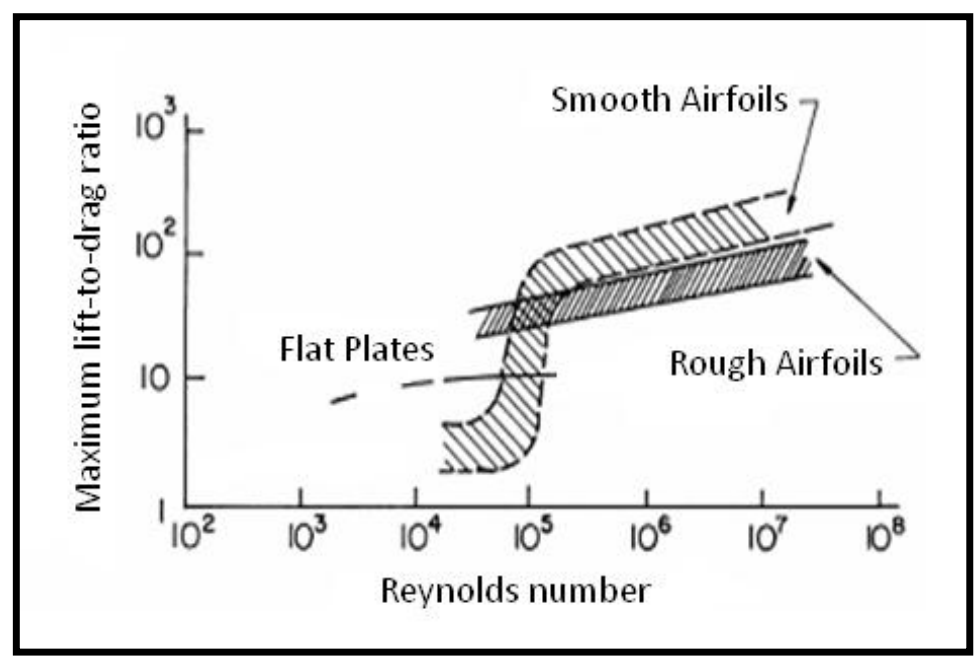

Figure 11. L/D versus the Reynolds number for different airfoils [25] 
the flow is normally tripped, and it becomes turbulent, thus it is more resistant to the flow separation. Drag also increases as the Reynolds number increases. For a smooth surface airfoil at low-Re, flow is laminar with a low profile drag, but flow separates easily as the angle-of-attack increases.

Smoke-wire flow visualization results from reference [24] are shown in Figure 12, for $\operatorname{Re}=$ $5.0 \times 10^{4}$ at an angle-of-attack of zero degrees. The flow stays attached further toward the trailingedge of the inflatable E398 airfoil (right). The separation is smaller on the 'bumpy,' inflatable airfoil than on the smooth surface in the low-Re range. The aerodynamic characteristics are even better in the range of high angles-of-attack.

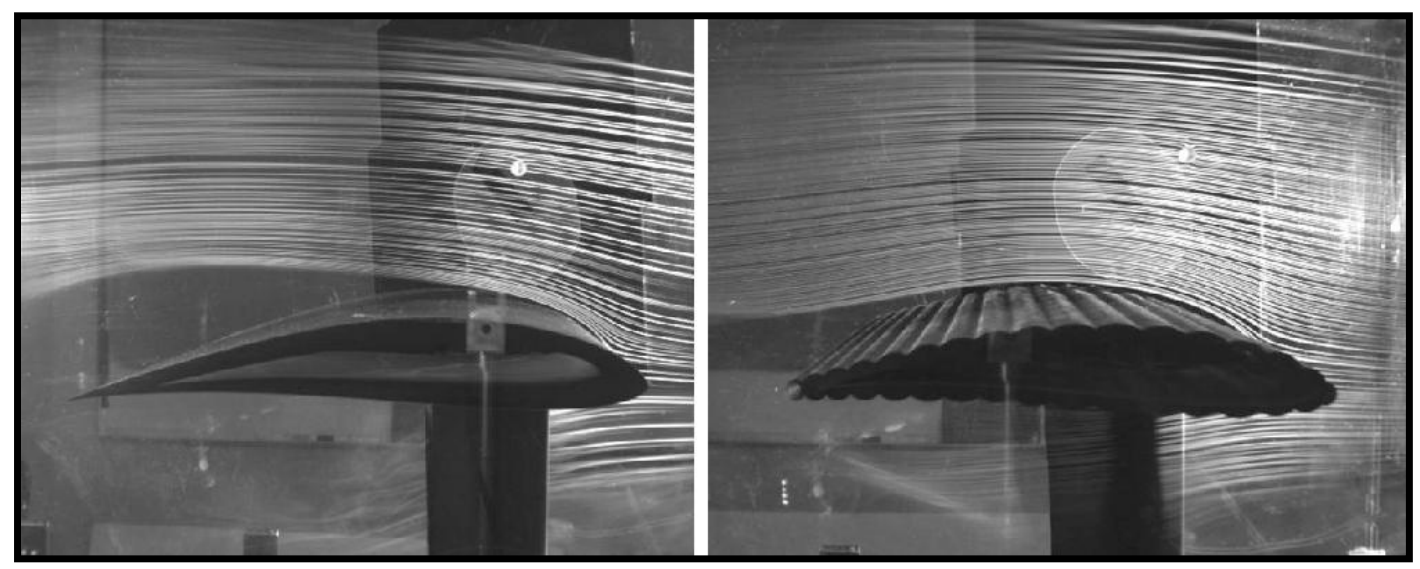

Figure 12. E398 ideal wing (left) and its inflatable wing profile (right)

$$
\text { at } \operatorname{Re}=5 \times 10^{5} ; \alpha=0^{\circ}[24]
$$

Lift and drag measurements for the Reynolds numbers range $\left(\operatorname{Re}=1.56,2.5\right.$ and $\left.3.0 \times 10^{5}\right)$ show results for $C_{l \max }$ and $\mathrm{L} / \mathrm{D}$ to be the best at the lowest measured Reynolds number. At $\mathrm{Re}=$ $1.56 \times 10^{5}, C_{l \max }$ was 1.33 and L/D was 23 , whereas at $\mathrm{Re}=3.0 \times 10^{5}, C_{l \text { max }}$ was 0.95 . No significant loss of lift (stall) is observed prior to 18 degrees of angle-of-attack at $\operatorname{Re}=1.56 \times 10^{5}$. 


\subsection{Problems and Goals}

In this work, the focus is on how to increase airfoil efficiency. For external wall bounded flow, like an aircraft or submarine exterior surface, boundary layer manipulations become critical in an effort to enhance performance. It is known that flow separation leads to loss of lift (stall). If the boundary layer is turbulent, the flow is more resistant to separation, and more lift can be generated due to a delayed stall. When flow separates, a loss of lift and an increase in form drag cannot be avoided. The goal is to postpone separation so that lift is increased and drag is reduced.

Two of the most practical passive methods to delay airfoil stall are leading-edge devices and vortex generators. Unfortunately each of these methods has problems. For instance, if the slat is fixed, then its profile drag is an issue during high-speed cruise flight. Retractable devices require extra components and power for deployment that makes the mechanism complicated. Maintenance and manufacturing processes need to be considered as well as total system weight. Also, changes in leading-edge geometry caused by: icing, bugs and dust accumulations have a large impact on the flow over the main airfoil. As a result, drag increment cannot be ignored.

Vortex generators (VGs), in contrast, are simpler, but several parameters need to be considered for the design process: cross section shape or camber, yaw angle, height against the boundary layer thickness, aspect ratio and spacing. Once set and fixed to the surface, they cannot be adjusted for different flight conditions. Conventional VGs cause a considerable amount of parasite drag. Most of the VGs are not retractable causing a reduction in cruise performance.

Considering alternative concepts that delay the flow separation, the goal of this research are:

1. Study simple passive devices that delay separation for a few degrees, with resulting higher lift-to-drag ratio

2. Minimize impact on pitching moment 
3. Consider typical flight condition applications, where the freestream Reynolds number of $6.0 \times 10^{6}$

4. Utilize Computational Fluid Dynamics (CFD) with a suitable turbulence model to study the new concepts

Experimental data for a NACA 6-series airfoil is used to validate the accuracy and consistency of the CFD module and meshing techniques. Results (e.g., lift-to-drag ratio, streamline contour and pressure contour) for each conceptual design are compared against single-element airfoil. Suggestions are made for the future optimizations and testing. 


\section{CHAPTER 2}

\section{NEW DESIGN CONCEPTS AND SCREENING}

In general, coherent and conceptual design of flow control devices are usually preferred to a heuristic process, that is, a trial-and-error approach. Based on the discussion in previous sections, flow separation control can be obtained by two different techniques; mixing augmentation (e.g., VGs) and flow redirection (e.g., leading-edge slats). Nevertheless, one must minimize the penalty factors such as drag, pitching moment and complexity of the device within the design constraints. These limitations need to be screened at a conceptual level, collectively with device advantages and weaknesses, all the while working to avoid the excessive model evaluation.

The first step is to define each design evaluation factors. In order to assess conceptual designs, we have selected eight factors for each model. These elements represent the feasibility of a device for application and will be compared with the leading-edge device as a base line. Assuming the significance of each factor is similar to each other, one can intuitively score each element with the following definitions:

1) Weight: the total weight of the device and the airfoil, whether the device is external or employed within the airfoil. The poorer the device becomes as the weight increases.

2) Surface area: the total surface area of the device adds to the basic geometry, which causes drag. The poorer the device becomes as the size of the frontal area increases.

3) Stress/Force: the total force, excluding drag, which a device produces in flight condition when deployed. This force can include pitching moment and other stresses on the device. The poorer the device becomes as the external forces increase.

4) Stability: Stability refers to a device's aeroelasticity characteristics, such as the tendency of fluttering. The poorer the device becomes as the stability decreases. 
5) Maintainability: The period of time in which the device maintains its efficacy and how often the device needs to be examined for maintenance. It also measures the impact that a device failure or malfunction can impose on airfoil performance. The superior the device becomes if has less failure impact and maintenance required.

6) Deployment: the simplicity of the device's deployment, if necessary. If a mechanical device required using external power for operation, the device becomes poor.

7) Manufacturing: the difficulty in design and manufacturing is another important process that needs to be considered. The superior the device becomes with an easier manufacturing.

8) Variable factors: determinants such as $x-y$ location, length and shape are all examples of changeable factors. An increase in the variable numbers cause more interaction against one another. Each additional variable adds to the design complexity.

When considering the effectiveness of a device, the factors above facilitate the reduction of design complexity at preliminary level. The simplicity of a device has a large effect on the total scoring. Six conceptual designs, introduced in Figure 13, were evaluated. Each model is scored in a

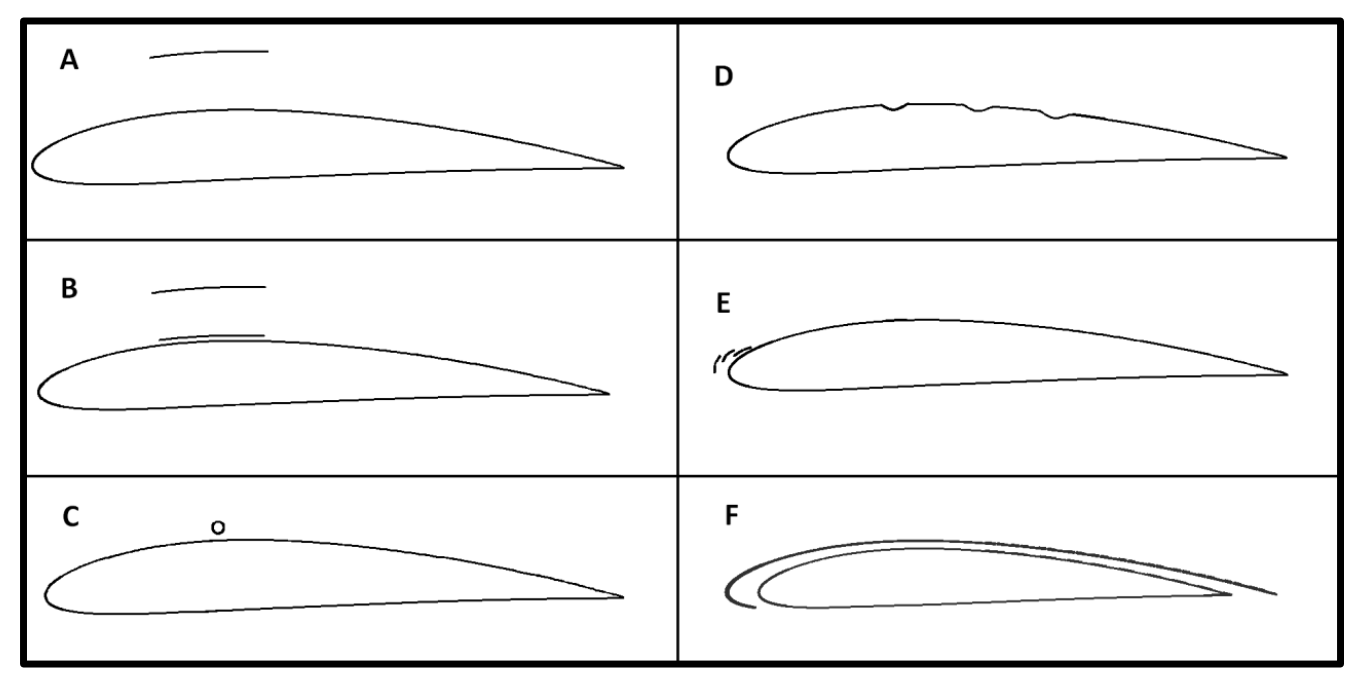

Figure 13. Conceptual design models 
scale of five, accordingly by the factors presented above. Two other aerospace engineers also scored these configurations. The three highest scoring models will be chosen for the final evaluation stage.

The six models presented in Figure 13 are either exclusive or modified configurations of existing devices. Details and description of each model is presented here.

A) Stall Vane: a Stall vane is similar to leading-edge slats, but instead of at the front, it is located at the upper surface, close to the maximum thickness of the main airfoil. It is intended to prevent separation initiated from the trailing-edge, by bending the flow toward the trailing-edge. Additionally, it aims to smooth the pressure distribution on the upper surface, by reducing the suction peak prior to the pressure recovery region. It can be either deployed or fixed at any flight condition.

B) Multi-stall Vanes: this device has a similar purpose to the Stall vane and LEBUs introduced in chapter 1. It is hypothesized that the inner plate of multi-spacing vanes eliminates large-scale eddies at the turbulent boundary layer while the outer working as the Stall vane. Drag reduction of the main airfoil has been proven in previous studies.

C) Cylinder: a cylindrical profile at the upper surface acts as a turbulator. It is hoped that this device will initiate early transition and enhance mixing. Although, an increase in drag can be expected, the drag can be reduced with a smaller diameter.

D) Dimples: such devices can be found on a golf ball (but at a lower Reynolds number) and can be considered on an airfoil. A dimple performs as a turbulator with minimum drag. The device is implemented on the upper surface of the airfoil. The number and diameter of the dimples are critical variables, as well as the chord-wise location. However, rough surfaces are more effective at the low Reynolds number, as we showed in Figure 11. 
E) Leading-Edge Vanes: this device is similar to the leading-edge slat, but it is simpler and has less effect on drag. Multiple vanes can guide the flow efficiently over the upper surface at several boundary layer thicknesses. Mass flow can be adjusted by the vane spacing and position, although variable factors increase with the number of slats. It is possible this device, at its optimum, can be fixed with minimum penalty at cruise.

F) Passive Suction: airfoil boundary layer suction is equivalent to the elimination of viscosity effect or the shear layer. This device contains two airfoils, the inner and outer surfaces (see Figure 14). Outer surface can be modified for specific cruise condition with the designated drag bucket and lift coefficient. The inner layer is designed for highlift with a larger radius at the leading-edge for take-off and landing purposes. As the angle-of-attack increases, a passive suction device activates. The reverse flow on the lower surface, close to the leading-edge, opens the valve between two layers and flow runs through the layers. The effect of convergent channel flow encourages the flow to speed up, as it reaches to the trailing-edge. Consequently, dynamic pressure rises as the

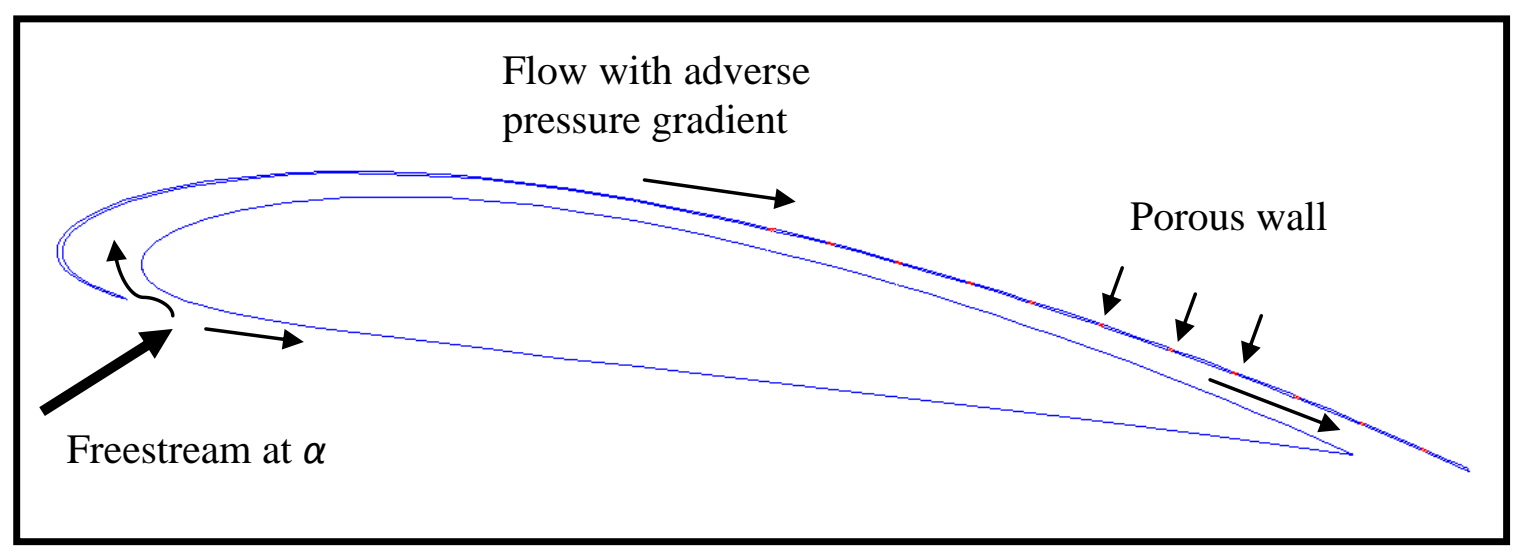

Figure 14. Passive suction concept

static pressure drops. On the other hand, flow on the upper surface faces a large adverse pressure gradient with a possible flow separation that causes static pressure to increase. 
The porous surface that divides two layers close to the trailing-edge attempts pressure equilibrium by sucking in the flow into the inner section.

Complied screening results are presented in tables 2 and 3. Amazingly, the best three configurations matched for all the screeners. Stall vane, cylinder and dimples were chosen among all six models and will be evaluated by a computational fluid dynamics tool. Note that an airfoil with a leading-edge slat is the base line, with a value of 3 , for each the categories.

TABLE 2

EVALUATION RESULTS

\begin{tabular}{|c|l|c|c|c|c|c|c|}
\hline & Factors/Configures & A & B & C & D & E & F \\
\hline $\mathbf{1}$ & Weight & 3 & 2 & 4 & 5 & 1 & 1 \\
\hline $\mathbf{2}$ & Surface Area & 3 & 2 & 4 & 4 & 2 & 2 \\
\hline $\mathbf{3}$ & Force/Stress & 3 & 2 & 1 & 4 & 2 & 2 \\
\hline $\mathbf{4}$ & Stability & 3 & 1 & 2 & 5 & 2 & 3 \\
\hline $\mathbf{5}$ & Maintainability & 3 & 3 & 3 & 5 & 2 & 2 \\
\hline $\mathbf{6}$ & Deployment & 2 & 1 & 2 & 5 & 1 & 1 \\
\hline $\mathbf{7}$ & Manufacturing & 3 & 2 & 3 & 5 & 2 & 2 \\
\hline $\mathbf{8}$ & Variable Factors & 3 & 1 & 3 & 4 & 2 & 3 \\
\hline & Screener 1: Total & $\underline{\mathbf{2 3}}$ & 14 & $\underline{\mathbf{2 2}}$ & $\underline{\mathbf{3 7}}$ & 14 & 16 \\
\hline \hline
\end{tabular}




\section{CHAPTER 3}

\section{NUMERICAL SCHEME}

The fundamental laws of fluid dynamics apply to all fluid motion. Conservation of mass, momentum and energy are partial, non-linear differential equations requiring proper boundary and initial conditions. The extreme complexity of the involved governing equations does not always allow one to evaluate rational designs. At the beginning of this chapter, the boundary layer is introduced to help identifying the most appropriate numerical scheme and meshing technique for this investigation.

\subsection{The Boundary Layer}

The boundary layer is where the velocity gradient normal to the surface $\partial u / \partial y$ is large. Although the viscosity is small in most cases, it has a significant role in the viscous shear stress $\tau=$ $\mu \partial u / \partial y$. The boundary layer has, mainly, two different types of flow: laminar or turbulent.

A laminar boundary layer, or layered flow, has a different velocity in each layer of fluid, but only in the flow direction. There is no exchange of fluid particles within the layers that are normal to the surface direction. Flow over a thin flat plate is an example of the simplest application of the boundary layer. Flow along the flat plate at zero incidence and boundary layer development is illustrated in Figure 15. The sketch shows the characteristics of the laminar boundary layer in comparison to the turbulent boundary layer with corresponding thicknesses.

The physical nature of turbulent flow has two principle properties at the high Reynolds number. First, is its random motion process, and second, is its wide range of continuum in space and time scales. Turbulence produces random fluctuations, in which details are hard to predict. However, its statistical properties simulate the flow averages and probability distributions. 


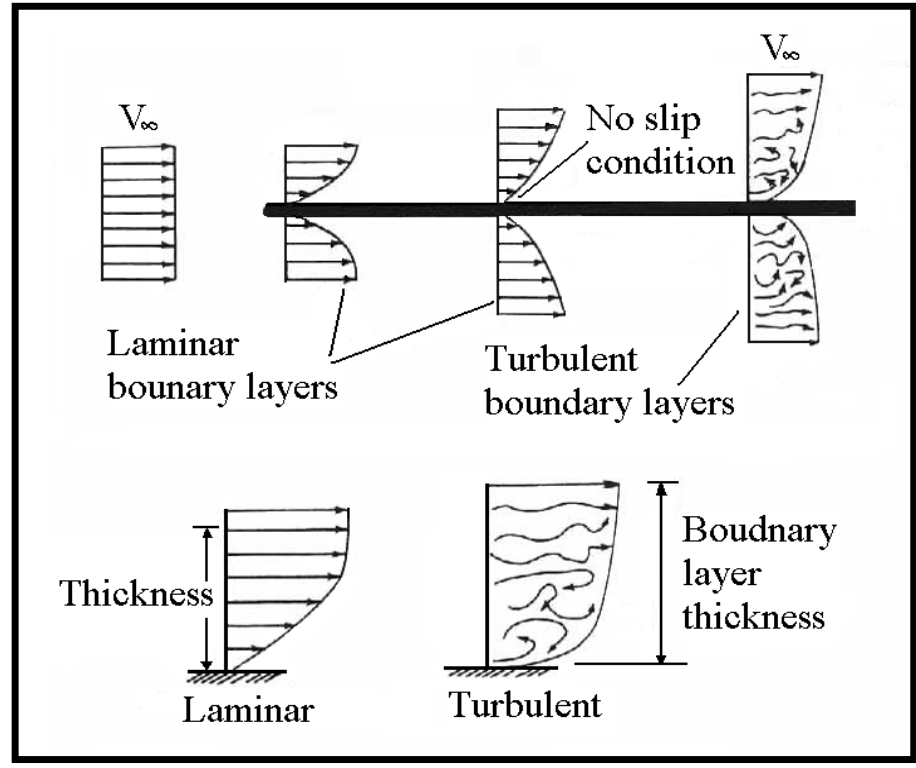

Figure 15. Boundary layer on a flat plate with laminar and turbulent boundary layers comparisons

There is no exact solution for the turbulent boundary layer, even for a simplified model of a flat plate. Near the surface, velocity is mostly influenced by the viscosity or wall shear stress, $\tau_{w}$, and the distance from the wall $y$. In this region, the velocity profile is linear depending on $y$, hence

$$
\tau_{w}=\mu \frac{\partial \bar{U}}{\partial y}=\mu \frac{\bar{U}}{y}
$$

If we define $u^{*}=\sqrt{\tau_{w} / \rho}$ as wall-friction velocity and $\bar{U}$ as velocity-average, then

$$
u^{+}=\frac{\bar{U}}{u^{*}}
$$

and the distance from the wall can be expressed in a non-dimensional form of

$$
y^{+}=\frac{y u^{*}}{v}
$$

Now, notice that $y^{+}$is in form of the Reynolds number. Using equations (3.2) and (3.3), substituting into equation (3.1), we have

$$
\tau_{w}=\mu \frac{u^{+} u^{*}}{\left(y^{+} v\right) / u^{*}}=u^{+} / y^{+} \rho u^{* 2}
$$


It is clear that in the viscous sublayer, where velocities are too small and viscous force dominates the flow, turbulence has no influence, thus, $u^{+}=y^{+}$.

A typical velocity distribution across the turbulent boundary layer is presented in Figure 16 with its linear vertical axis and the logarithmic horizontal axis. The inertial sublayer connects two distinct regions: the viscous sublayer and the outer layer.

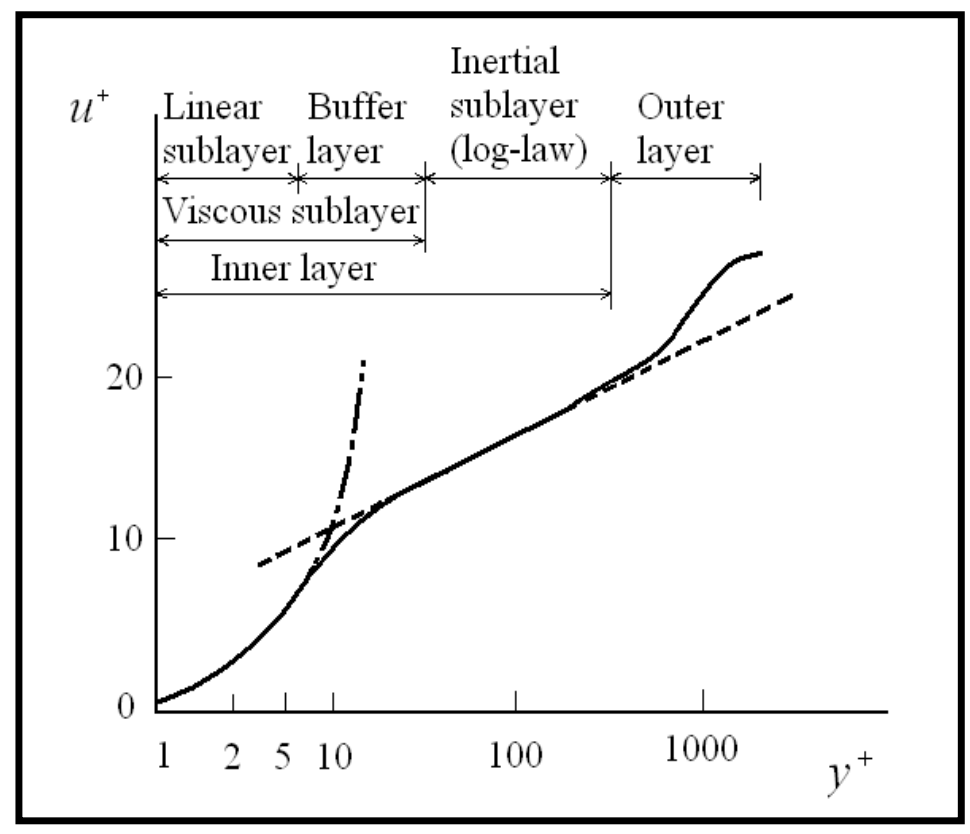

Figure 16. Velocity distribution in a turbulent flow [10]

To find accurate velocity gradients, near the wall in computational fluid dynamics, a sufficient amount of grid points are necessary in the linear sublayer. Most references suggest that $y^{+}$ should be 5 or less and in most cases $y^{+} \approx 1$ is recommended [10].

\subsection{Computational Fluid Dynamics}

There are two separate approaches to evaluate the performance of our conceptual designs. Wind tunnel testing is an experimental method used to measure the aerodynamic forces and moments on an airfoil. Experimental data are highly reliable, but it can become costly and time consuming as the run number increases on several different models. A second approach is a 
numerical solution used to solve the governing equation showed in previous chapter. The simplifications of non-linear equations solved using modern computers, makes Computational Fluid Dynamics (CFD) an effective and efficient alternative.

CFD methods are becoming even more valuable as the accuracy and simplicity of codes and interfaces develop. CFD makes it possible to solve complex flow fields that are; unsteady, compressible, viscous, turbulent and separated. Even without geometrical simplifications for an unsteady, two-dimensional flow, CFD solutions can be obtained from continuity, momentum, and energy equations with effects of viscosity and thermal conduction as presented in the previous chapter.

High-speed computers deliver numerical solutions by calculating properties at each discrete point throughout the flow field. Grid lines connect each discrete point, or so called grid point, to the other, dividing the domain with grids or meshes. Typical CFD grids are course in the outer region (up to $12 \sim 15 \mathrm{c}$ for a pressure-far-field boundary for a subsonic flow), and they are extremely fine and dense at the near-wall regions as $y^{+} \approx 1 \sim 5$.

Commercial software is utilized for domain grid generation. A meshing technique for the complex and challenging domain of an airfoil with a leading-edge slat is discussed in the next chapter. ANSYS Fluent 12.0 two-dimensional is the solver and the post-processor utilized. In the next section, some of the turbulent-viscous models and their applications are explained. It is most critical to choose an appropriate turbulence model to achieve validation and accurate results, and perhaps the most run time efficiency well. This is because resources and references are not available for all geometry and boundary condition. 


\subsection{Turbulence Models}

The characteristics of turbulent flow are presented by the velocity fluctuation components. Flow mass, momentum and energy are transported by fluctuating velocities mixing these quantities. The low frequency and small-scales of these fluctuations typically make the simulation very difficult and expensive. Instead, the exact solution of the time-averaged governing equations can be found by eliminating the small-scales. Yet, the reduced turbulence equation has additional, unknown variables, and the modified model has to determine these as known terms [26].

Unfortunately, there is no one universal turbulence model that is acceptable and superior for all types of problems. Choosing an appropriate turbulence model depends on the domain of interest in the flow, available simulation time and computational resources, required level of accuracy, and the physics of a specific class of problem. We need to understand the performance and limitations of the different options to decide which model is the best for our application. This section reviews the various turbulence models available and capable of solving flows around an airfoil with unsteady separated flow at high angles-of-attack. Turbulent flows are defined as unsteady.

Fluent provides the following turbulence models:

\section{- Spalart-Allmaras model (One-equation):}

The Spalart-Allmaras (SA) model is a simple model used to solve one transport equation for the turbulence (kinematic eddy) viscosity. This model is able to calculate a local shear layer thickness for the length scale with one-equation. The Spalart-Allmaras model is designed especially for wall-bounded flows and adverse pressure gradients boundary layers in aerospace applications. Wall functions implemented, in Fluent, best fits models with relatively coarse meshes, where accurate computations for turbulent flow are not essential. The Spalart-Allmaras model is relatively 
new, yet it is suitable for many complicated engineering problems. References [26, 27] show details of the modeling for transport equations.

\section{- $\quad \kappa-\varepsilon$ models (Two-equation):}

Two-equation models are the simplest "complete" turbulence models capable of finding solutions to two separate transport equations. In this model, two transport equations determine the turbulent velocity and length scale independently. The standard $\kappa-\varepsilon$ model is a semi-empirical model proposed by Launder and Spalding that is well-known for its reasonable accuracy, economy and strength in wider range of turbulent flows, including heat transfer simulations. The term $\varepsilon$ represents the dissipation rate and $\kappa$ is the turbulence kinetic energy derived from the Navier-Stokes equations. Options in the $\kappa-\varepsilon$ model include:

- Standard model

- Renormalization-group (RNG) model

- Realizable model

\section{- $\quad \kappa-\omega$ models (Two-equation):}

There are two $\kappa-\omega$ models with similar forms of transport equations: the Standard and the Shear-stress Transport (SST) equations for $\kappa$ and $\omega$ (where $\omega$ is the specific dissipation rate). The SST model, compared to the standard $\kappa-\omega$, has a relatively gradual change from the inner to the outer region of the boundary layer, where the $\kappa-\varepsilon$ high-Reynolds-model is used. Another difference in the SST model is that the transport effects of the turbulent shear stress are included in a modified turbulent viscosity equation $[26,28]$. The SST model is a blend of the $\kappa$ - $\omega$ standard model near the wall and the $\kappa-\varepsilon$ model away from the surface. This model is more accurate and satisfies a wider class of flows, such as airfoils, adverse pressure gradients and transonic shock waves.

- Standard model 
- Shear-stress Transport (SST) model

\section{- Transition models:}

- Transition $\kappa-\kappa l-\omega$ model (Three-equation)

The transition $\kappa-\kappa l-\omega$ model is capable of calculating the transition start and predicting the boundary layer development. Laminar to turbulent boundary layer transition can be addressed effectively in this model. Three transport equations are implemented in $\kappa-\kappa l-\omega$ model as an eddyviscosity type where laminar kinetic energy $\kappa l$, turbulent kinetic energy $\kappa$, and the inverse turbulent time scale or the specific dissipation rate $\omega$ are the important parameters.

- Transition SST model (Four-equation)

The transition SST model is similar to the $\kappa-\omega$ SST model with two additional transport equations; one is for transition regime, and one is for intermittency that are based on the momentum thickness Reynolds number. A user-defined empirical-properties option (Langtry and Menter) allows a control on the transition onset and the momentum thickness equation.

\section{- Reynolds Stress models (Five-equation):}

The Reynolds Stress Model (RSM) is the most extensive and complex turbulent model in Fluent, with five transport equations in 2D flows and seven in 3D flows to be solved. RSM solves the transport equations for the Reynolds stresses and the dissipation rate that closes the Reynoldsaverage Navier-Stokes (RANS) equations. RSM accounts for the effects of complex flow like rotational, swirling flow with streamline curvature potentially more precise than any other model. Yet, the closure assumptions, in the exact Reynolds stresses transport equations, play a great role in changing the prediction reliability and its limitations. Pressure-strain and dissipation-rate are two challenging terms that are usually responsible for the accuracy of RSM solutions. The best 
applications for RSM are: highly swirling flow, stress-induced flows and rotating flow ducts with anisotropy in the Reynolds stresses.

- Linear pressure-strain model

- Quadratic pressure-strain model

- Low-Re stress-omega model

\section{- Large Eddy Simulation model with the optional sub-scale models:}

A mixture of different length and time scales are the characteristics of eddies in turbulent flows. The size of the largest eddies have the identical length scales of the mean flow. The dissipation of turbulent kinetic energy is caused by the smallest scales in the flow. Large Eddy Simulation (LES) solves large eddies directly, whereas small eddies are modeled as in RANS. Most of the flow momentum, mass and energy are carried by large-scale eddies, which are influenced by the boundary conditions and geometries. However, small eddies are more likely isotropic and independent of the geometries, hence, they easier for modeling than large-scales.

LES requires a considerably long flow run-time to stabilize the solution, in addition to a significantly finer mesh than ones used for RANS calculations. Therefore, the computational cost, in terms of CPU time and memory usage, is much higher than that for a steady RANS model simulation.

\section{- Detached Eddy Simulation model with RANS models:}

The Detached Eddy Simulation or DES model applies RANS models at the boundary layer region and LES in the separated field (i.e., away from the boundary layer). Normally, unsteady large-scales dominate the LES regions where the bulk of the turbulence exists. The DES model switches from LES-like at the separated region to the RANS model at the near-wall, or the boundary layer region. The RANS model can be selected by Fluent users from the following: 
- Spalart-Allmaras model

- Realizable $\kappa-\omega$ model

- SST $\kappa-\omega$ model

The DES models are frequently referred to as the hybrid RANS/LES that combines these two models for the high Reynolds external flow applications. The computational costs are less than LES on CPU resources but still greater than RANS. In the next section, we discuss a new DES option called Delayed Detached Eddy Simulation. By far, this model is the best suited for the domain of our interest: that is, the slatted airfoil for model validations.

\subsection{Delayed Detached Eddy Simulation}

As we explained, the DES model is known for a hybrid of the turbulent RANS model in thin boundary layers. This method requires "squished" meshes on the surface, and the LES mode in separation regions with grid cells mostly square or isotropic. In 2006, Spalart et al [29] introduced Delayed Detached Eddy Simulation (DDES), a modified version of the DES model that was initially presented in 1997. Although the DES formulation has been proven, its application to the high Reynolds number separated flows, often shows false behavior for shallow separations and thick boundary layers. However, DDES is more appropriate for the natural applications [29-31].

Menter and Kuntz [32] introduced $F_{2}$ and $F_{1}$ functions to the RANS two-equations, SST to determine the boundary layer solution for DES and avoid switching to LES. The suggested DDES is using the derivative of the same functions but not limiting it to SST models. This model, generally, can be implemented on all types of problems involving eddy viscosity. Blending functions $F_{2}$ or $F_{1}$ will "preserve RANS mode" or "delay LES function" in the boundary layer [32]. The $F$ functions are designed to become 1 in the boundary layer and drop to 0 immediately at the outer region. 
A three-element airfoil is tested in reference [29] to demonstrate one of the most complex geometries (e.g., see Figure 6) in DDES. This flow has strong pressure gradients, boundary layer and wake interactions with large low-speed regions. It requires high quality meshing of structured grids in different areas. The three-dimensional grid has $250,000 x-y$ points and $31 z$ points (i.e., spanwise) with $z=0.06 c$ and chord Reynolds number of $1.8 \times 10^{6}$. The time step size, $\Delta t$, is equal to $2.2 \times 10^{-5} \mathrm{c} / U_{\infty}$.

Figure 17 shows the boundary layer thickness $\left(U / U_{\infty}\right)$ and eddy viscosity $\left(\mu / \mu_{t}\right)$ profiles close to the trailing-edge of the main element. Note that the DES eddy viscosity is reduced drastically compared to RANS model and how DDES has recovered this parameter in the wall vicinity. At $d / c$ $=0.045(d$ is the distance from the wall) the slat wake is narrower in DDES than the other two models where the boundary layers of the slat and main airfoil merge together. Overall, the DDES provides reasonable results for such a complex geometry. This result has also been confirmed by the author for the two-dimensional problem of slatted airfoil and will be addressed in upcoming sections.
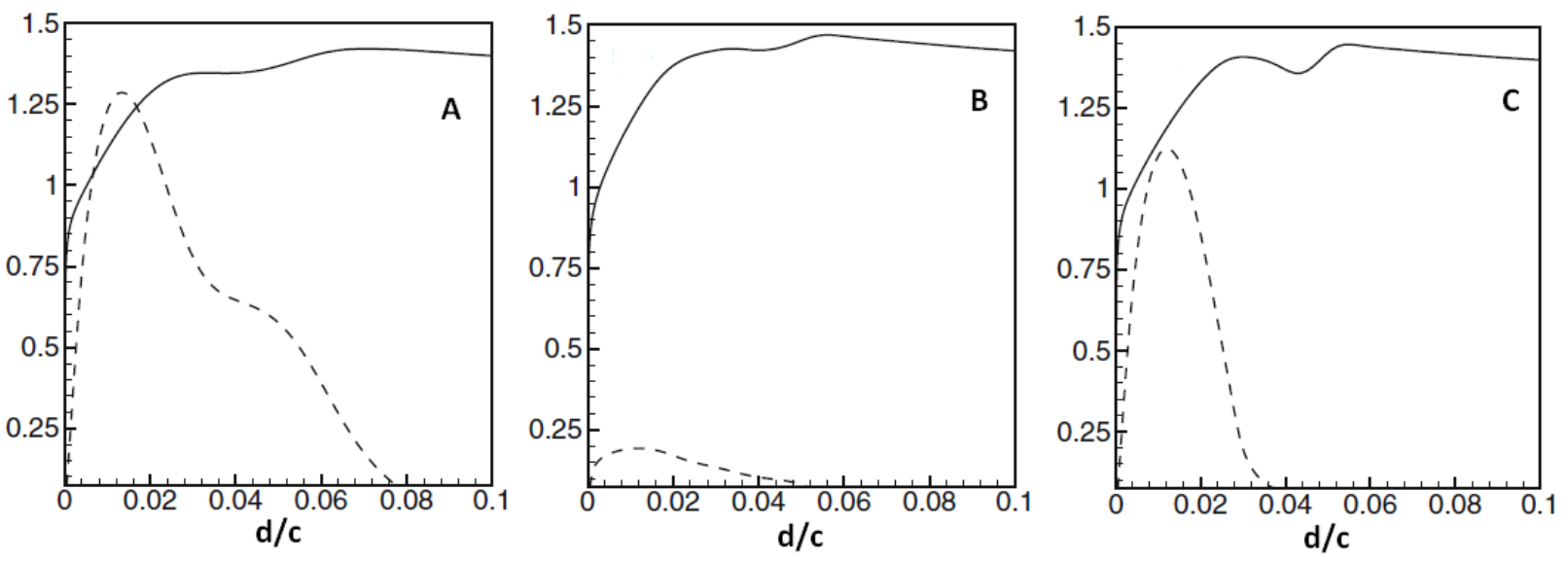

Figure 17. Boundary layer distribution at $x / c=0.95$ over the main airfoil, (A) RANS, (B) DES, (C) DDES, _ $U / U_{\infty},---\mu / \mu_{t}[30]$ 


\section{CHAPTER 4}

\section{MODEL VALIDATION}

\subsection{Grid Generation}

Approximations are used to solve the governing differential equations of fluid mechanics. This is accomplished by converting partial derivatives to a finite difference form. These finite difference equations, also known as approximate algebraic equations, are solved at discrete points inside the domain of interest. Hence, a set of grid points need to be defined within the boundaries of the domain [33].

Generally, a domain has a rectangular shape where grid points are assigned to each grid line, making grid points easier to be identified. This domain is called a structured grid or a rectangular mesh (if 2D). On the other hand, grid points can be generated in a less orderly manner. This domain is known as an unstructured grid or a triangular mesh (if 2D). In order to obtain more accurate results in the boundary layer, a dense or fine mesh is necessary to calculate viscous shear layer behavior $\left(y^{+} \approx 1\right)$. However, a fine mesh throughout the domain causes an increase in grid points, and eventually the CPU time. It is recommended to tailor the grid size near the wall to add precision. This process is referred to as clustering, which is easier to implement by using structured mesh [34, 35]. 
A commercial grid-generator ANSYS Gambit 2.4 is used to mesh our domain. Figure 18 presents the single-element NACA $64_{1-2} 212$ domain as it extends 20 chord lengths downstream and

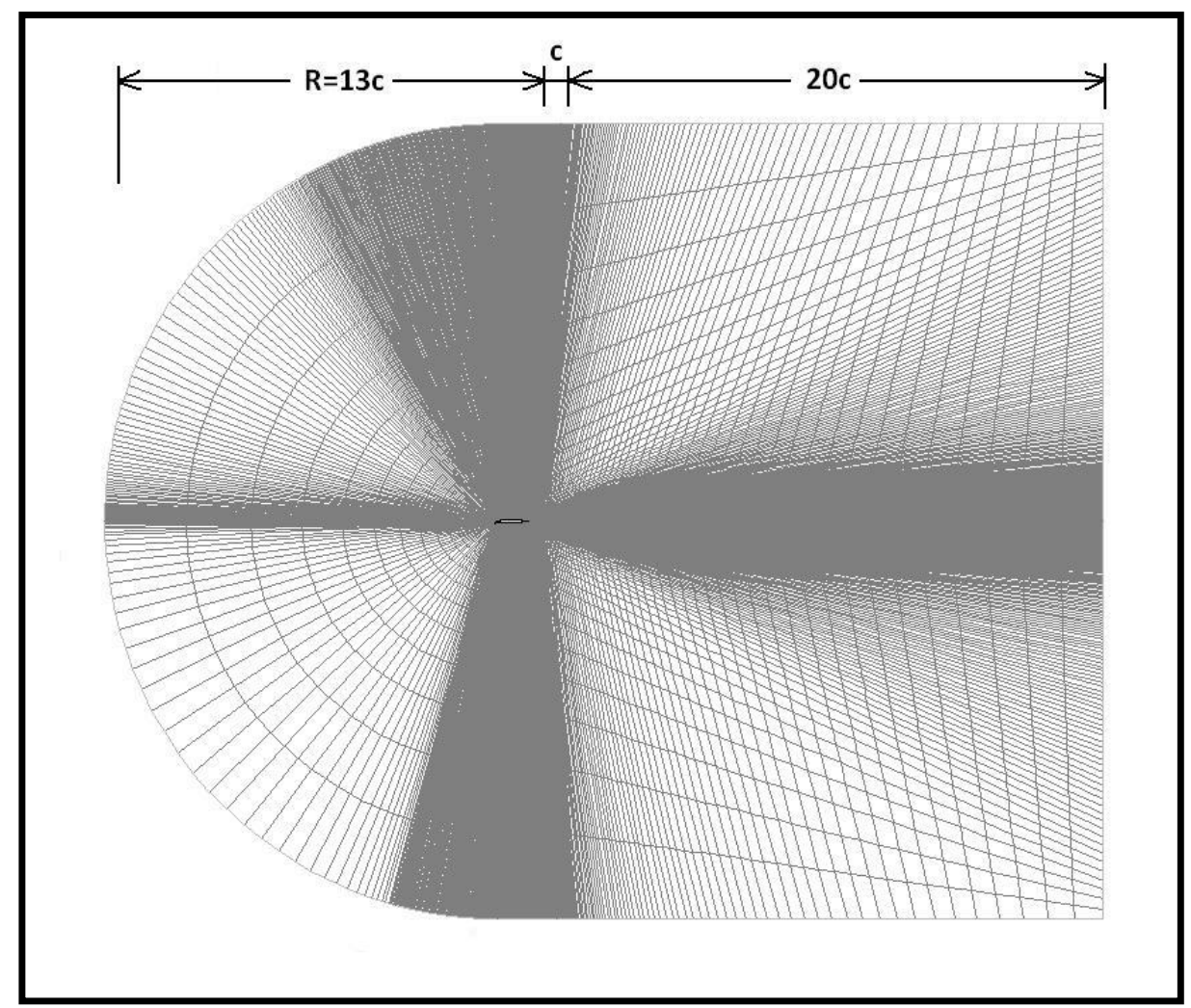

Figure 18. Single-element airfoil domain size and clustering

13 chord lengths to the sides and upstream. A two-dimensional (2D) structured C-grid domain is applied in $x-y$ direction, which consists of 187,000 grid points. To increase the grid points at the surface of the wall, highly skewed mesh generation is inevitable. The mesh maximum aspect ratio is approximately 3,000. The domain boundary is set as a pressure-far-field, and the surface of the airfoil is set as a wall. 16 different zones divide the whole domain by interior lines. Maximum $y^{+}$ for upper surface is held below 1.5 and for lower surface 3.0.

A similar C-grid domain is used for a slatted NACA $64_{1}-212$ airfoil (see Figure 19). The complex geometry at the leading-edge requires the meshing technique to generate a structured grid in the entire region. Effective meshing is implemented in the domain by using 151,000 grid points 
with a maximum aspect ratio of approximately 3,000. Maximum $y^{+}$on the leading-edge slat is 2.5 for the lower surface, and $y^{+} \leq 1$ for the main airfoil.

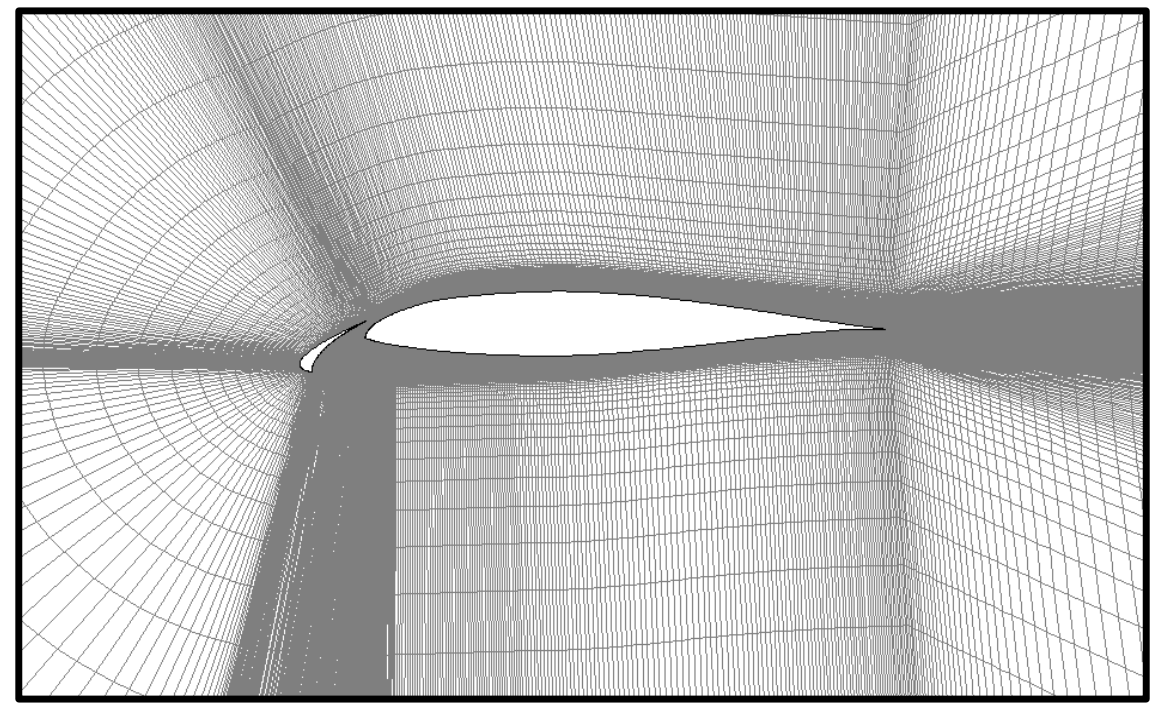

Figure 19. Grids over slatted NACA $64_{1}-212$

A zoom image of the plain and slatted airfoils is shown in Figure 20. Notice the complexity in the leading-edge slat and the sharp leading-edge of the main airfoil. Although grids are skewed at this area, the maximum grid squish is equal to the single-element airfoil one. Coordinates of the NACA 64-212 airfoil section with a leading-edge slat are available in reference [11].

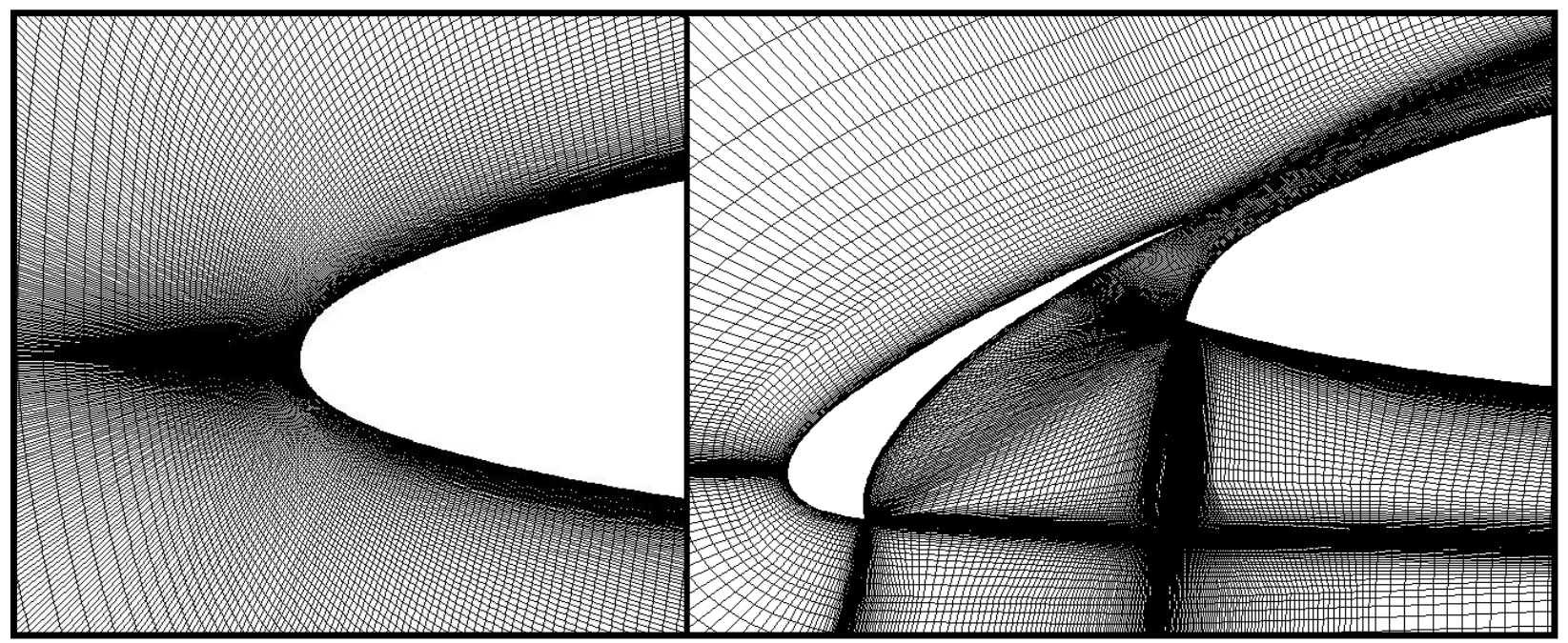

Figure 20. Zoom around the leading-edge 


\subsection{Turbulence Model}

Although the accuracy of simulation models improve each day, not many studies present a best single CFD code to show the behavior of an airfoil at high angles-of-attack or at stall with massive flow separation. Although a few attempts have been made [36], no practical airfoil geometry has been tested. Therefore, the industrial design applications using CFD on high-lift multi-element airfoils have not been established yet.

In order to evaluate new conceptual design concepts with CFD simulation, we need to examine the most suited turbulence model. It is necessary for the code to be applicable to a practical airfoil geometry, including a leading-edge slat. Meanwhile, it is possible to reduce the validation run-time by beginning with a simple geometry and then advance to the complicated configurations.

CFD testing on the NACA 4-series is popular for its insensitive characteristics to the geometry roughness. These airfoils are known for their large, round leading-edge and thick, highcambered shape. Initial validation results indicate that both models $\kappa-\omega$ SST and RSM predict the aerodynamic parameters of NACA 4412 reasonably well, when compared with the experimental data [37]. These models demonstrate the stall at 20 degrees, supporting experimental results. However, the RSM capability to simulate steady flow is an advantage, giving the highest precision (less than $1 \%$ difference) at zero angle-of-attack for $C_{l}$.

The next step in this work is to verify the model on different single-element and multielement airfoils. The NACA $64_{1}-212$ is chosen for final model validation given its practicality and complex geometry at the leading-edge. A good outcome indicates a positive application for industrial purposes of that model. Figures 3 and 4 and table 1 show experimental lift and moment coefficients [11, 38]. No drag effect is detected in reference [11] for NACA $64_{1}-212$ with the leading-edge slat, whereas reference [37] measured a single-element drag and moment coefficients 
at the aerodynamic center. Table 3 displays turbulence model dependency on total mesh size and angles-of-attack. Tested models in this table were chosen based on trends noted in the previous NACA 4412 results.

TABLE 3

TURBULENCE MODEL VALIDATION ON SINGLE-ELEMENT NACA 64 1 -212

\begin{tabular}{|c|c|c|c|c|c|c|c|c|c|c|}
\hline \multirow{2}{*}{\multicolumn{2}{|c|}{$\begin{array}{c}\text { Mesh } \\
\text { Size }\end{array}$}} & \multirow{2}{*}{$\mathrm{AOA}$} & \multirow{2}{*}{ Run time } & \multirow{2}{*}{$y^{+}$} & \multirow{2}{*}{$\mathrm{TI}(\%)$} & \multirow{2}{*}{ Wall Treat } & \multirow{2}{*}{ Vis } & Simul & Exp & \multirow{2}{*}{ B.C. } \\
\hline & & & & & & & & $\mathrm{Cl}$ & $\mathrm{Cl}$ & \\
\hline \multirow{6}{*}{$\begin{array}{c}\mathrm{SA} \\
1-\mathrm{eq}\end{array}$} & \multirow{3}{*}{$272 \mathrm{k}$} & 0 & $\mathrm{~S}(393)$ & $\leq 1$ & & & & 0.1594 & 0.13 & $\mathrm{Ma}=0.3, \mathrm{MTV}=0.001, \mathrm{TVR}=10$ \\
\hline & & 15 & & & & & & & & \\
\hline & & 20 & & & & & & & & \\
\hline & \multirow{3}{*}{$423 \mathrm{k}$} & 0 & $\mathrm{~S}(508)$ & $\leq 1$ & 0.05 & $\mathrm{~V}$ (no damp) & 3 & 0.1448 & 0.13 & $\mathrm{Ma}=0.3, \mathrm{MTV}=0.0046, \mathrm{TVR}=10$ \\
\hline & & 15 & $\mathrm{U}(0.5 \mathrm{~s})$ & $\leq 1$ & 0.05 & $\mathrm{~V}$ (no damp) & 3 & $0.98-1.03$ & 1.55 & " \\
\hline & & 20 & $\mathrm{U}(0.6 \mathrm{~s})$ & $\leq 1$ & 0.05 & $\mathrm{~V}$ (no damp) & 3 & $0.87-0.99$ & 1.07 & $"$ \\
\hline \multirow{3}{*}{$\begin{array}{l}\text { kw- } \\
\text { SST } \\
2-e q\end{array}$} & \multirow{3}{*}{$184 \mathrm{k}$} & 0 & & & & & & & & \\
\hline & & 15 & $\mathrm{U}(1.4 \mathrm{~s})$ & $\leq 3$ & 0.05 & - & 3 & $1.29-1.33$ & 1.55 & $\mathrm{Ma}=0.3, \mathrm{TVR}=10$ \\
\hline & & 20 & $\mathrm{U}(0.23 \mathrm{~s})$ & $\leq 3$ & 0.05 & - & 3 & $0.98-1.22$ & 1.07 & $\mathrm{Ma}=0.3, \mathrm{TVR}=10$ \\
\hline \multirow{3}{*}{$\begin{array}{c}\text { k-kl-w } \\
3-e q\end{array}$} & \multirow{3}{*}{$423 \mathrm{k}$} & 0 & & & & & & & & \\
\hline & & 15 & $\mathrm{U}(0.15 \mathrm{~s})$ & $\leq 1$ & - & & 3 & 0.864 & 1.55 & $\mathrm{k}-\mathrm{kl}$ - $\mathrm{w}$ const $=1$ \\
\hline & & 20 & $\mathrm{U}(0.14 \mathrm{~s})$ & $\leq 1$ & - & & 3 & 0.89 & 1.07 & $\mathrm{k}-\mathrm{kl}$ - $\mathrm{w}$ const $=1$ \\
\hline \multirow{10}{*}{$\begin{array}{c}\text { RANS } \\
5 \text {-eq }\end{array}$} & \multirow{5}{*}{$184 \mathrm{k}$} & 0 & $\mathrm{~S}(832)$ & $\leq 1$ & 0.05 & w/enh & 3 & 0.161 & 0.13 & $\mathrm{k}-\mathrm{e}$ const $=1$ \\
\hline & & \multirow{3}{*}{15} & $\mathrm{U}(0.7 \mathrm{~s})$ & $\leq 1$ & 0.05 & Stand & 3 & 1.09-1.11 & 1.55 & No k-e \\
\hline & & & $\mathrm{U}(0.6 \mathrm{~s})$ & $\leq 1$ & 0.05 & w/enh & 3 & 1.07-1.11 & 1.55 & No k-e (with k-e diverge) \\
\hline & & & $\mathrm{U}(0.6 \mathrm{~s})$ & $\leq 1$ & 0.05 & w/enh & 2 & $0.8-0.9$ & 1.55 & No k-e (with k-e diverge) \\
\hline & & 20 & & & & & & & & \\
\hline & \multirow{2}{*}{$272 \mathrm{k}$} & \multirow{2}{*}{0} & $S(670)$ & $\leq 1$ & - & w/enh & 3 & 0.145 & 0.13 & $\mathrm{k}-\mathrm{e}$ const $=1$ \\
\hline & & & $\mathrm{S}(859)$ & $\leq 1$ & 1.00 & w/enh & 3 & 0.195 & 0.13 & TVR=10 \\
\hline & \multirow{3}{*}{$423 \mathrm{k}$} & 0 & S (1020) & $\leq 1$ & - & Stand & 3 & 0.144 & 0.13 & $\mathrm{k}-\mathrm{e}$ const $=1$ \\
\hline & & 15 & & & & & & & & \\
\hline & & 20 & & & & & & & & \\
\hline
\end{tabular}

Notes: Mesh sizes are equivalent for grid points (e.g. $272 \mathrm{k}=272,000$ ); Run-time, S=Steady (iteration number), $\mathrm{U}=\mathrm{Unsteady}$ (Test run-time until the result is stabilized in second); $\mathrm{y}+=$ the maximum value; TI=Turbulence Intensity; Wall treatment, V=Vorticity based, w/enh=Enhanced wall treatment, Stand=Standard; Vis=Viscosity, Sutherland law 2 or 3 coefficients used; B.C., $\mathrm{Ma}=$ Mach number, TVR=Turbulent Viscosity Ratio, MTV=Modified Turbulent Viscosity. 
Note that at the primary stage of code validation, there is some inconsistency in simulation boundary conditions and the actual wind tunnel test's Mach number. Due to limitations of the wind tunnel, the Reynolds number of $6.0 \times 10^{6}$ is obtained by pressurizing the tunnel (that is twoatmospheric-pressure). The initial Mach number was incorrectly increased to 0.3 to reach the test Reynolds number at one-atmospheric-pressure. However, table 3 results give a relatively accurate comparison between the different models.

The SA model for steady flow at zero angle-of-attack has the best compound solution for the shortest computational time and $C_{l}$ prediction. For massive, separated, unsteady flows, at high angles-of-attack, the $\kappa-\omega$ SST is the most consistent and accurate model. In spite of encouraging results for unsteady flow, the $\kappa$ - $\omega$ SST model solution diverges in the steady flow regime. The RMS 5-equation model shows the highest accuracy for stable flow but not for unsteady flows. All the models are nearly independent of the mesh size, as long as the $y^{+}$is kept below or about 1 .

Further investigations have found that the DDES model can be applied to two-dimensional grids as well. Meshing refinement and configuration optimization are implemented using XFoil to generate additional coordinate points. By far, in the DDES with an optional RANS model $\kappa$ - $\omega$, the SST results are the most promising in terms of less CPU time and the highest accuracy for unsteady flows. CPU time consumed in the DDES model is approximately one third to half of the 3-equation $\kappa-\kappa l-\omega$ model for 150,000 grids on a single-element airfoil.

Figures 21 and 22 show the single NACA $64_{1}-212$ airfoil aerodynamic data gained from the DDES model compared to the experimental results. Note that $C_{l \max }$ matches at 15 degrees of angleof-attack within $6 \%$, and, even after stall at 20 degrees, the difference is held by less than $10 \%$. 


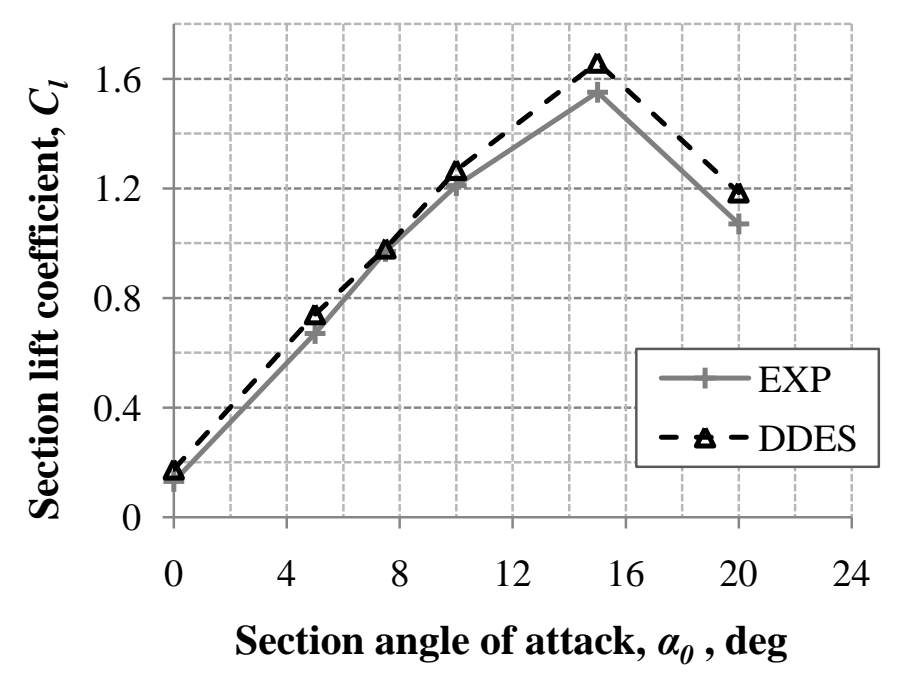

Figure 22. NACA $64_{1}$-212 single-element lift coefficient at $\operatorname{Re}=6.0 \times 10^{6}(\mathrm{EXP}:$ experiment data [37] $)$

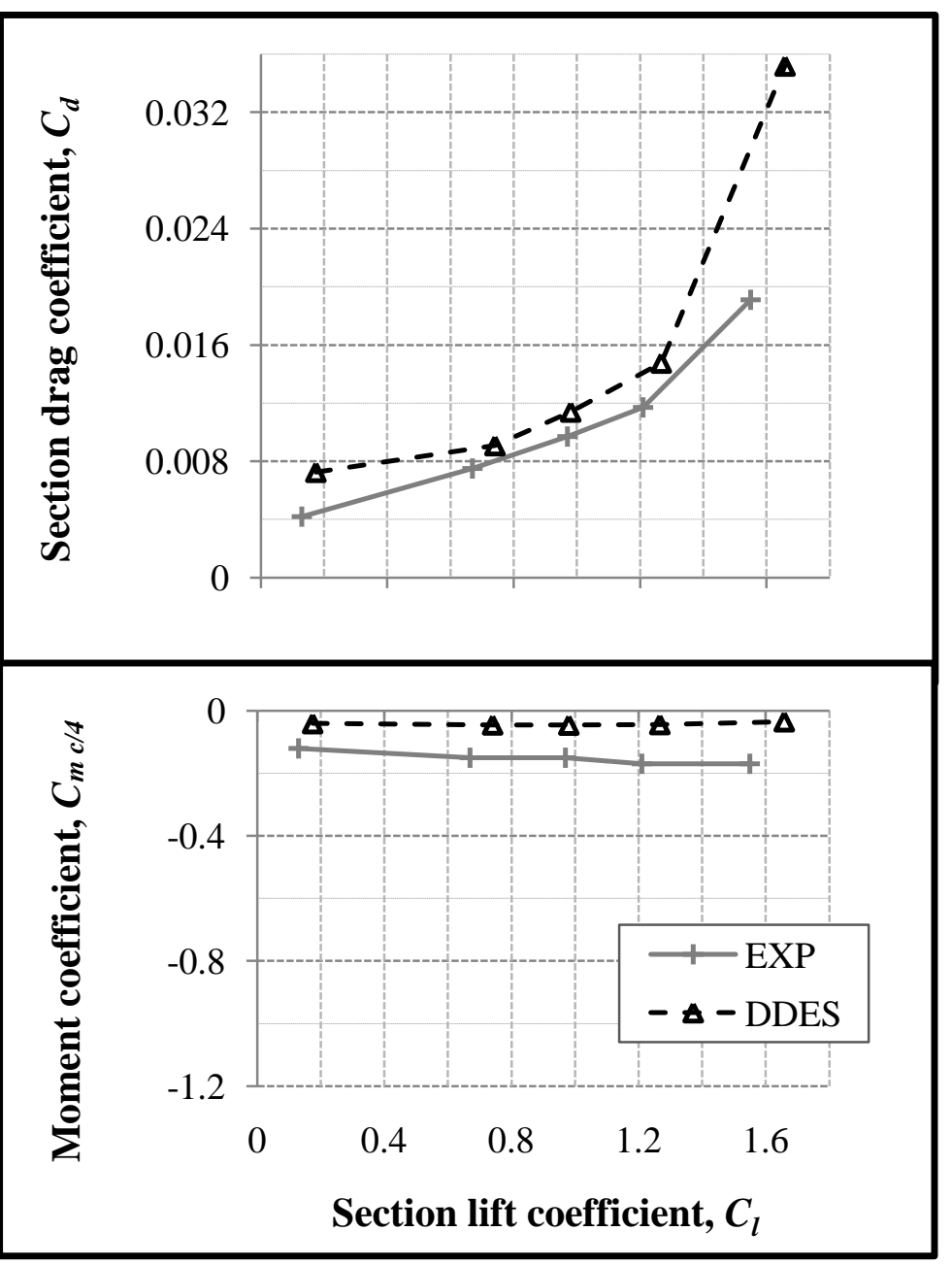

Figure 21. NACA 641-212 single-element drag polar and a quarter chord pitching moment coefficient at $\operatorname{Re}=6.0 \times 10^{6}(\mathrm{EXP}:$ experiment data [37]) 
The drag coefficient prediction is the highest obstacle in CFD simulations, though DDES obtained reasonable data, especially up to point of $C_{l \max }$. Time step size $\Delta t$ was set to $1.0 \times 10^{-4}$ for less computational time. Flow at zero angle-of-attack observes unsteady flow, since steady flow is not available on DDES. Figure 23 presents lift and moment coefficients for the NACA $64_{1}-212$ with a leading-edge slat. 184,000 grid points are used for the slatted airfoil with a maximum $y^{+}$less than 5 .

There is an outstanding agreement in the linear segment of the lift coefficient up until 15 degrees of angle-of-attack. Contrary to experimental data, as angle-of-attack increases the slope remains nearly constant before 25 degrees with an early stall at $29^{\circ}$. One can speculate that flow

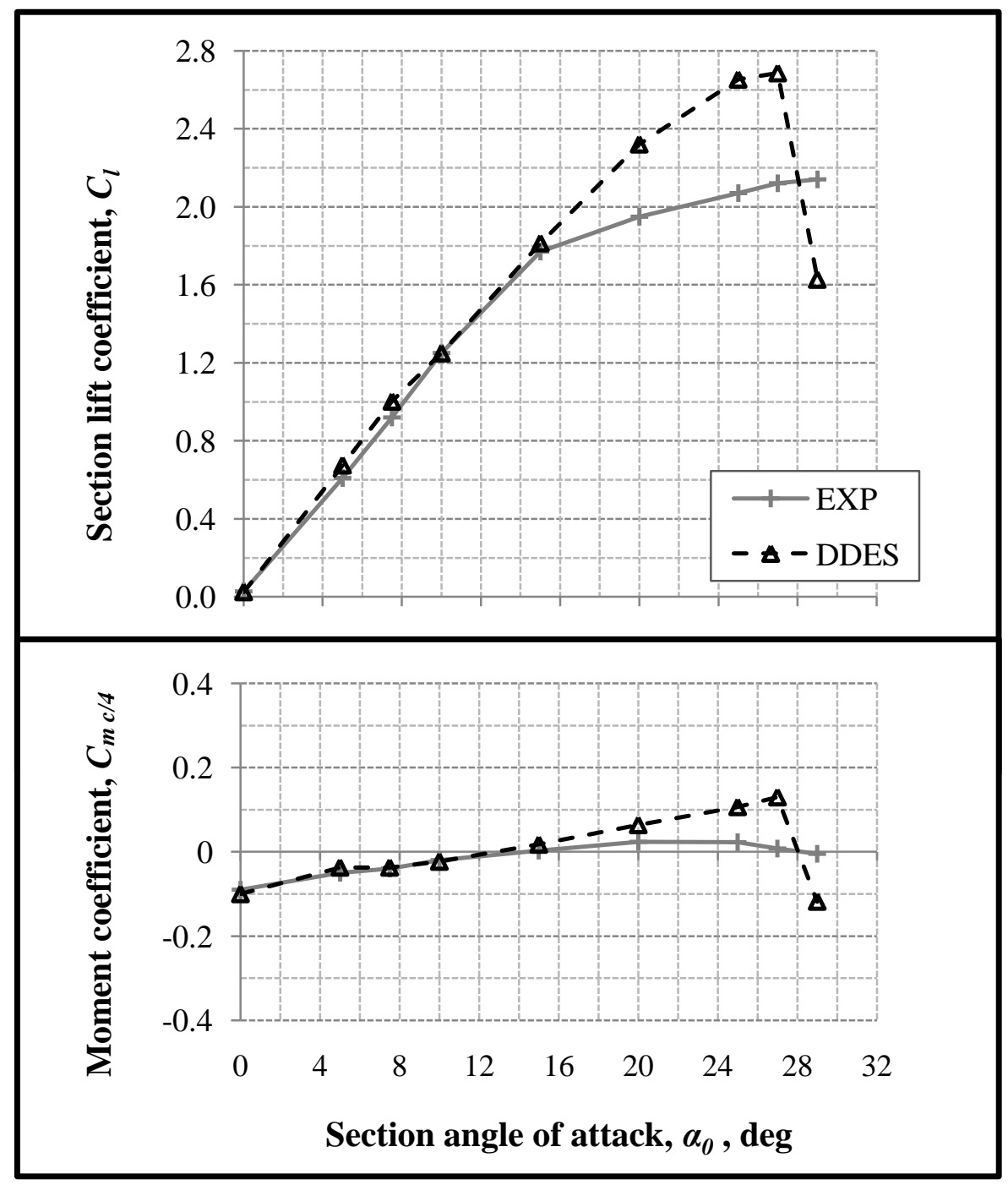

Figure 23. NACA $64_{1}-212$ with the leading-edge slat lift and moment coefficients at $\operatorname{Re}=6.0 \times 10^{6}(\mathrm{EXP}$ : experiment data [11]) 
condition of the simulation and experimental results are similar but not the same. For example, although both tests are 2D, the flow in spanwise direction exists in an experimental finite wing span. The $z$-direction mass flow and vorticity magnitude intensify at the higher angles-of-attack. Meanwhile, flow simulation is a discrete process, unlike empirical continuous data acquisition. CFD data are obtained from an instantaneous boundary condition (e.g., angle-of-attack), whereas experimental angles-of-attack increases gradually in the same test run. Note that a flow separation is a continuous process which usually grows from the trailing-edge as the adverse pressure gradient increases along with the angle-of-attack. The pitching moment coefficient shows similar results.

Boundary conditions for both configurations are set as: $M_{\infty}=0.1325, P_{\infty}=202,650 \mathrm{~Pa}$ or 2.0 atm which gives $\operatorname{Re}=6.0 \times 10^{6}$, a turbulence intensity of $0.05 \%$, the turbulent viscosity ratio as 1 , and this viscosity is obtained using the three-coefficient-method of Sutherland law.

Turbulence model validation is the most important and time consuming process because of the lack of appropriate literature and resources. Figure 24 shows model mesh size dependency on two different domains, 400,000 and 180,000 grid points. Consistent static pressure contours explain

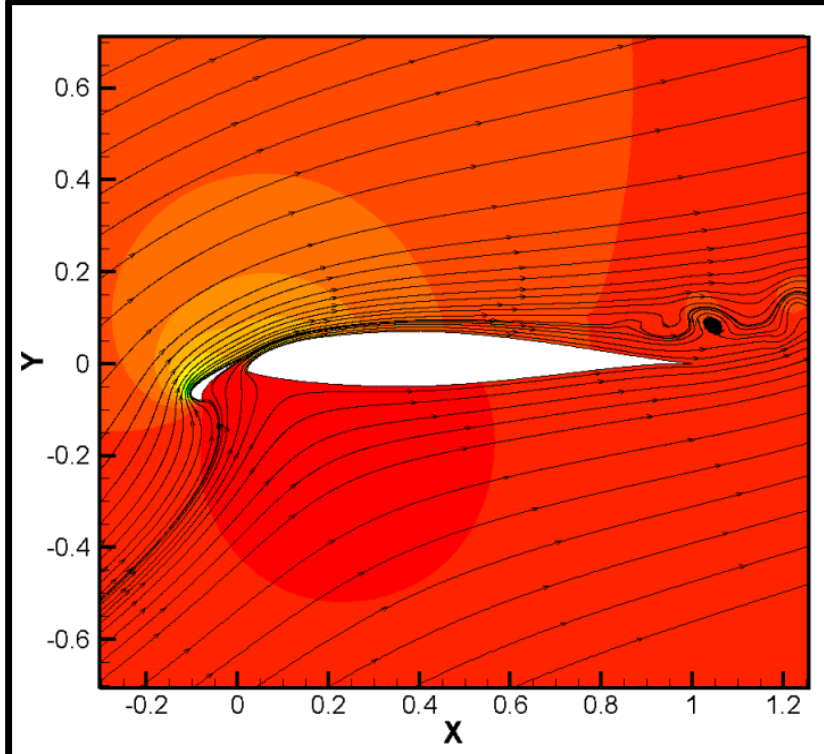

$400 \mathrm{k}$, Run time $=0.4 \mathrm{~s}, C_{1}=2.68$

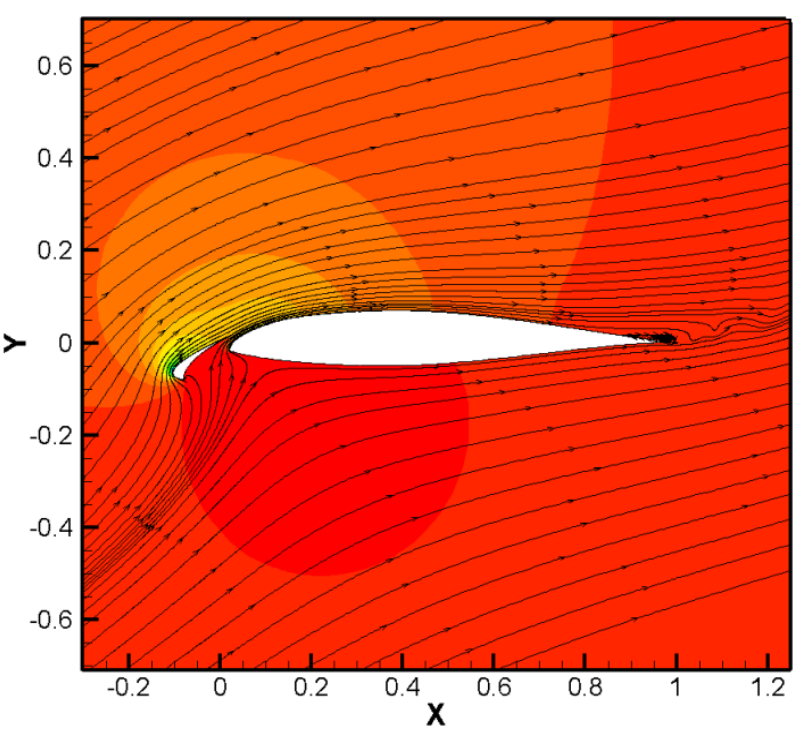

$180 \mathrm{k}$, Run time $=0.4 \mathrm{~s}, C_{1}=\mathbf{2 . 6 3}$

Figure 24. Mesh size dependency on a pressure contour with streamlines at $\mathrm{AOA}=25^{\circ}$ 
the $C_{l}$ small difference between the two models. However, one may be concerned that the flow separation regions are slightly different, whereas, at a smaller mesh size, the separation occurs at the surface close to the trailing-edge.

Time step size dependency is also examined for three different time increments, as $\Delta t=$ $1.0 \times 10^{-4}, 1.0 \times 10^{-5}$, and $1.0 \times 10^{-6}$. No significant change is observed in these results. Two time steps sizes are compared in Figure 25, considering the static pressure contour for a single-element airfoil at $20^{\circ}$. The flow is entirely separated at 0.4 seconds of run-time (Figure 25 right). This figure also shows how run-time influences the flow development. At an early stage, flow is not separated at 0.2 seconds. In most cases, it takes 0.3 to $0.5 \mathrm{~s}$ for the flow to be fully settled or developed. At $20^{\circ}, C_{l}$ drops to 1.00 in $0.47 \mathrm{~s}$ when $\Delta t=1.0 \times 10^{-4}, 0.39 \mathrm{~s}$ at $\Delta t=1.0 \times 10^{-5}$, and $0.34 \mathrm{~s}$ at $\Delta t=1.0 \times 10^{-6}$.

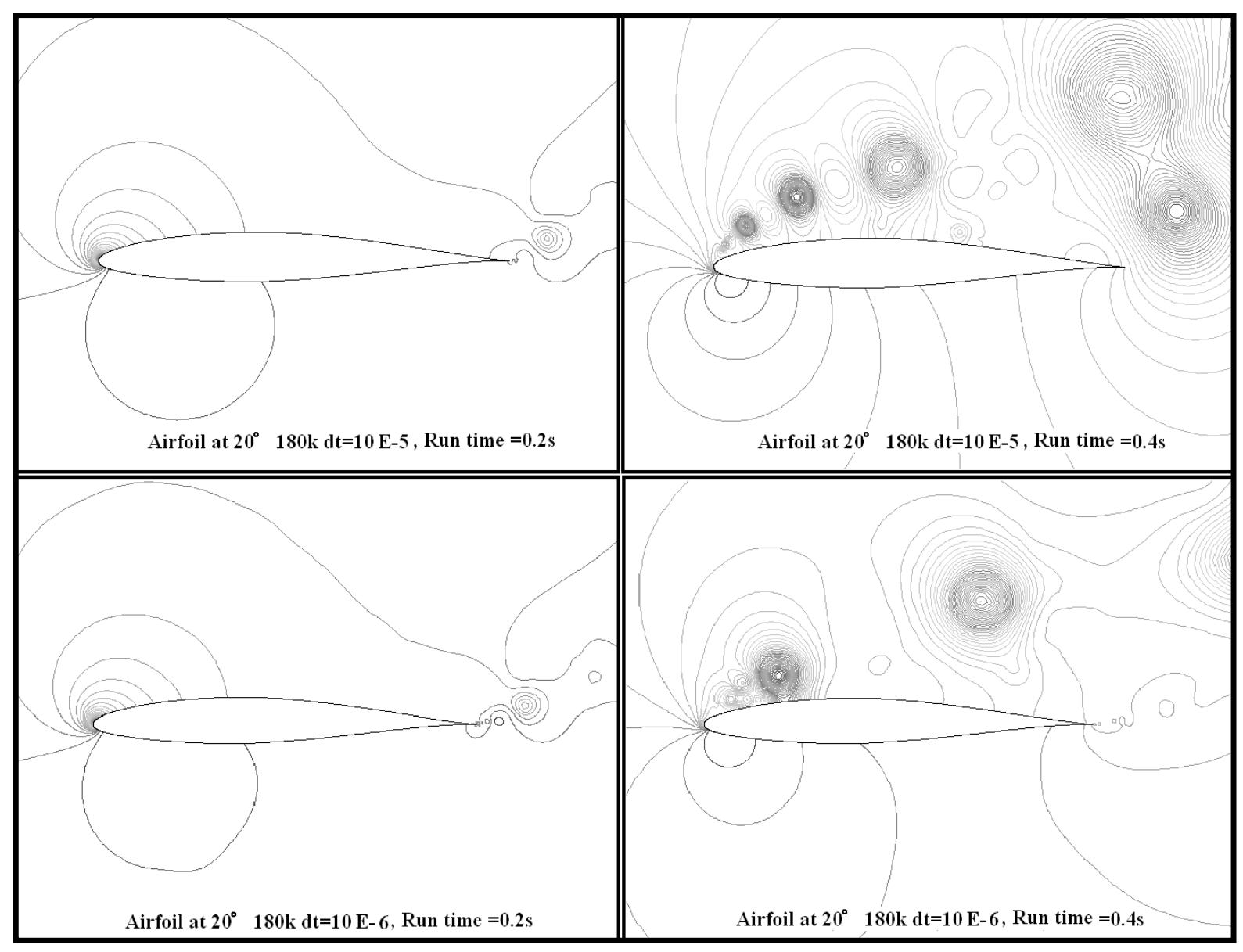

Figure 25. Time step size dependency on a static pressure contour at stall 


\section{CHAPTER 5}

\section{RESULTS}

For each model, several factors, at different possible values or levels, need to be examined. This may lead to infinite simulation time and cost. Using factorial design on Design of Experiment methods, introduced by Montgomery [39], the number of combinations are reduced. In this study, two or three primary factors (e.g., $x$ and $y$ locations), at two levels (+/-) and several angles-of-attack are commonly examined.

A typical device analysis matrix, in this case for the Stall vane, is shown in table 4 . Three factors (i.e., $x, y$ and $\theta$ ) are considered as the most influential. Two levels (+/-) are set to visualize the model dependency for each factor. The model total $C_{l}, C_{d}$ and $C_{m}$ at different angles-of-attack, as well as the flow separation point results, are presented. All tests are implemented at $\operatorname{Re}=6.0 \times 10^{6}$ with identical boundary conditions (including to the validated model).

\subsection{Stall Vane}

Figure 26 shows the model with corresponding $x, y$ and $\theta$ arrangements.

TABLE 4

ANALYSIS MATRIX FOR STALL VANE

\begin{tabular}{cccc||ccc}
\hline \hline model & $\mathbf{x}$ & $\mathbf{y}$ & $\boldsymbol{\theta}$ & & - & + \\
\hline $\mathbf{1}$ & - & - & - & $\mathbf{x}$ & $0.3 \mathrm{c}$ & $0.4 \mathrm{c}$ \\
$\mathbf{a}$ & + & - & - & $\mathbf{y}$ & $0.1 \mathrm{c}$ & $0.15 \mathrm{c}$ \\
$\mathbf{b}$ & - & + & - & $\boldsymbol{\theta}$ & $0 \mathrm{deg}$ & $10 \mathrm{deg}$ \\
$\mathbf{a b}$ & + & + & - & & & \\
$\mathbf{c}$ & - & - & + & & & \\
$\mathbf{a c}$ & + & - & + & & & \\
$\mathbf{b c}$ & - & + & + & & & \\
$\mathbf{a b c}$ & + & + & + & & & \\
\hline \hline
\end{tabular}




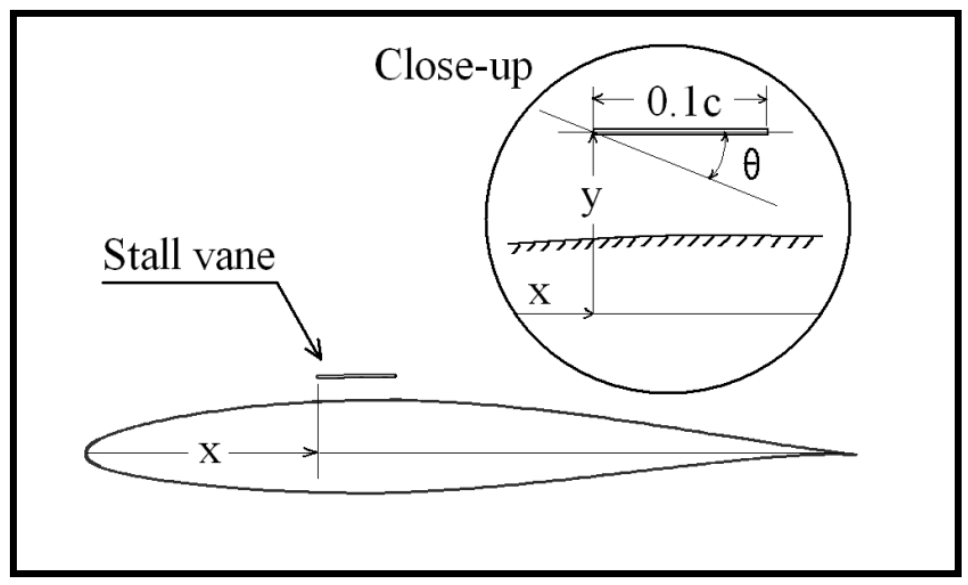

Figure 26. Stall vane design model

Note that the length of the vane is fixed at $10 \%$ of the chord length $(0.1 \mathrm{c})$, similar to the length of a leading-edge slat. To avoid extra test factors, the vane shape is a flat plate with thickness of $0.001 \mathrm{c}$.

A total of eight variations are simulated at four different airfoil angles-of-attack. The section lift coefficient, $C_{l}$, against the angle-of-attack, is presented in Figure 27. The lift-to-drag ratio, drag and pitching moment coefficients against $C_{l}$ are shown in Figure 28. It is evident that at $\theta=10^{\circ}, C_{l}$ has significantly decreased, while drag has increased.

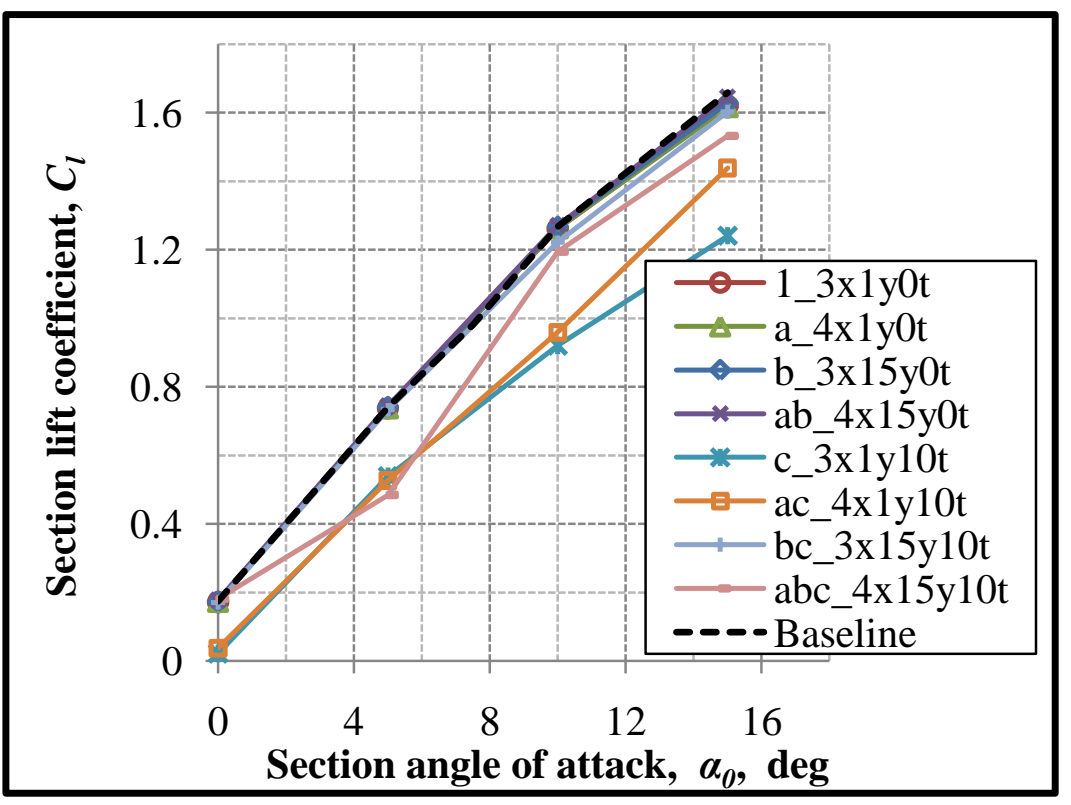

Figure 27. Stall vane lift-to-drag ratio, drag polar and quarter chord moment coefficient at $\operatorname{Re}=6.0 \times 10^{6}$ 


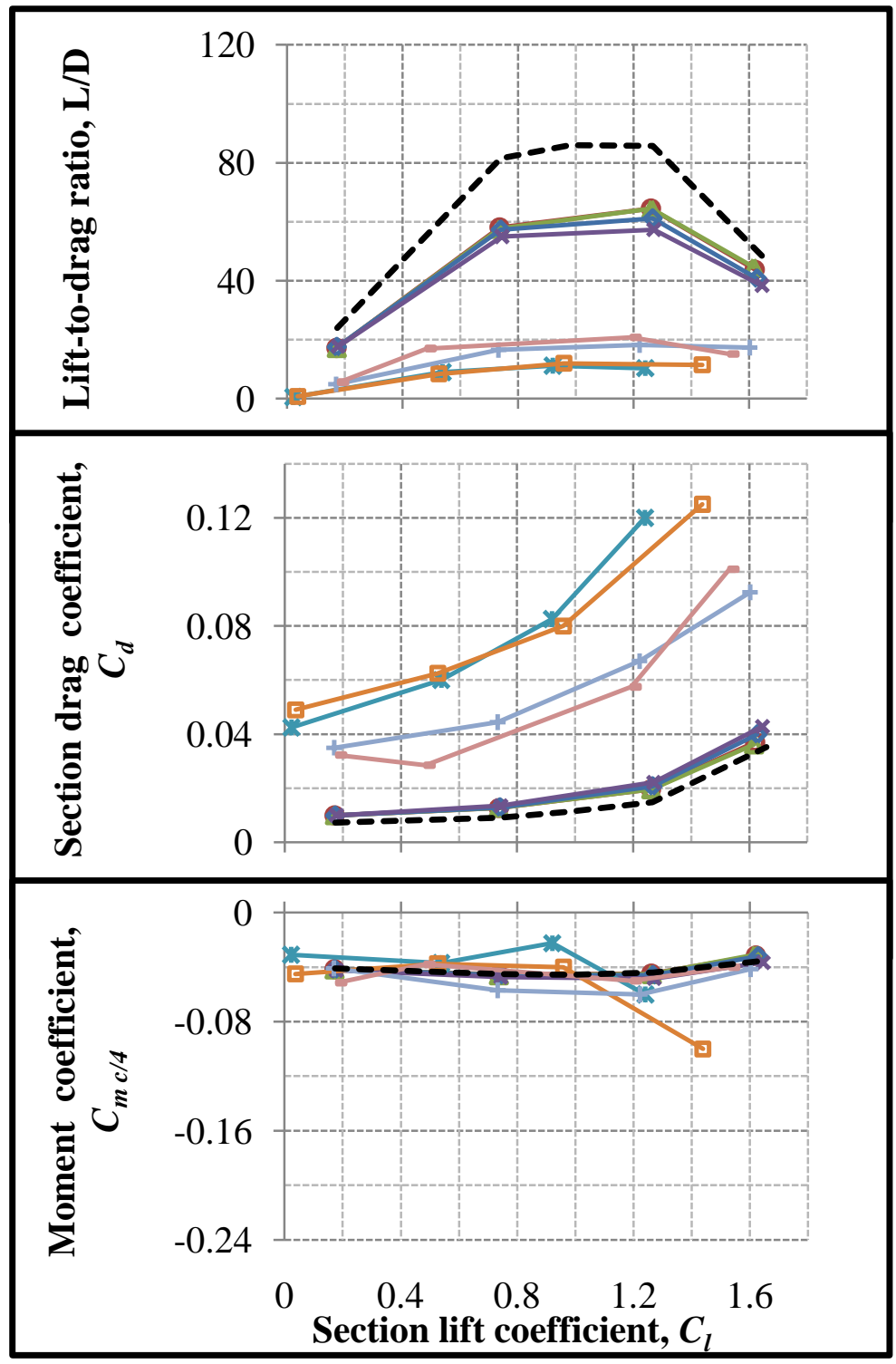

Figure 28. Stall vane section lift coefficient at $\mathrm{Re}=6.0 \times 10^{6}$

Further investigations of streamline contours, as shown in Figures 29-33, show the trailingedge flow separation at $15^{\circ}$ of angle-of-attack. No flow separation has been detected on model (ab) (Figure 32) while the vane is positioned at $(\mathrm{x}, \mathrm{y}, \theta)=\left(0.4 \mathrm{c}, 0.15 \mathrm{c}, 0^{\circ}\right)$. In contrast, separation occurs on the baseline airfoil at $0.86 \mathrm{c}$ (Figure 33). 


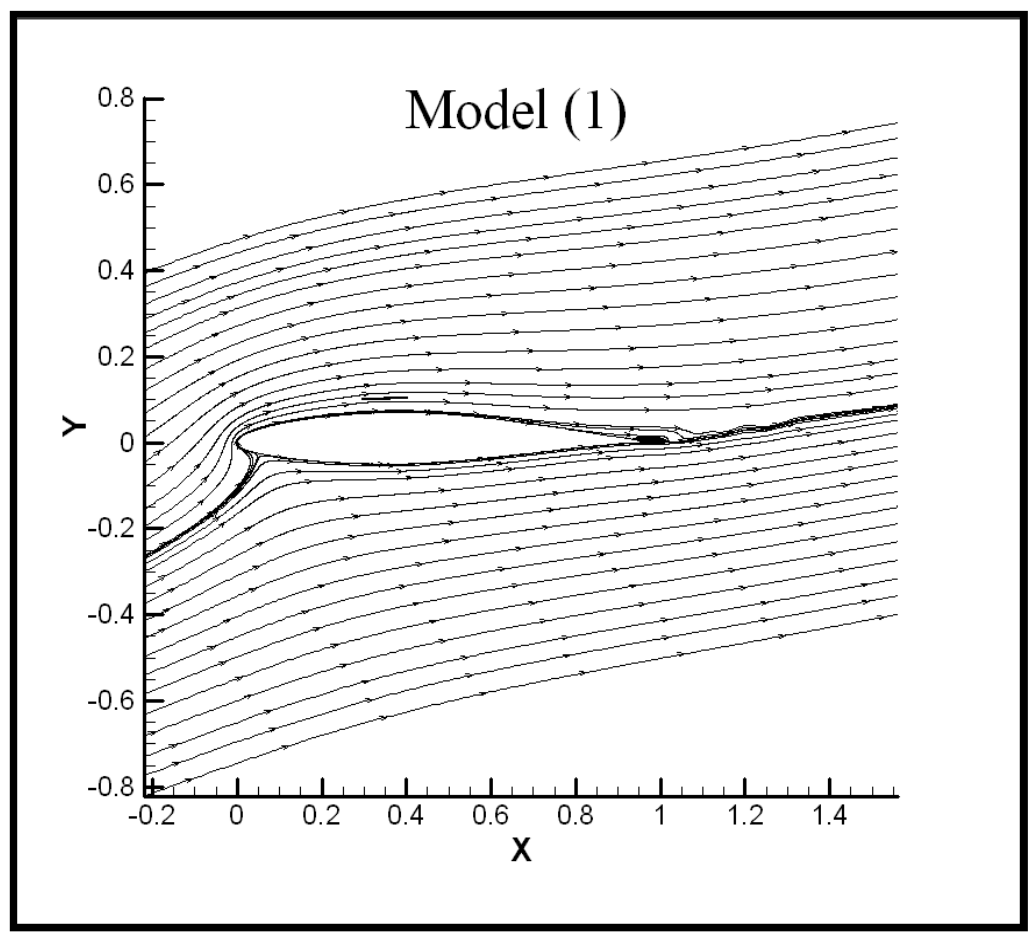

Figure 29. Model (1) at 15 degrees with separation at $0.87 \mathrm{c}$

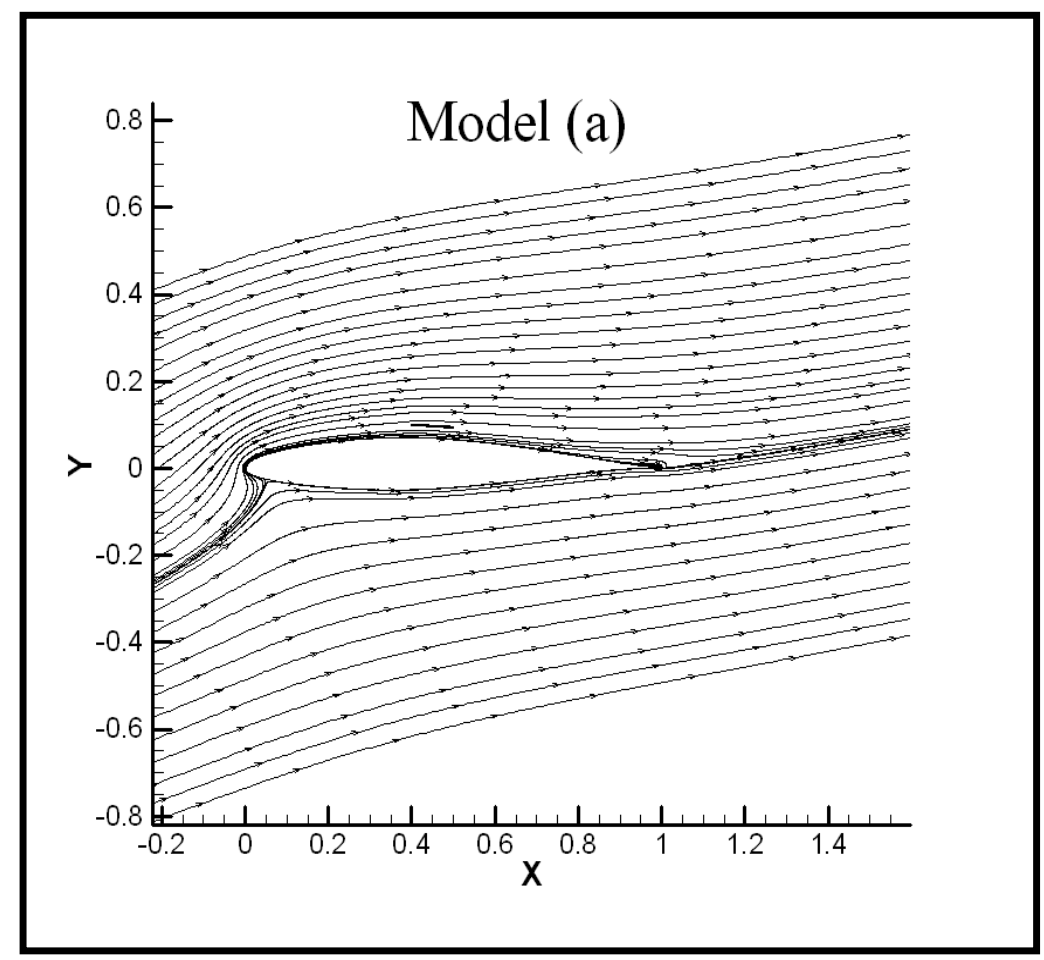

Figure 30. Model (a) at 15 degrees with separation at $0.89 \mathrm{c}$ 


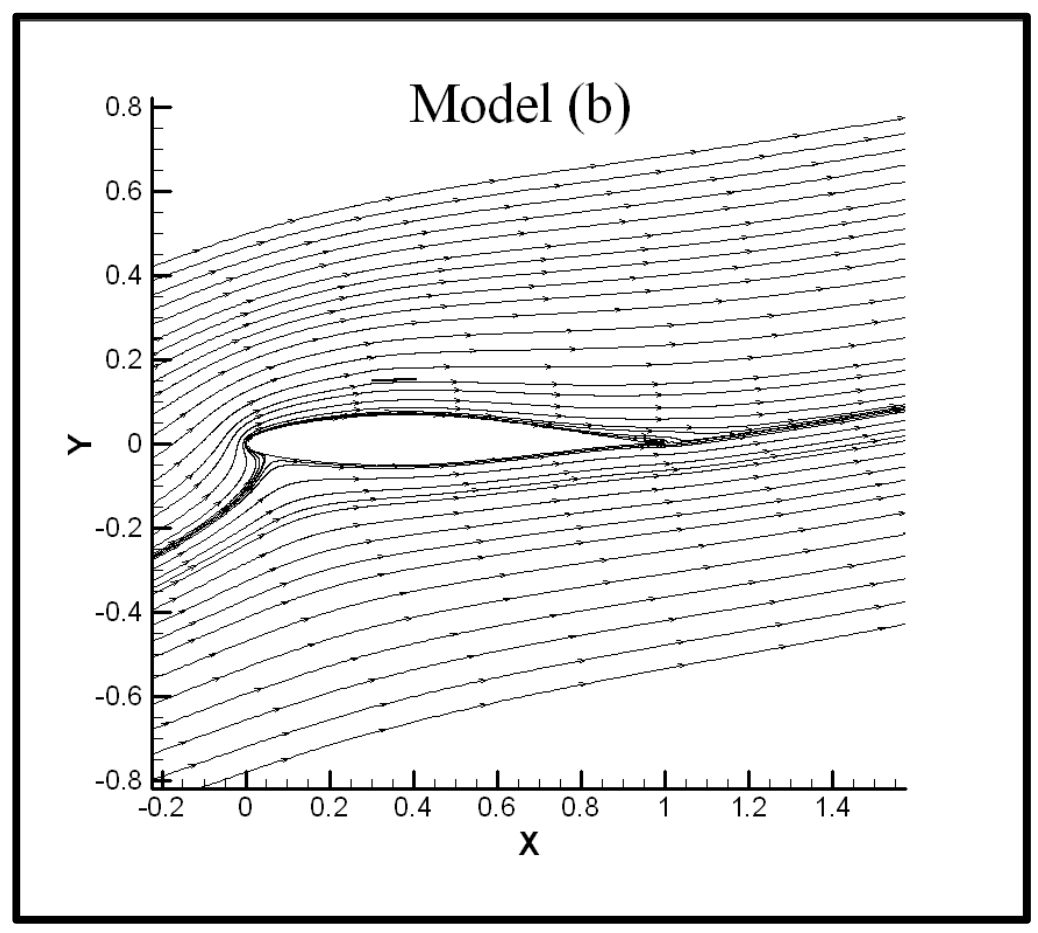

Figure 31. Model (b) at 15 degrees with separation at $0.92 \mathrm{c}$

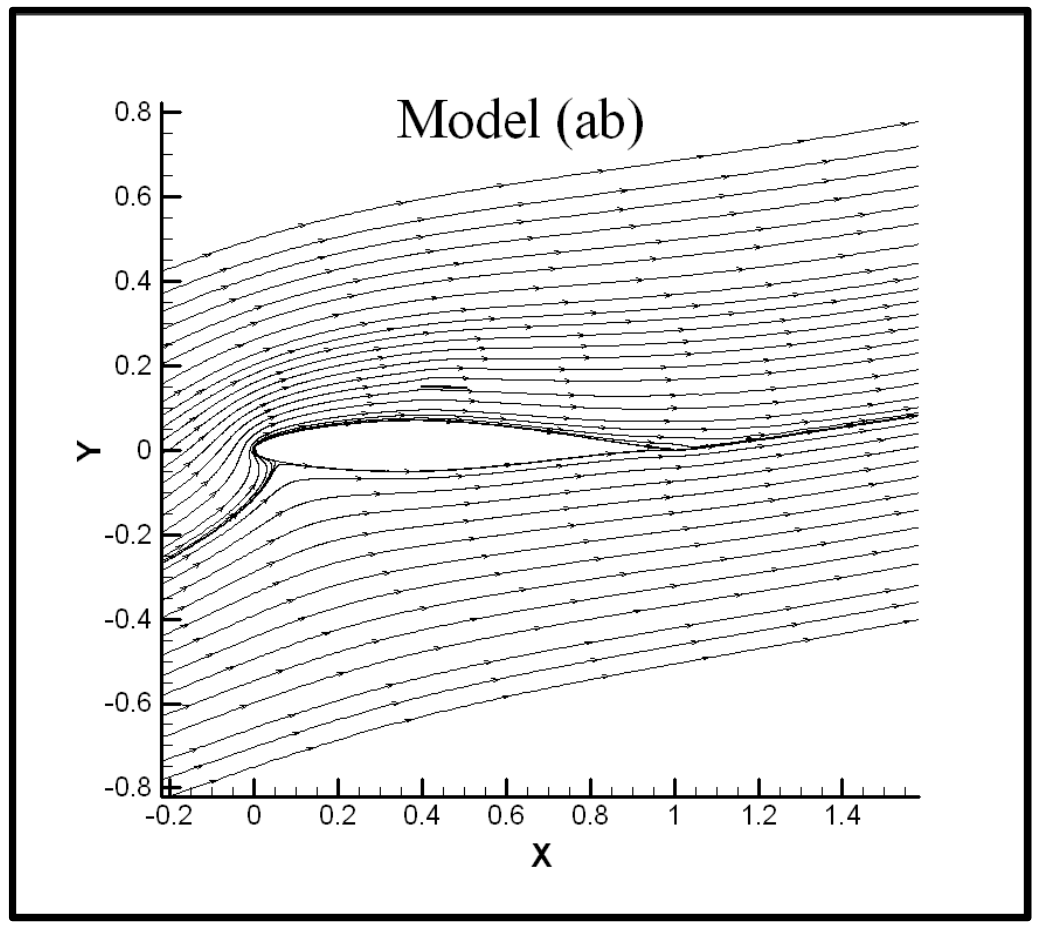

Figure 32. Model (ab) at 15 degrees with no separation 


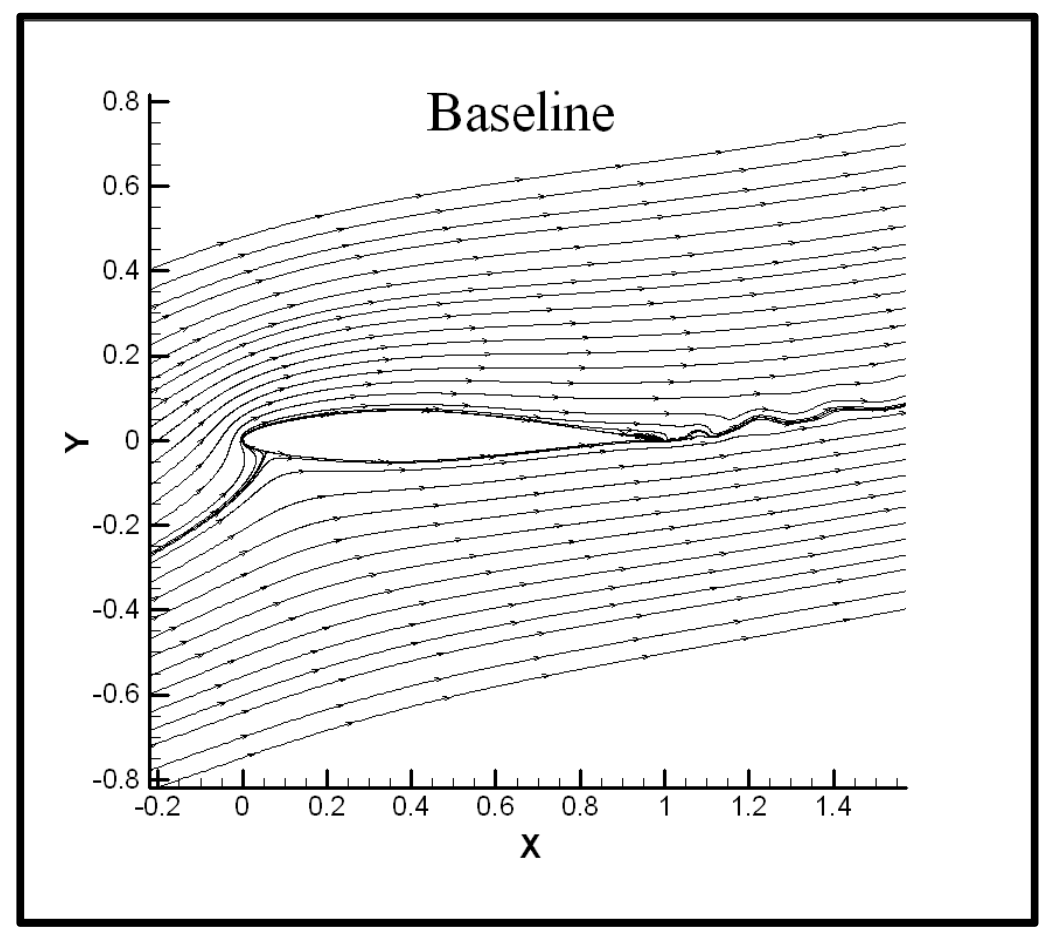

Figure 33. Baseline airfoil at 15 degrees with separation at $0.86 \mathrm{c}$

The boundary layer profiles at $0.95 \mathrm{c}$ for baseline and models (b) and (ab) are plotted in Figures 34 and 35. The model (ab) with the most improvement has no reversed flow or circulation at the trailing-edge when compared with the baseline airfoil. A close-up view of the boundary layer is given in Figure 35.

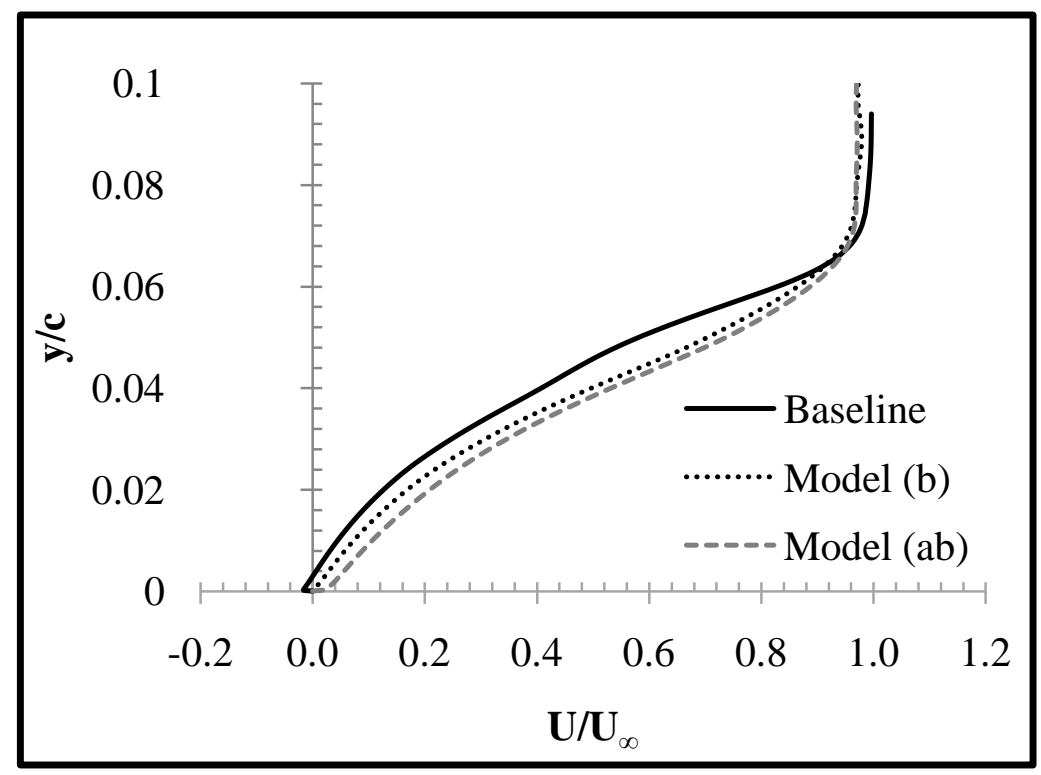

Figure 34 . Boundary layer comparison at $0.95 \mathrm{c}$ at $\mathrm{AOA}$ of $15^{\circ}$ 


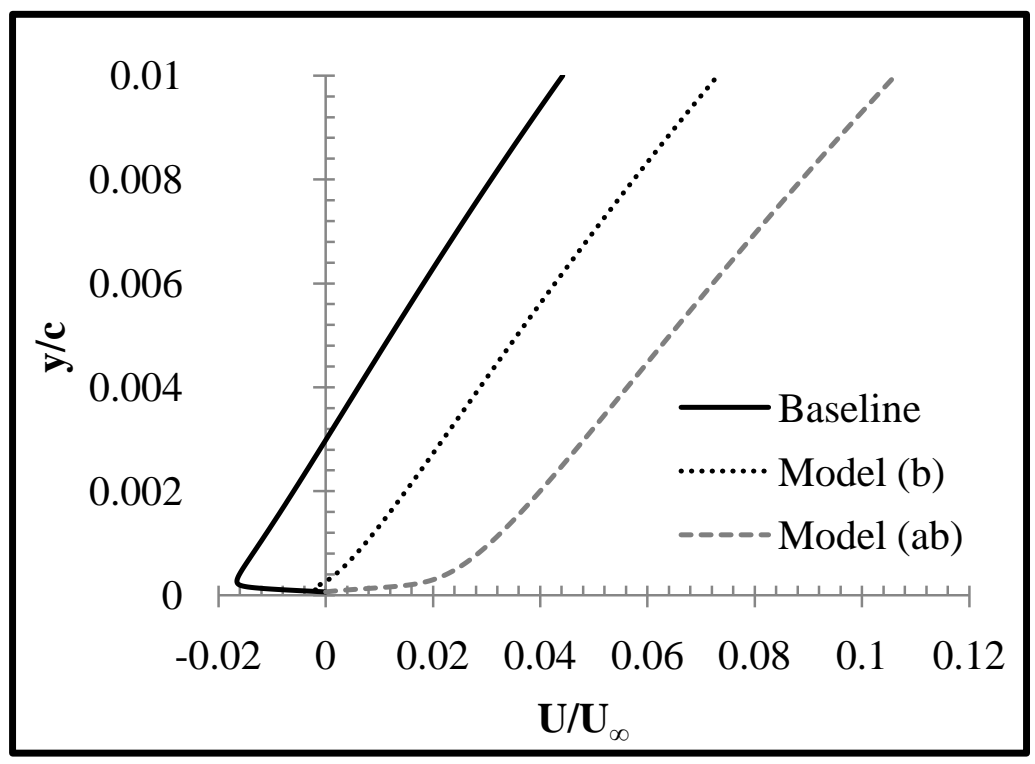

Figure 35 . Boundary layer comparison at $0.95 \mathrm{c}$ close to surface at $\mathrm{AOA}$ of $15^{\circ}$

Effects of the best Stall vane model on static and dynamic pressure are shown in Figures 36 and 37 compared with the baseline. Note that pressure fluctuations induced by flow separation, on the baseline airfoil, are significantly reduced downstream on the model (ab). However, the Stall vane has an impact on the main airfoil pressure distribution. This causes the $C_{l \max }$ on the model (ab) to decrease by $1.0 \%$, while the measured $C_{l}$ time history fluctuates around $\pm 0.25 \%$. The baseline airfoil, $C_{l \max }$ is 1.659 , as for the model (ab) is 1.641 at 1.0 second of run-time (i.e., $1.0 \%$ ).

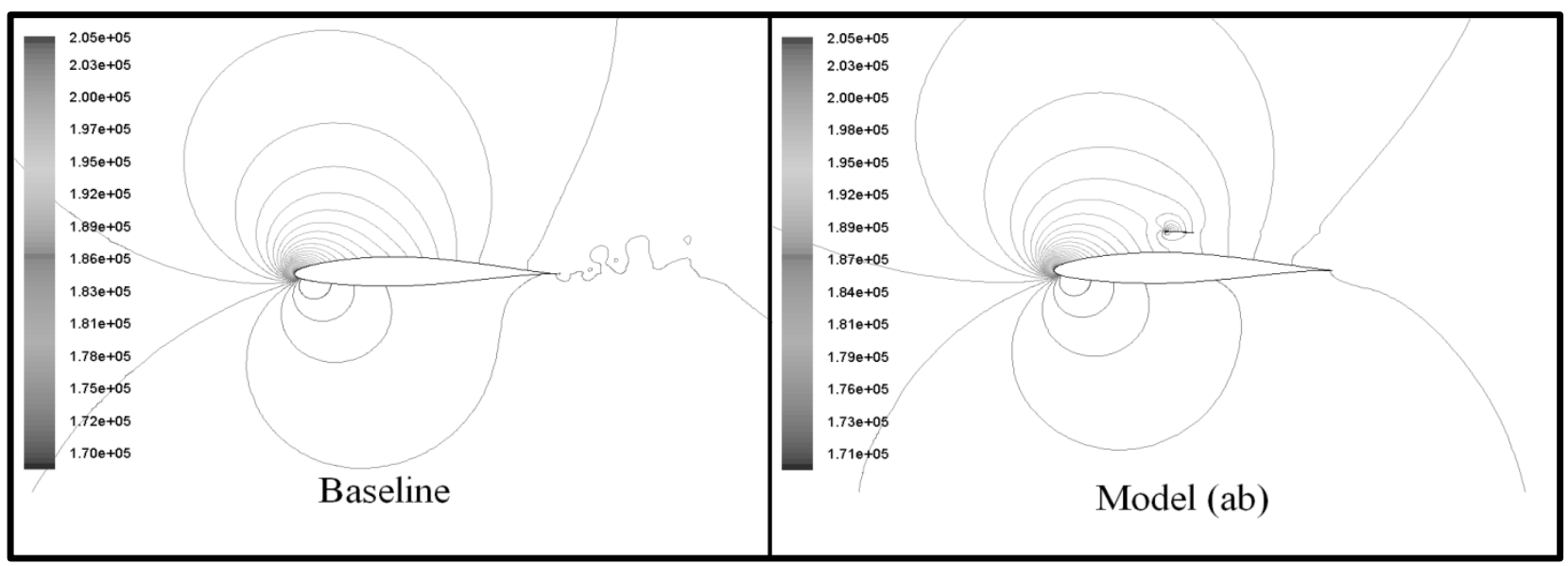

Figure 36. Static pressure contour at AOA of $15^{\circ}$ (in Pascal) 
Perturbations can be identified in dynamic pressure contours (Figure 37) over the baseline airfoil. In contrast, a uniform flow at the trailing-edge of model (ab) verifies the previous results.

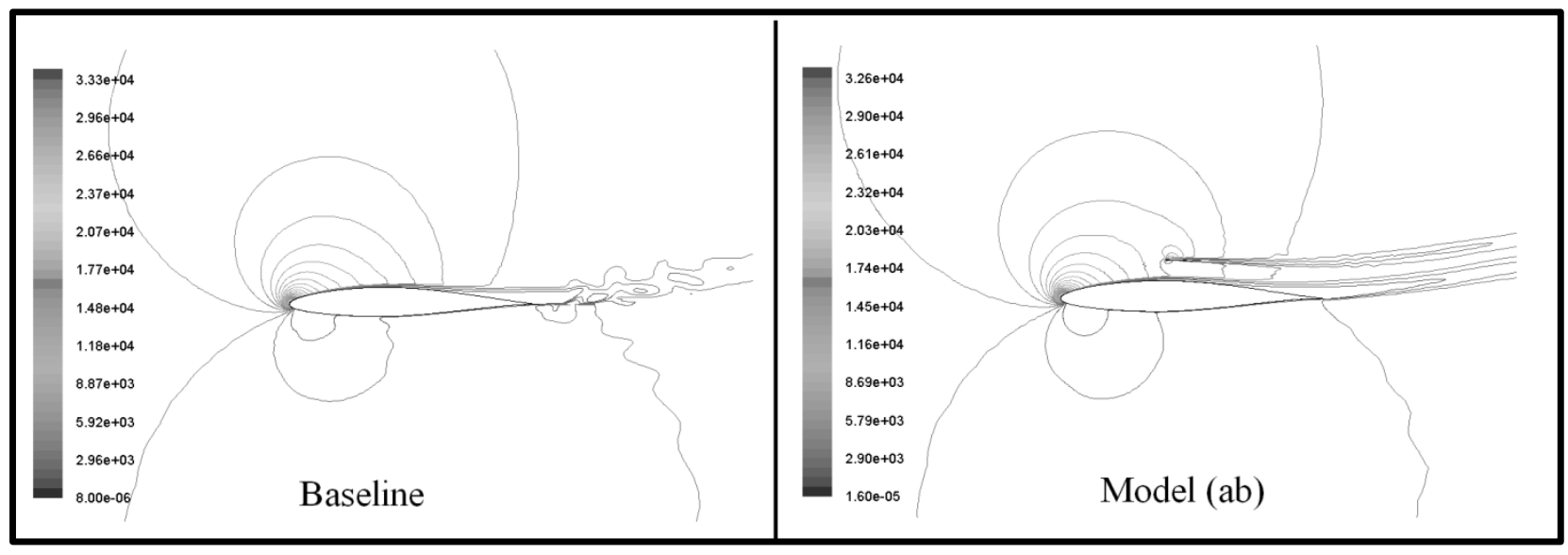

Figure 37. Dynamic pressure contour at AOA of $15^{\circ}$ (in Pascal)

Maximum turbulence intensity, as shown in Figure 38, has increased on model (ab) due to the flat, sharp leading-edge of the device. At $15^{\circ}$ angle-of-attack, the baseline airfoil has an instantaneous drag coefficient of 0.0329 while the model (ab) main element is 0.0142 , but the total drag coefficient of 0.0424 including the Stall vane. This result is due to the vane's sharp leadingedge and separated flow, which dominates the entire upper surface.

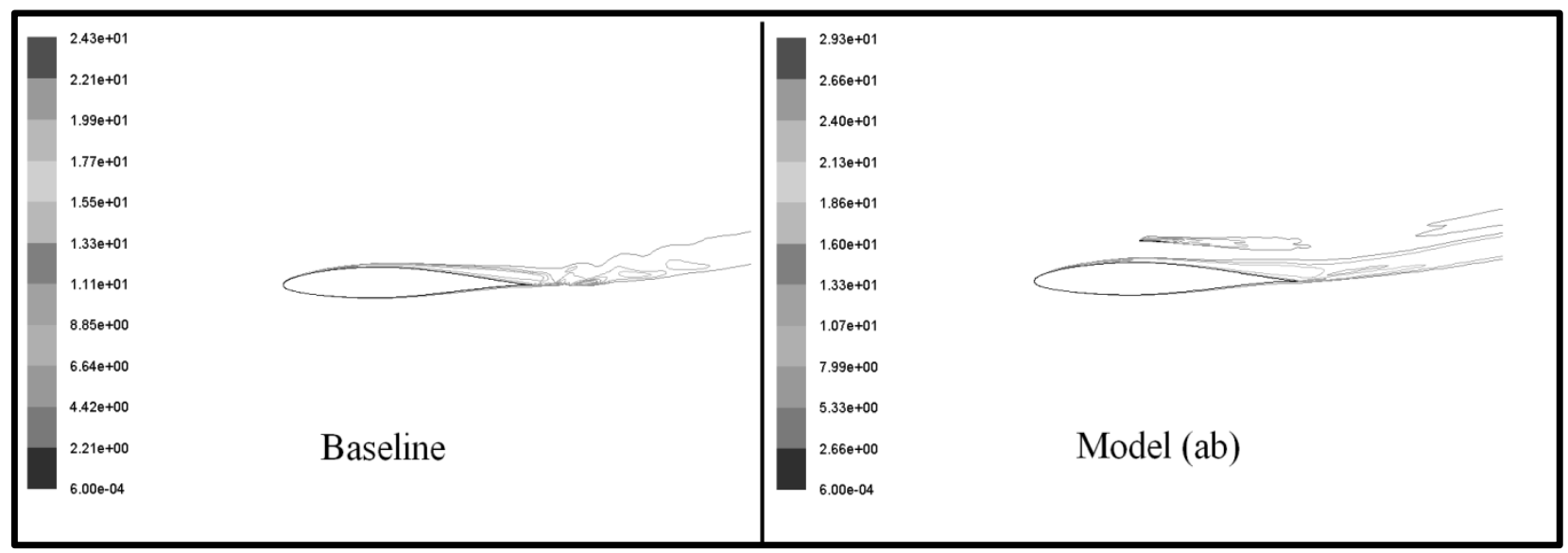

Figure 38 . Turbulence intensity contour at AOA of $15^{\circ}$ (in \%) 
The total turbulent viscosity ratio $\mu_{\mathrm{t}} / \mu$ is decreased on model (ab) by delaying the separation on the main surface. Figure 39 shows dense turbulent viscosity at the baseline trailing-edge where the flow separation has taken place.

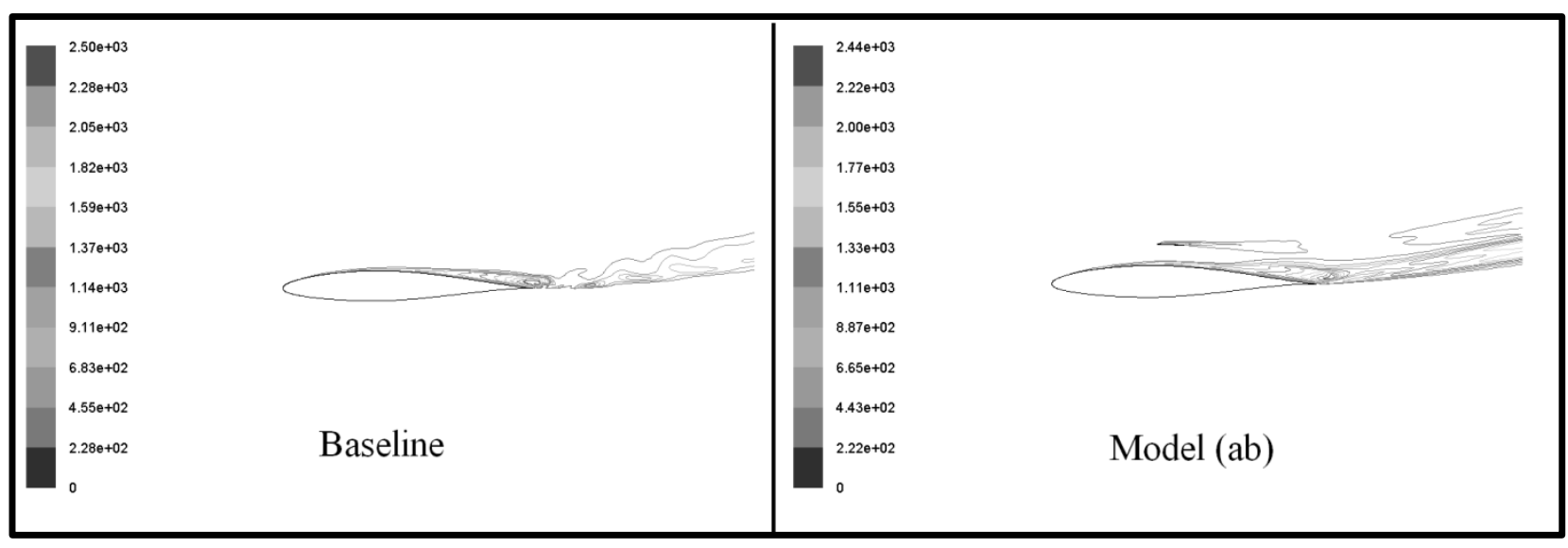

Figure 39. Turbulent viscosity ratio $\mu_{\mathrm{t}} / \mu$ contour at AOA of $15^{\circ}$ 


\subsection{Cylinder}

The device analysis matrix, for the cylinder concept, is provided in table 5. In contrast to the previous section, likely dependents for this configuration are not as easy to identify. $\underline{\text { Hence, a trial- }}$ and-error method is utilized to show the model total $C_{l}, C_{d}$ and $C_{m}$ dependency, as well as the flow separation point. Figure 40 shows the model with corresponding $X, Y$ and $R$ arrangements, where $(y)$ is the gap between the cylinder and the airfoil.

TABLE 5

ANALYSIS MATRIX FOR CYLINDER MODEL

\begin{tabular}{ccccc}
\hline \hline model & $\boldsymbol{X}$ & $\boldsymbol{R}$ & $\boldsymbol{Y}$ & $(\boldsymbol{y})$ \\
\hline $\mathbf{C 1}$ & 0.4 & 0.01 & 0.1 & $(0.02)$ \\
$\mathbf{C 2}$ & 0.4 & 0.005 & 0.095 & $(0.02)$ \\
$\mathbf{C 3}$ & 0.4 & 0.005 & 0.085 & $(0.0095)$ \\
C2_2c & 0.2 & 0.005 & 0.095 & $(0.02)$ \\
C2_3c & 0.3 & 0.005 & 0.095 & $(0.02)$ \\
C2_5c & 0.5 & 0.005 & 0.095 & $(0.02)$ \\
C2_6c & 0.6 & 0.005 & 0.095 & $(0.02)$ \\
\hline \hline
\end{tabular}

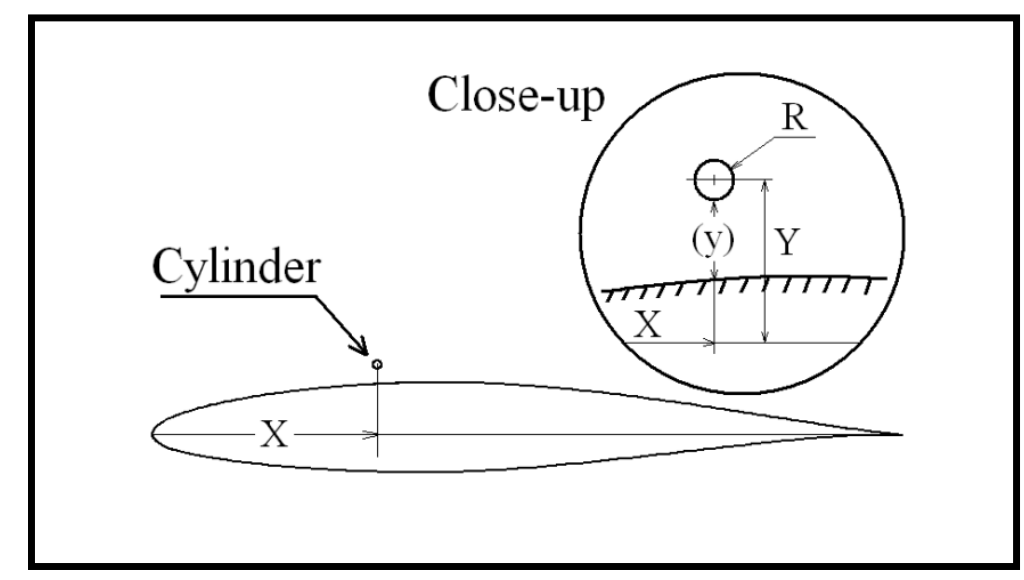

Figure 40. Cylinder design model 
The cylinder's diameter is tested for the first two models: C1 and C2. Based on the results, the superior model is taken to the next assessment. Note that the cylinder radius has two different values of $0.005 \mathrm{c}$ and $0.01 \mathrm{c}$.

A total of seven models are simulated at three different angles-of-attack. Section lift coefficient, $C_{l}$ against angle-of-attack is presented in Figure 41. Drag and pitching moment coefficients against $C_{l}$ are shown in Figure 42. As the cylinder moves toward the trailing-edge, the lift curve improves, but it is still inferior to the baseline. On the other hand, a larger cylinder diameter and a smaller gap (y) have negative effects on the drag and moment coefficients.

Further investigations of streamline contours, as shown in Figures 43-45, show the trailingedge flow separation at $15^{\circ}$ of angle-of-attack. No flow separation delay is detected on any cylinder model. In fact, cylinder models have unconstructive influence that induces flow separation earlier and causes an increased drag, when compared with the baseline airfoil.

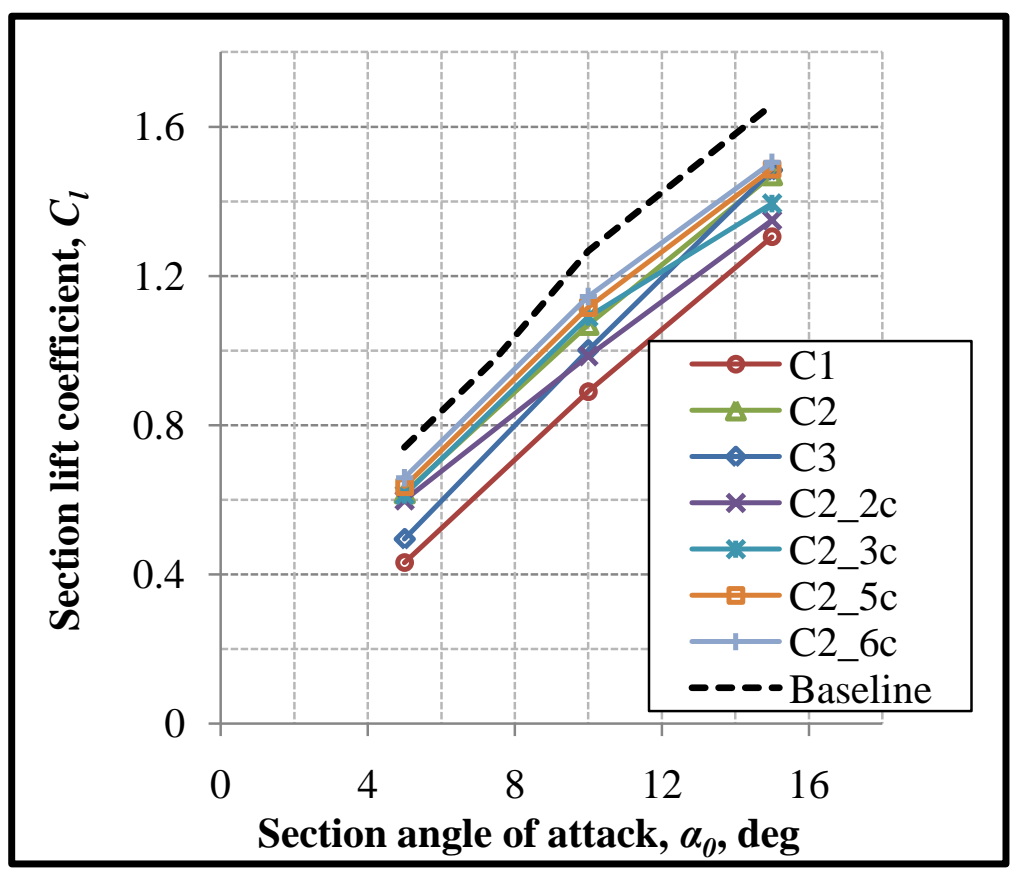

Figure 41. Cylinder section lift coefficient at $\mathrm{Re}=6.0 \times 10^{6}$ 


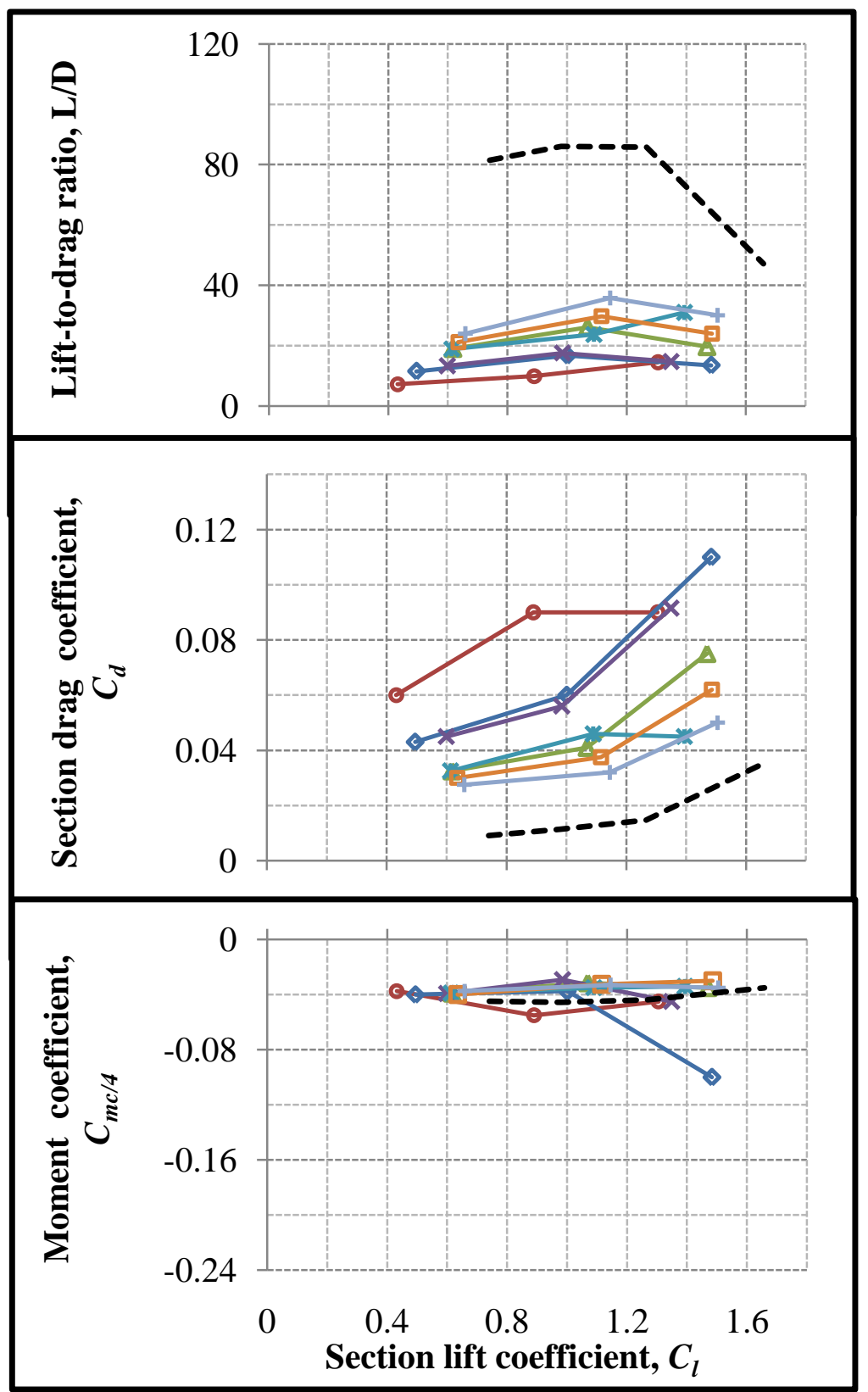

Figure 42. Cylinder lift-to-drag ratio, drag polar and quarter chord moment coefficient at $\operatorname{Re}=6.0 \times 10^{6}$ 


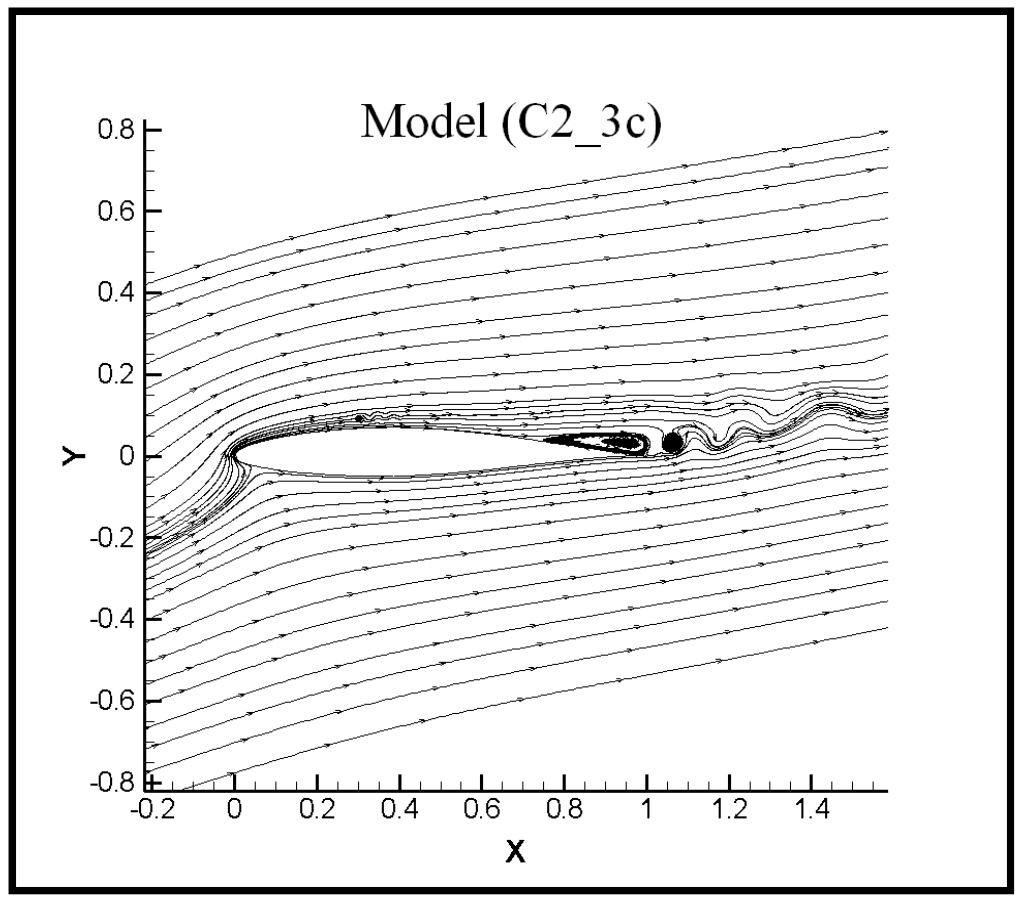

Figure 44. Model (C2_3c) at 15 degrees with separation at $0.70 \mathrm{c}$

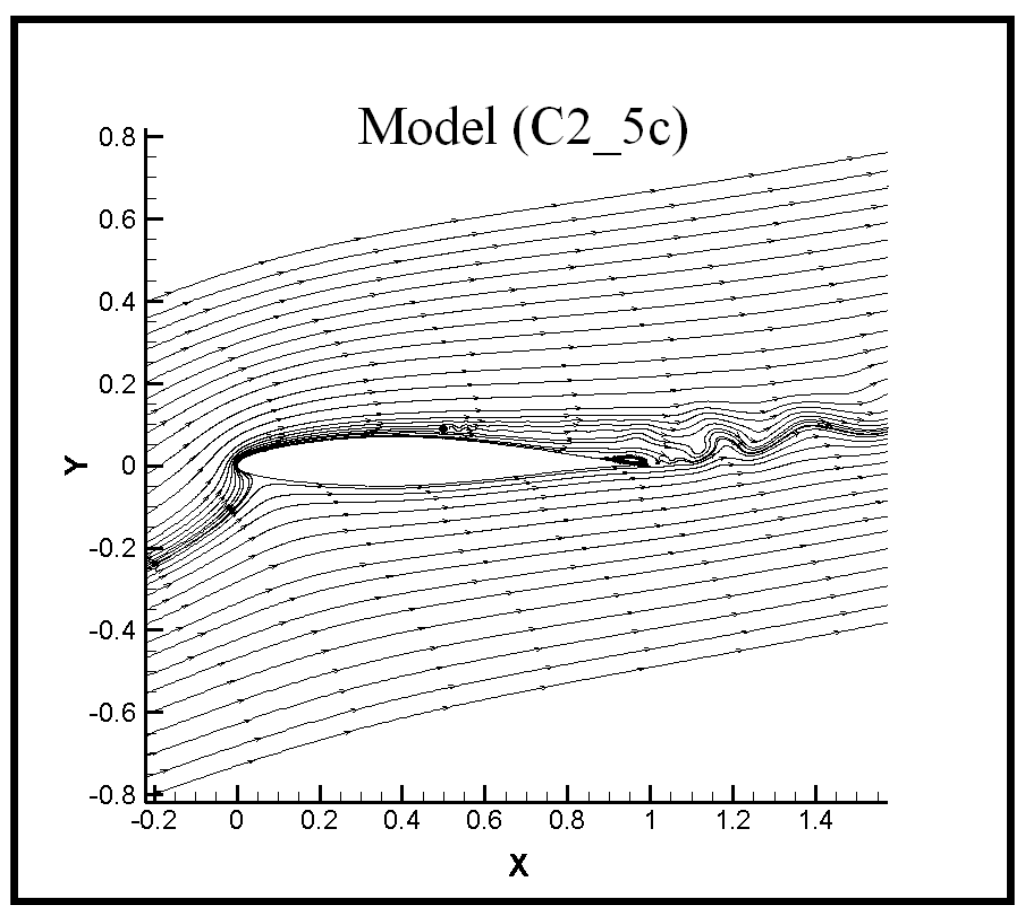

Figure 43. Model (C2_5c) at 15 degrees with separation at $0.82 \mathrm{c}$ 


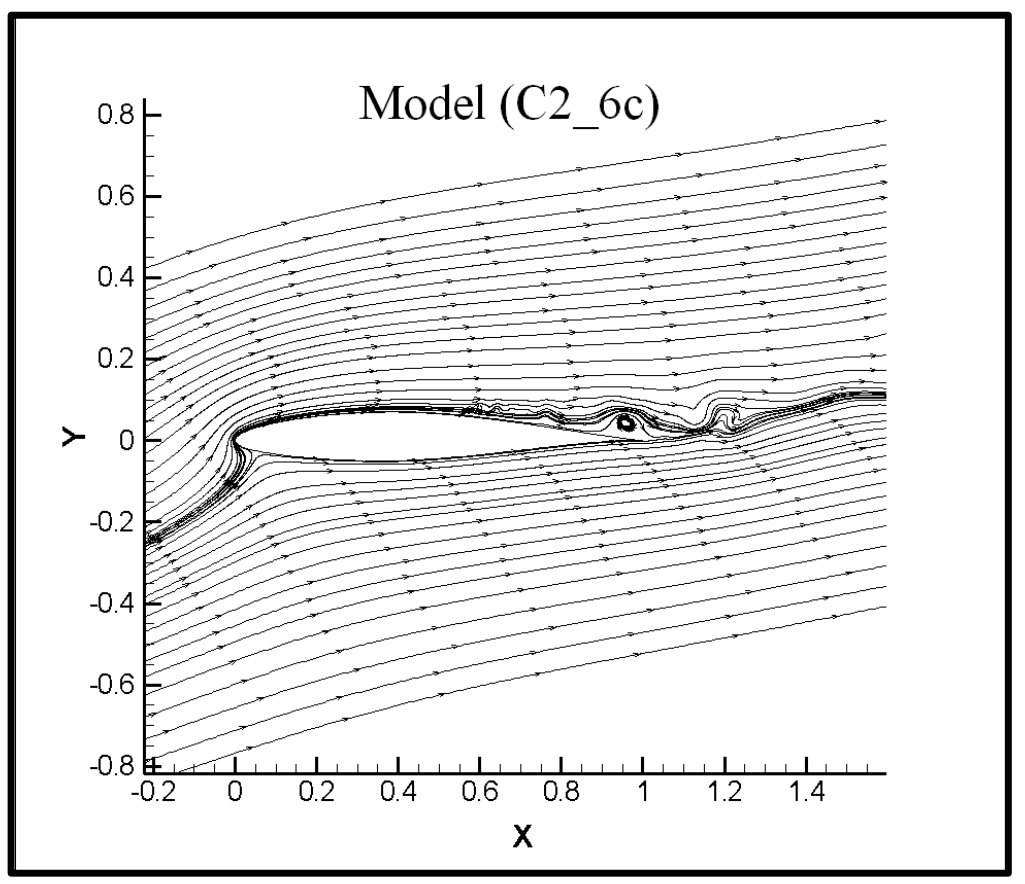

Figure 45. Model (C2_6c) at 15 degrees with separation at 0.9c

Although the separation on the surface of model (C2_6c) seems to be delayed in Figure 45, the off-surface vortex is causing the establishment of a retarded boundary layer. Boundary layer profiles at $0.95 \mathrm{c}$ for baseline and models $\left(\mathrm{C} 2 \_3 \mathrm{c}\right),\left(\mathrm{C} 2 \_5 \mathrm{c}\right)$ and $\left(\mathrm{C} 2 \_6 \mathrm{c}\right)$ are plotted in Figures 46. The model (C2_6c) has the largest reversed flow region close to the surface. However, the model recovers the boundary layer earlier than other two models.

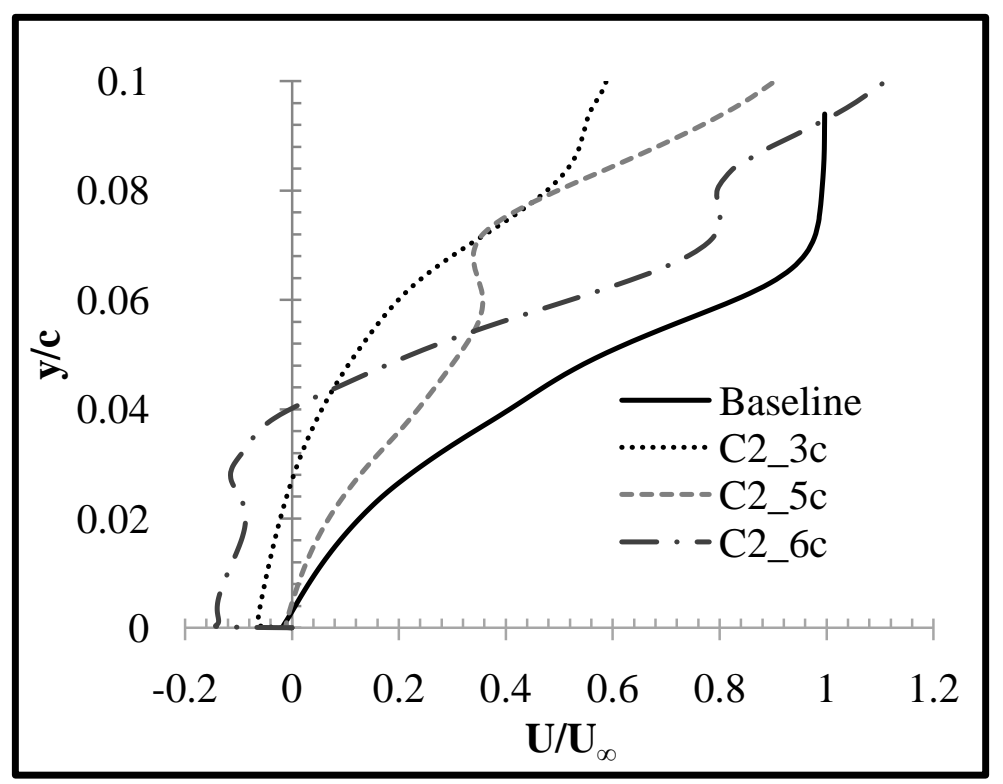

Figure 46 . Boundary layer comparison at $0.95 \mathrm{c}$ at $\mathrm{AOA}$ of $15^{\circ}$ 
The cylinder wake is not only causing an increase in drag but also initiating flow separation as stated above. This fact can be observed in pressure contours in Figure 47 as well. In contrast to the Stall vane model, pressure is rapidly changing close to the trailing-edge generating a strong shear layer at this region (see Figure 47 right).

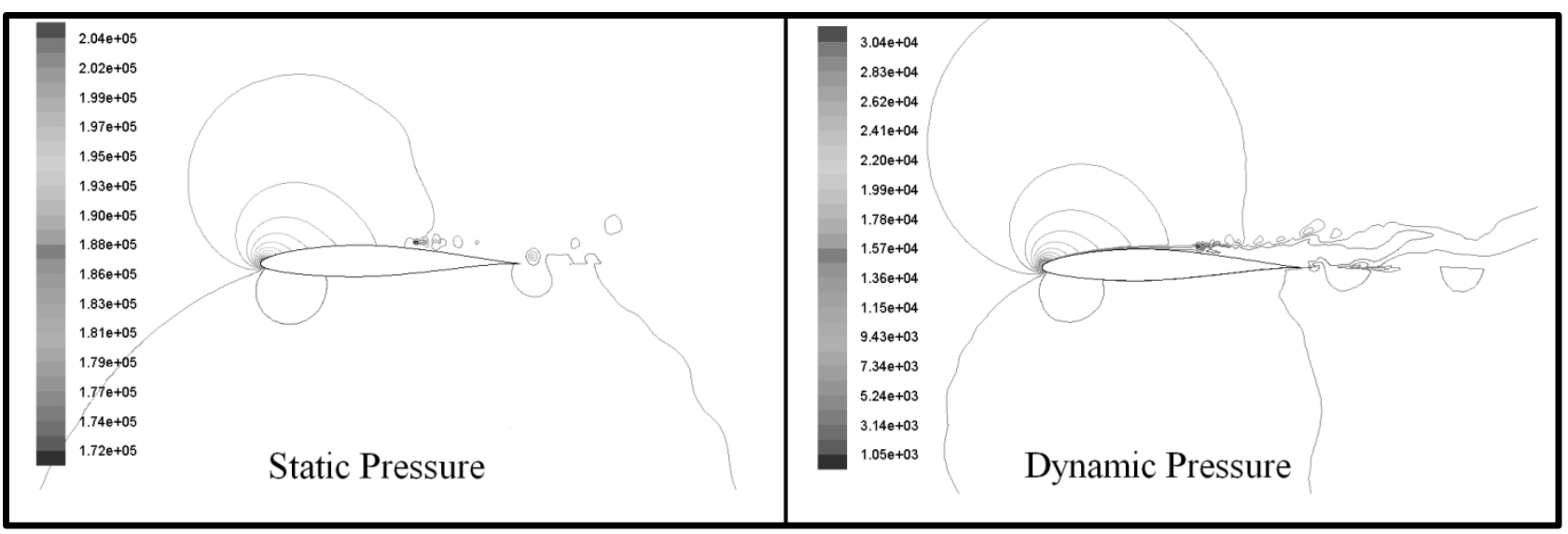

Figure 48. Pressure contours over model (C2_6c) at AOA of $15^{\circ}$ (in Pascal)

As shown in Figure 48, despite a large increase in drag, the turbulent viscosity ratio and turbulence intensity drop significantly when compared with the baseline (refer to the Figures 38 , 39).

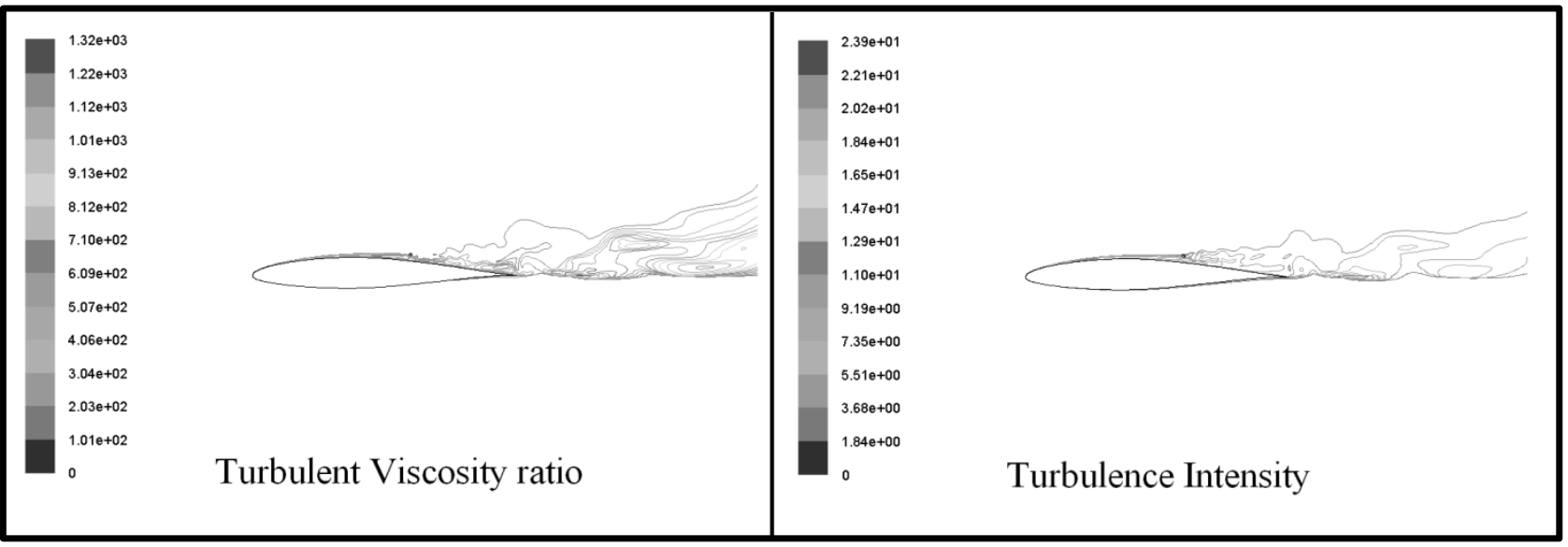

Figure 47. Model (C2_6c) turbulent viscosity ratio and turbulence intensity (\%) contours at AOA of $15^{\circ}$ 


\subsection{Dimple}

The device analysis matrix for the Dimple configuration is presented in table 6. Two factors are considered to be the most influential: $x$-location and $d$, as the diameter of the dimple. In addition, two models of four dimples at different diameters are evaluated. Dimples are located before and after the maximum thickness of the airfoil so that laminar flow close to the leading-edge is preserved. Two levels (+/-) for each factor show the model dependency on $C_{l}, C_{d}$ and $C_{m}$, as well as the flow separation point. Figure 49 shows the model identifying the corresponding $x$ and $d$ arrangements.

TABLE 6

ANANLYSIS MATRIX FOR DIMPLE MODEL

\begin{tabular}{ccc||ccc}
\hline \hline model & $\mathbf{x}$ & $\mathbf{d}$ & & - & + \\
\hline $\mathbf{1}$ & - & - & $\mathbf{x}$ & $0.3 \mathrm{c}$ & $0.75 \mathrm{c}$ \\
$\mathbf{a}$ & + & - & $\mathbf{d}$ & $0.05 \mathrm{c}$ & $0.1 \mathrm{c}$ \\
$\mathbf{b}$ & - & + & & & \\
$\mathbf{a b}$ & + & + & & & \\
4_5d & - & - & & & \\
4_10d & - & + & & & \\
\hline \hline
\end{tabular}

Note: $\mathrm{dx}=0.15 \mathrm{c}, \mathrm{R}=\mathrm{d}, \mathrm{r}=\mathrm{d} / 10$.

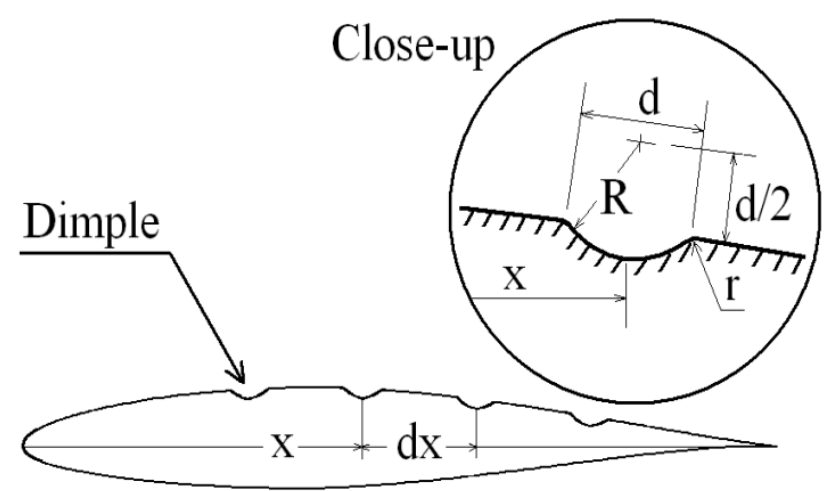

Figure 49. Dimple design model 
A total of six models are simulated at four angles-of-attack. Section lift coefficient, $C_{l}$ against angle-of-attack is presented in Figure 50. Lift-to-drag ratio, drag and pitching moment coefficients against $C_{l}$ are shown in Figure 51. Clearly, as the dimples move toward the leadingedge, the maximum lift decreases, and the drag increases. Meanwhile, dimples closer to the airfoil trailing-edge have less significance on the total lift and drag when compared to the baseline. Nevertheless, a slight improvement in the model (a) lift-to-drag ratio is detected, but this is near to the range of measured $C_{l}$ time history fluctuation. Further investigations need to be implemented to validate these results.

Streamline contours, Figures 52-53, show trailing-edge flow separation at $15^{\circ}$ of angle-ofattack. No flow separation delay is detected on the dimple models. Dimples with $10 \%$ chord length diameter initiate flow separation instantly, with an increase in drag. Not one of the four-dimplemodels has a favorable effect on delaying the separation.

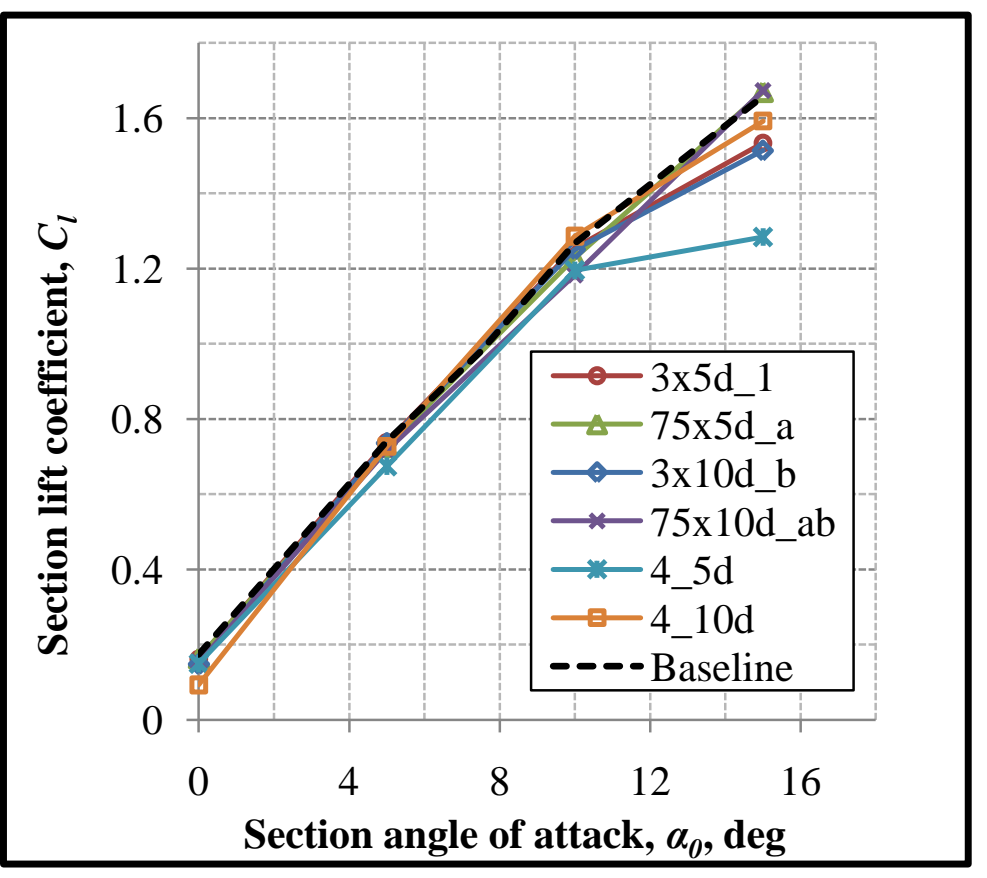

Figure 50. Dimples section lift coefficients at $\mathrm{Re}=6.0 \times 10^{6}$ 


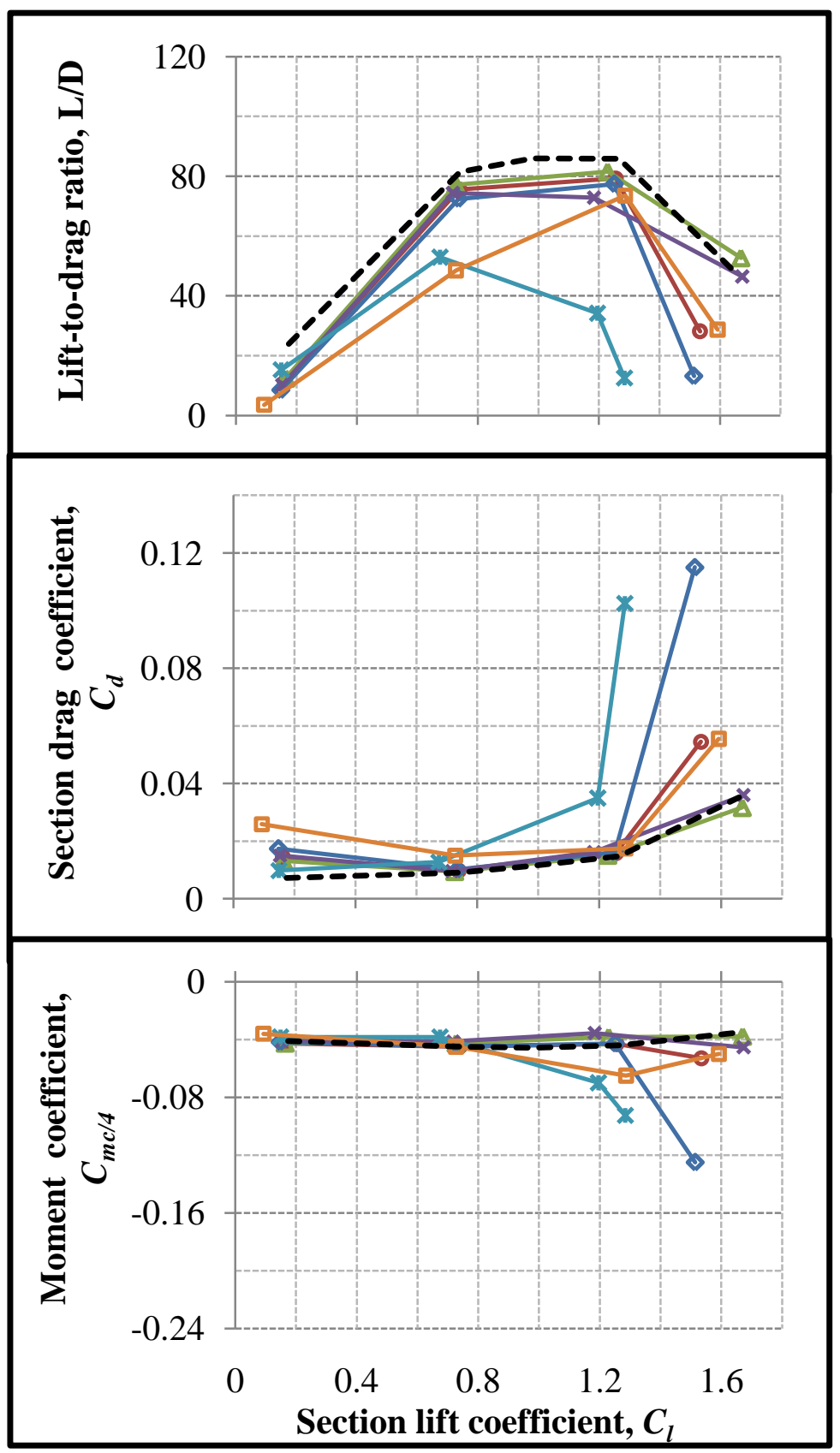

Figure 51. Dimples lift-to-drag ratio, drag polar and quarter chord moment coefficient at $\operatorname{Re}=6.0 \times 10^{6}$ 


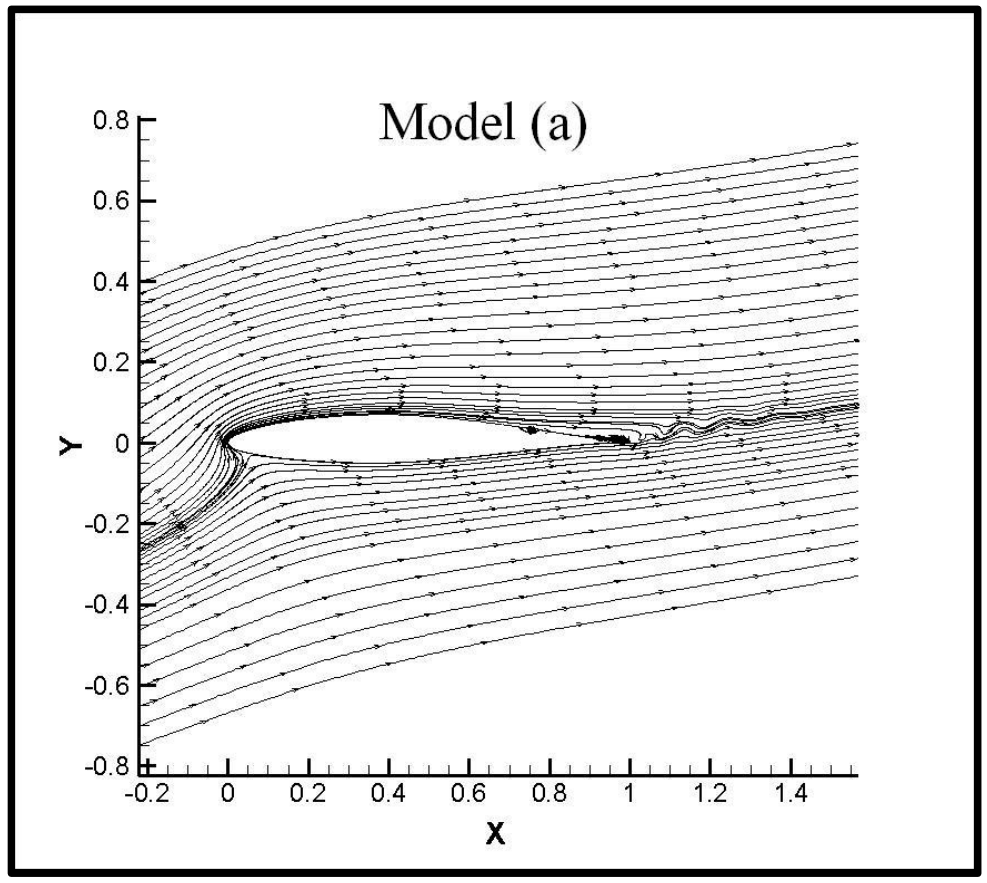

Figure 53. Model (a) at 15 degrees with separation at $0.84 \mathrm{c}$

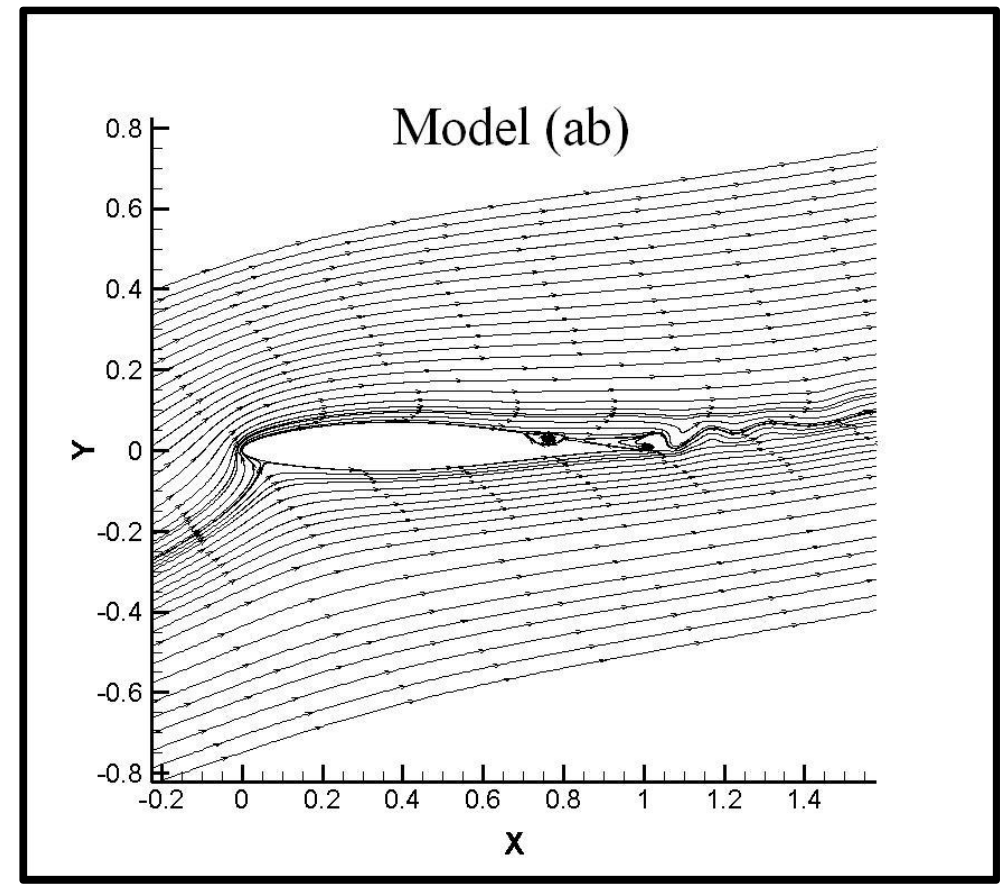

Figure 52. Model (ab) at 15 degrees with separation at $0.80 \mathrm{c}$ 
The boundary layers of the two-dimple-models are compared with the baseline in Figure 54. Model (ab) shows larger separation in the $y$-direction, but maximum velocity is augmented at the edge of the boundary layer. Model (a) does not show any significant change compare to the baseline.

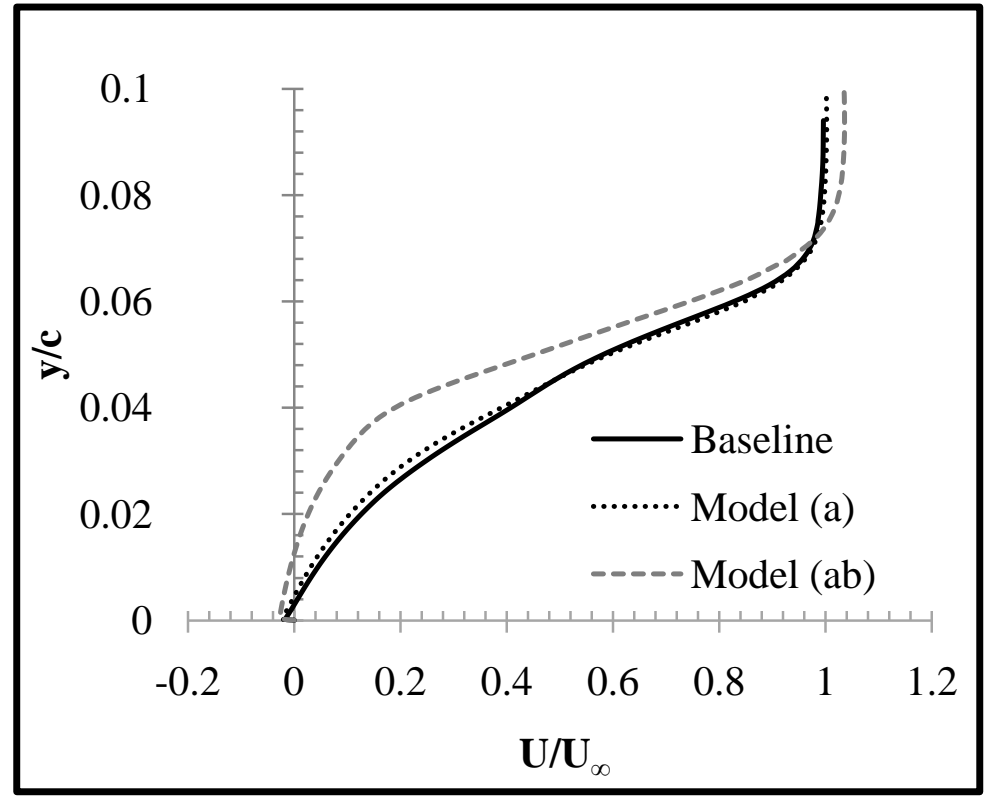

Figure 54 . Boundary layer comparison at $0.95 \mathrm{c}$ at $\mathrm{AOA}$ of $15^{\circ}$

The static and dynamic pressure contours of the models (a), (ab) and the baseline airfoil are plotted in Figures 55-57. There is no clear result indicating that dimple models improve the stall characteristics. Yet, models (a) and (ab) at higher angles-of-attack (Figure 51) show improvements in lift-to-drag ratio. An overall reduction of dynamic pressure, compared to the two tested models, verifies a loss of energy in the boundary layer. 


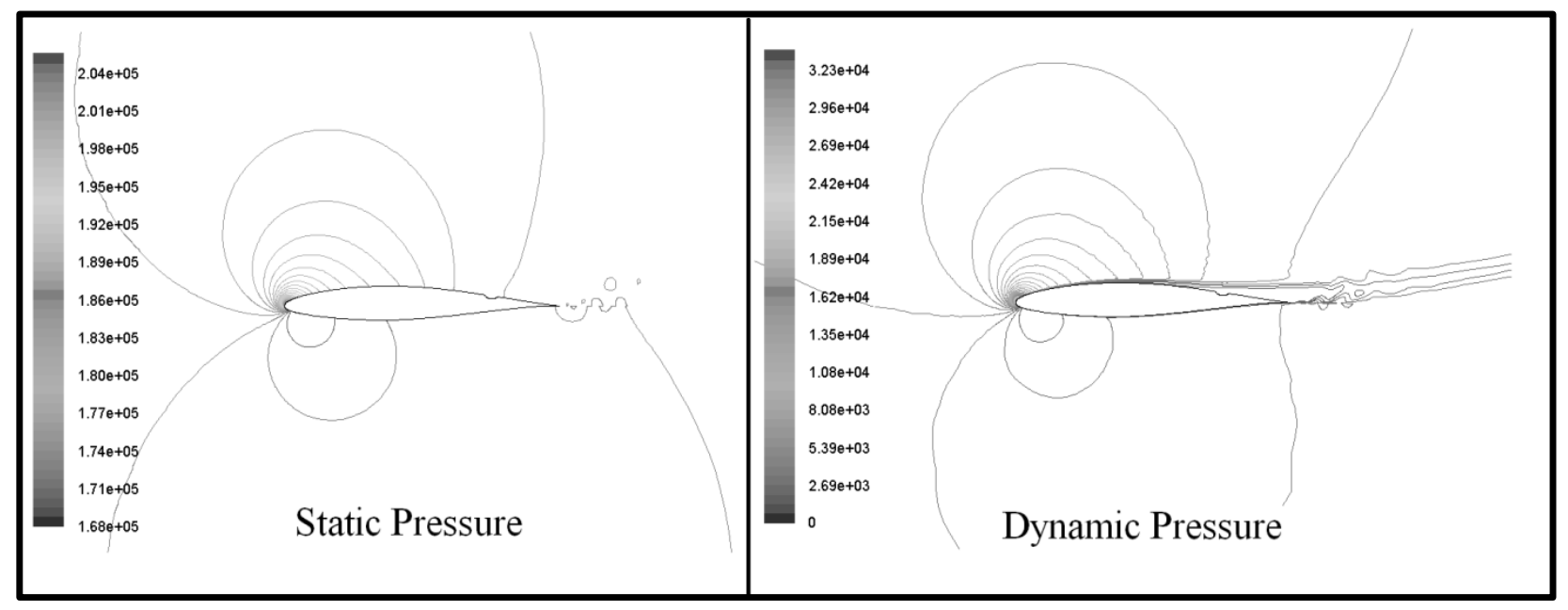

Figure 57. Model (a) pressure contours (in Pascal) at AOA of $15^{\circ}$

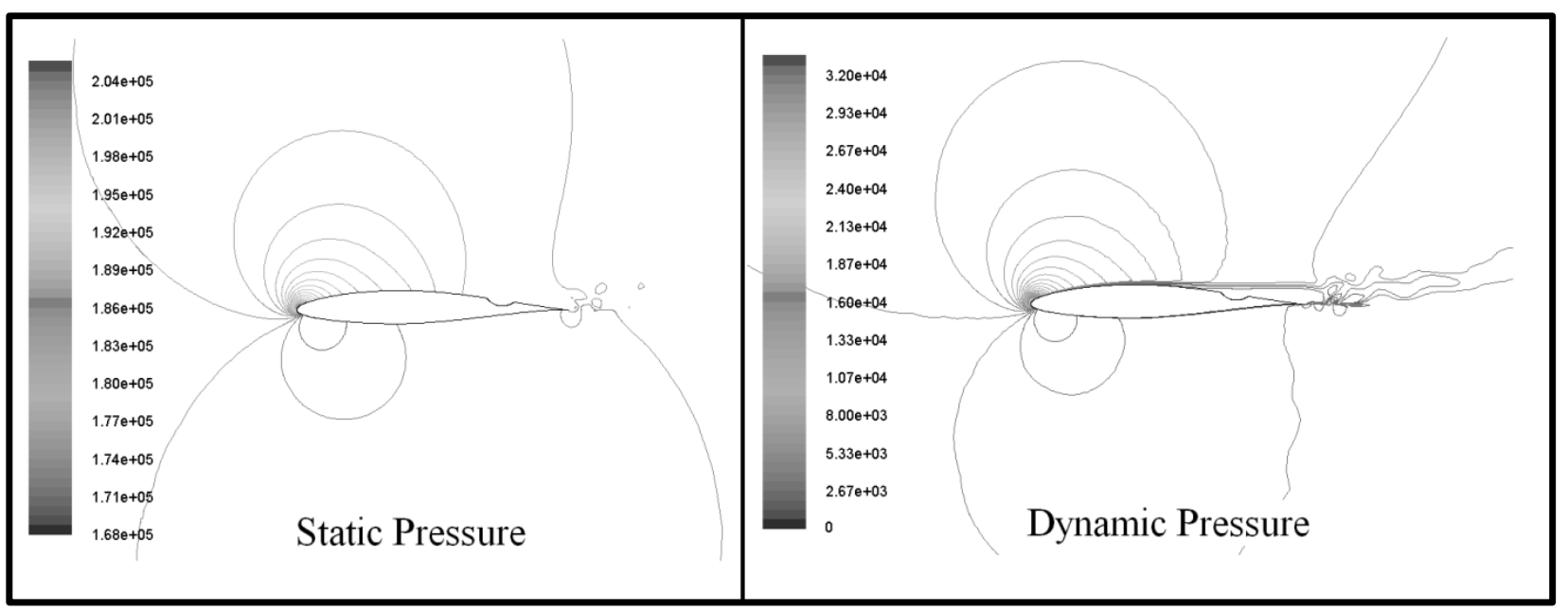

Figure 56. Model (ab) pressure contours (in Pascal) at AOA of $15^{\circ}$

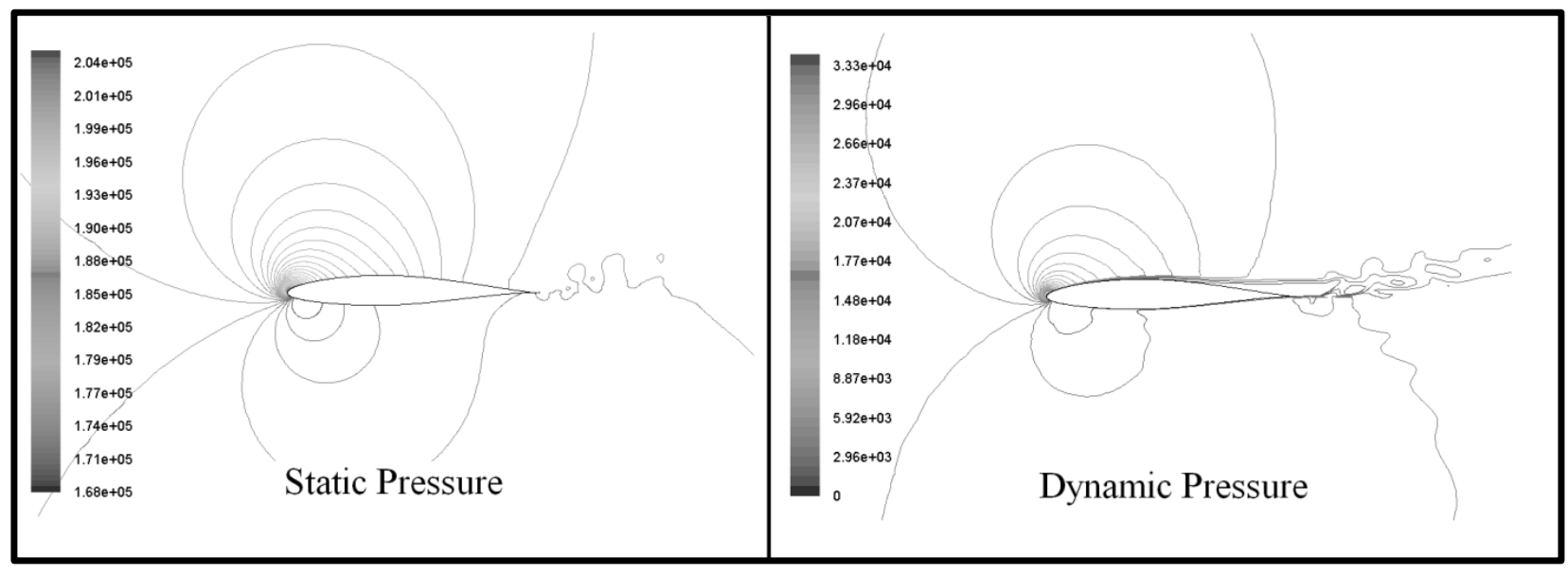

Figure 55. Baseline airfoil pressure contours (in Pascal) at AOA of $15^{\circ}$ 
The turbulence intensity of model (a) and (ab) are presented in Figure 58. A small increase in maximum turbulence intensity on both models (a), with $24.5 \%$, and (ab) with $24.4 \%$, is detected. However, they can be considered insignificant since they represent a less than $0.2 \%$ increase from the baseline. Turbulent viscosity ratio is dramatically decreased on model (ab) with $1.44 \times 10^{3}$, approximately half of the value on the baseline airfoil. This can also be said for the cylinder model (C2_6c). Figure 59 shows the turbulent viscosity ratio on models (a) and (ab).

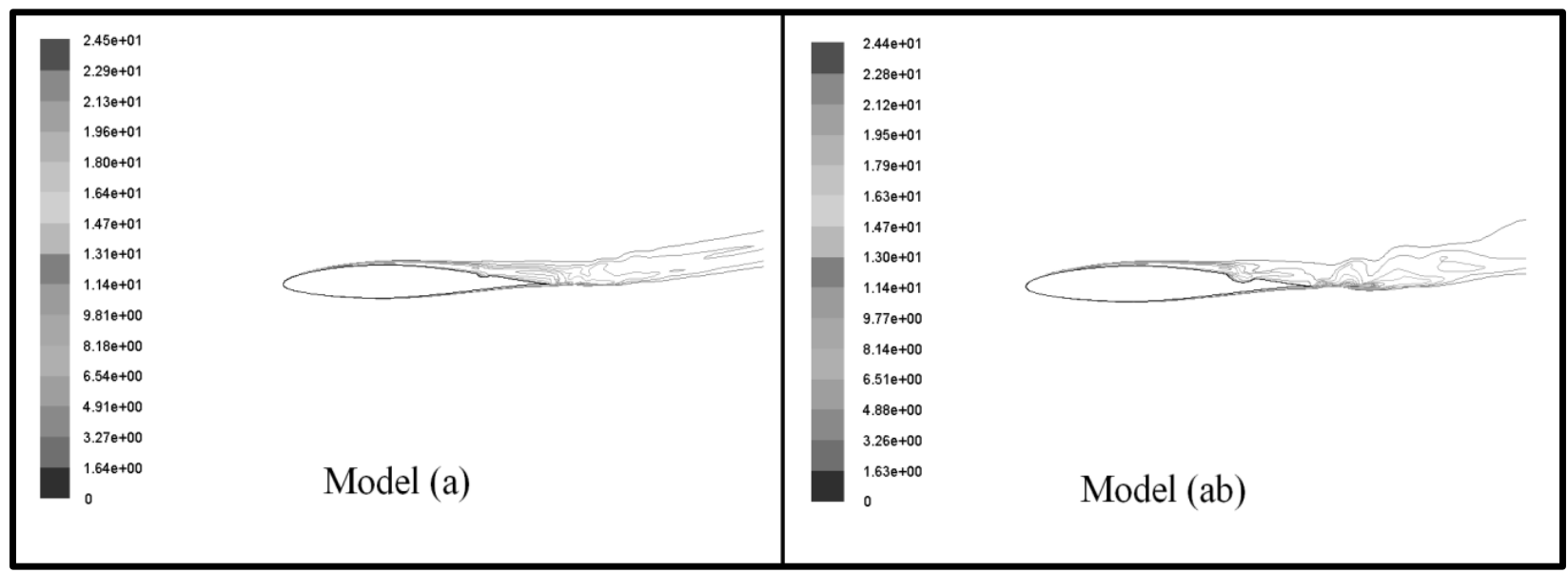

Figure 58. Turbulence intensity contours (in \%) at AOA of $15^{\circ}$

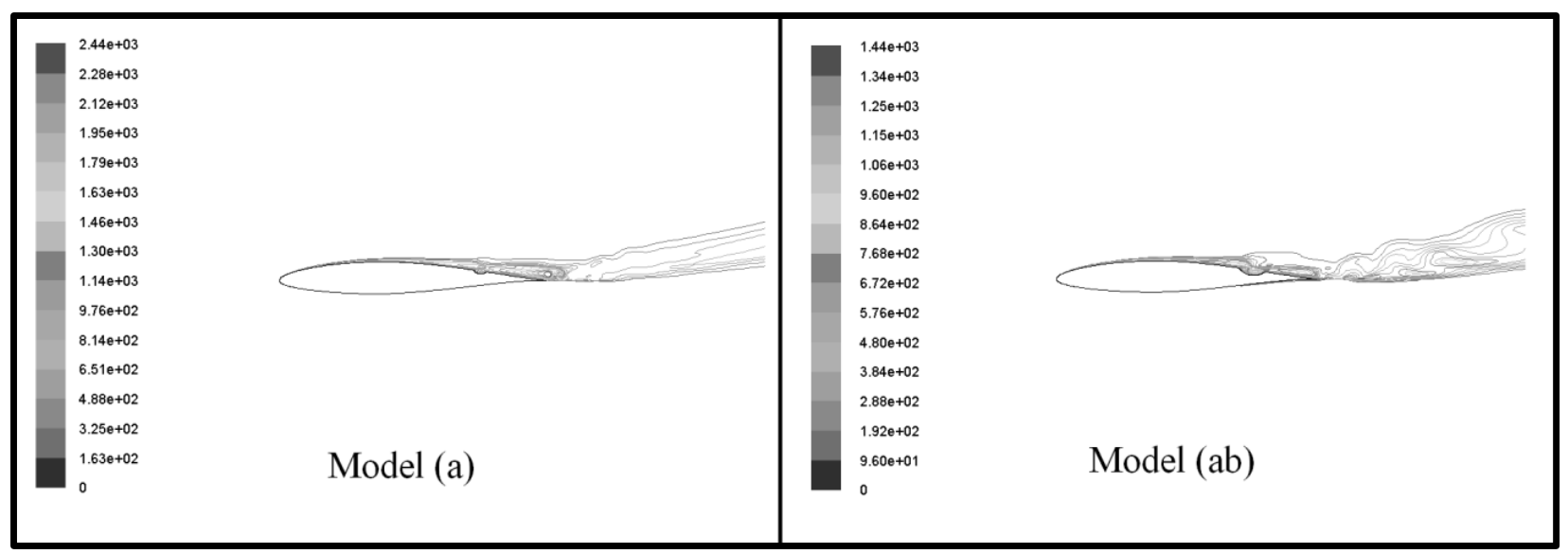

Figure 59. Turbulent viscosity ratio $\mu_{\mathrm{t}} / \mu$ contour at AOA of $15^{\circ}$ 


\section{CHAPTER 6}

\section{CONCLUSIONS}

Three simple devices were tested, using a DDES turbulence model, to evaluate their effect on separation delay and increases in lift-to-drag ratio. Impact on drag and pitching moment are also

presented at the flight condition of $\operatorname{Re}=6.0 \times 10^{6}$. Results for each conceptual design were compared against a single-element airfoil.

The cylinder model had the poorest results in terms of separation delay, lift-to-drag ratio and $C_{l}$ against $\alpha$. The boundary layer plots and pressure contours justify the conclusion that a cylinder wake initiates early flow separation. The dimple model (a) is the best among other dimple configurations, but it shows no significant improvement when compared to the baseline. However, its lift-to-drag ratio improves at a higher angle-of-attack. The model (ab) turbulent viscosity ratio decreases by $42 \%$ from the baseline. This shows that small-scale eddies have less effect on the viscosity dissipation; instead, large eddy viscosity is at its highest on the trailing-edge's free shear layer. This result indicates less skin friction drag but higher pressure drag for this model.

The overall best model was the Stall vane model (ab), where no flow reversal, or separation, is detected on the main airfoil. However, lift-to-drag ratio did not improve due to the sharp leadingedge separation on the device. The flat plate shape of the stall vane causes a large portion of the total drag (66\%). Although the main airfoil drag decreases by $43 \%$, the total drag, including the device, increases by $22 \%$ from the baseline. No significant $C_{l \max }$ change is detected. 


\section{CHAPTER 7 \\ RECOMMENDATIONS}

Three conceptual designs were tested. Suggestions for future optimizations and testing are as follows:

- Turbulence model validation

The DDES turbulence model needs to be validated using a 3D grid domain for a slatted airfoil at higher angles-of-attack. Evidently, the DDES is the best available turbulence model, but pursuit of accuracy has to be followed by a more realistic meshing environment where the zdirection is also included. Next, using a dynamic or moving mesh may more accurately visualize stall, since it is a continuous process. In the CFD, each simulation represents one boundary condition (i.e., angle-of-attack). A moving mesh can allow for a continuously changing angle-ofattack. Consequently, the device's effect on separation delay can be obtained more accurately.

\section{- Model optimizations}

Instead of using a flat plate for the stall vane model (ab), a symmetric airfoil shape device should be used to reduce the drag. Meanwhile, the device thickness is suggested to be minimized for less effect on the pressure distribution of the main airfoil. It is also recommended to evaluate the device characteristics on a dynamic stall, by using the moving mesh technique.

The dimple model (a), positioned in the pressure recovery region, seems to be fairly effective. Further investigation is suggested at higher angles-of-attack and possibly with an increase in dimple numbers.

- Experimental evaluations

A series of experiments should be performed to further study and evaluate this investigation's concepts and results. 
REFERENCES 


\section{REFERENCES}

[1] M. Gad-el-Hak, Flow Control Passive: Active and Reactive Flow Management, First ed., Cambridge University Press, 2000.

[2] D. J. Butter, "Recent Progress on Development and Understanding of High Lift Systems," in Improvement of Aerodynamic Performance Through Boundary Layer Control and High Lift Systems, Brussels, Belgium, pp. 1.1-1.26, 1984.

[3] J. D. Anderson, Fundamentals of Aerodynamics, McGraw Hill Series, Third Edition, pp. 4147, 2001.

[4] A. M. O. Smith, "High-Lift Aerodynamics," Journal of Aircraft vol. 12, pp. 501-530, 1975.

[5] R. H. Liebeck, Smyth, D.N., "Study of Slat-Airfoil Combinations Using Computer Graphics," Journal of Aircraft, vol. 10, April, pp. 254-256, 1973.

[6] M. Sajben, et al., "A New, Passive Boundary Layer Control Device," AIAA-1976-700, presented at the American Institute of Aeronautics and Astronautics and Society of Automotive Engineers, Propulsion Conference, 12th, Palo Alto, Calif, 26-29 July, 1976.

[7] F. G. Howard and W. L. Goodman, "Drag Reduction on a Bluff Body at Yaw Angles to 30 Degrees," Journal of Spacecraft and Rockets, vol. 24, pp. 179-181, 1987.

[8] L. Hee-Chang and L. Sang-Joon, "Flow Control of Circular Cylinders with Longitudinal Grooved Surfaces," AIAA Journal, vol. 40, pp. 2027-36, 2002.

[9] R. A. Wallis, "Wind Tunnel Studies of Leading Edge Separation Phenomena on a Quarter Scale Model of the Outer Panel of the Handley Page Victor Wing with and without Nose Droop," $R$ \& $M$ 3455, 1965.

[10] J. J. Bertin, Aerodynamics for Engineers, 4th ed., Prentice Hall, pp.134-136, 2002.

[11] S. M. Gottlieb, "Two-Dimensional Wind-Tunnel Investigation of Two NACA 6-Series Airfoils with Leading-Edge Slats," NACA RM No. L8K22, Langley Aeronautical Laboratory Langley Field, VA, Jan 1949.

[12] F. E. Weick and R. Sanders, "Wind-Tunnel Tests on Combinations of a Wing With Fixed Auxiliary Airfoils Having Various Chords and Profiles," NACA Tech Report No. 472, Langley Research Center, VA, 1934.

[13] C. J. Wenzinger and F. M. Rogallo, "Resume of Air-load Data on Slats and Flaps," NACA TN-720, Langley Research Center, VA, 1939.

[14] J. C. Lin, "Control of Turbulent Boundary-Layer Separation using Micro-Vortex Generators," AIAA-1999-3404, presented at the 30th AIAA Fluid Dynamics Conference, Norfolk, VA, 1999. 
[15] B. Gardarin, et al., "Flow Separation Control with Vortex Generators," AIAA-2008-3773, in 4th AIAA Flow Control Conference, June 23-26, 2008, Seattle, WA, 2008.

[16] M. Kerho and B. Kramer, "Enhanced Airfoil Design Incorporating Boundary Layer Mixing Devices," AIAA-2003-211, presented at the 41st AIAA Aerospace Sciences Meeting \& Exhibit Jan 6-9 2003, Reno, NV, 2003.

[17] M. P. Patel and T. S. e. a. Prince, "Control of Aircraft Stall via Embedded Pressure Sensors and Deployable Flow Effectors," AIAA-2002-3170, presented at the 1st Flow Control Conference, Jun 24-26 2002, St. Louis, Missouri, 2002.

[18] D. K. Nowak and U. P. Solies, "Wind-Tunnel Tests of a High Lift Generation and Stall/Spin Recovery System," Journal of Aircraft, vol. 37, pp. 383-389, 2000.

[19] T. C. Corke, et al., "Modification in Drag of Turbulent Boundary Layers Resulting from Manipulation of Large-Scale Structures," NASA Contractor Reports 81A26501, 1981.

[20] G. Iuso and M. Onorato, "Turbulent Boundary Layer Manipulation by Outer-layer Devices," Meccanica, vol. 30, pp. 359-76, 1995.

[21] J. B. Anders and R. D. Watson, "Airfoil Large-Eddy Breakup Devices for Turbulent Drag Reduction," in AIAA-1985-520, Shear Flow Control Conference, Mar 12-14, 1985, Boulder, CO, 1985.

[22] J. B. Anders, "Large Eddy Breakup Devices as Low Reynolds Number Airfoils," in $S A E$ Technical paper series 861769, Aerospace Technology Conference and Exposition., Long Beach, CA, 1986.

[23] J. B. Anders, "Outer-layer Manipulators for Turbulent Drag Reduction," NASA Contractor Report 91A12695, Langley Research Center, 1990.

[24] Simpson A., et al., "Aeromechanics of Inflatable Airfoils," AIAA-2004-2233, presented at the 34th AIAA Fluid Dynamics Conference and Exhibit, Jun 28-1, Portland, Oregon, 2004.

[25] McMasters J. H. and M. L. Henderson, "Low-speed Single-element Airfoil Synthesis," NASA Langley Research Center, The Science and Technology of Low Speed and Motorless Flight, Accession No. 79N23890, Pt. 1; p 1-31, 1979.

[26] ANSYS Inc. (2009). ANSYS FLUENT 12.0 Theory Guide. Available: file://C:/PROGRA 1/ANSYSI 1/v120/commonfiles/help/en-us/FLUENT/pdf/th/flth.pdf [Cited on 4/20/2011]

[27] P. R. Spalart, Allmaras, S., "A One-equation Turbulence Model for Aerodynamic Fows," AIAA-92-0439, 1992.

[28] D. C. Wilcox, Turbulence Modeling for CFD. Canada: Industries, Inc., pp. 103-218, 1998. 
[29] P. R. Spalart, et al., "A New Version of Detached-eddy Simulation, Resistant to Ambiguous Grid Densities," Theoretical and Computational Fluid Dynamics, vol. 20, pp. 181-95, 2006.

[30] P. R. Spalart, "Young Person's Guide to Detached-eddy Simulation Grids," NASA/CR-2001211032, Prepared for Langley Research Center under Contract NAS1-97040, 2001.

[31] A. Probst, et al., "A Comparison of Detached-eddy Simulation and Reynolds-stress Modelling Applied to the Flow over a Backward-facing Step and an Airfoil at Stall," in AIAA-2010-920, 48th AIAA Aerospace Sciences Meeting, January 4-7, 2010, Orlando, FL, 2010.

[32] F. R. Menter and M. Kuntz, "Adaptation of Eddy-viscosity Turbulence Models to Unsteady Separated Flow Behind Vehicles," presented at the Symposium on the aerodynamics of heavy vehicles: trucks, buses and trains, Monterey, 2002, Spriner, Berlin, pp. 339-352, 2004.

[33] K. A. Hoffmann and S. T. Chiang, Computational Fluid Dynamics, 4th ed., vol. 1, Wichita, KS, EESbooks, pp. 358-420, 2000.

[34] J. Wild, "Validation of Numerical Optimization of High-Lift Multi-Element Airfoils based on Navier-Stokes-Equations," AIAA-2002-2939, presented at the 20th AIAA Applied Aerodynamics Conference, St. Louis, Missouri, Jun 24-26, 2002.

[35] A. Hay and M. Visonneau, "Local Mesh Adaptation and Remeshing Technique Applied to a Multiple Element Airfoil," AIAA-2005-5337, presented at the 17th AIAA CFD conference Toronto, Canada, 2005.

[36] M. S. Genc, et al., "An Experimental and Computational Study of Low Re Number Transitional Flows over an Aerofoil with Leading Edge Slat," AIAA-2008-8877, in 8th AIAA Aviation Technology, Integration and Operations (ATIO) Conference, September 14-19, 2008, Anchorage, AK, 2008.

[37] I. H. Abbott and A. E. Von Doenhoff, Theory of Wing Sections Including a Summary of Airfoil Data. New York, Dover, pp. 570-571, 1959.

[38] A. E. Von Doenhoff and F. T. J. Abbott, "The Langley Two-dimensional Low-turbulence Pressure Tunnel," NACA TN No. 1283, Langley Memorial Aeronautical Laboratory, Langley Field, VA, 1947.

[39] D. C. Montgomery, Design and Analysis of Experiments, 4th ed., John Wiley \& Sons Australia, pp. 175-210, 1999. 